\title{
Half-integral weight modular forms and application to neutrino mass models
}

\author{
Xiang-Gan Liu, ${ }^{1,2, *}$ Chang-Yuan Yao $\oplus^{3, \dagger}$ Bu-Yao Qu, ${ }^{1,2, \$}$ and Gui-Jun Ding ${ }^{1,2, \S}$ \\ ${ }^{1}$ Peng Huanwu Center for Fundamental Theory, Hefei, Anhui 230026, China \\ ${ }^{2}$ Interdisciplinary Center for Theoretical Study and Department of Modern Physics, \\ University of Science and Technology of China, Hefei, Anhui 230026, China \\ ${ }^{3}$ School of Physics, Nankai University, Tianjin 300071, China
}

(Received 25 September 2020; accepted 9 December 2020; published 30 December 2020)

\begin{abstract}
We generalize the modular invariance approach to include the half-integral weight modular forms. Accordingly the modular group should be extended to its metaplectic covering group for consistency. We introduce the well-defined half-integral weight modular forms for congruence subgroup $\Gamma(4 N)$ and show that they can be decomposed into the irreducible multiplets of finite metaplectic group $\tilde{\Gamma}_{4 N}$. We construct concrete expressions of the half-integral/integral modular forms for $\Gamma(4)$ up to weight 6 and arrange them into the irreducible multiplets of $\tilde{\Gamma}_{4}$. We present three typical models with $\tilde{\Gamma}_{4}$ modular symmetry for neutrino masses and mixing, and the phenomenological predictions of each model are analyzed numerically.
\end{abstract}

DOI: 10.1103/PhysRevD.102.115035

\section{INTRODUCTION}

How to understand the mass hierarchies and flavor mixing patterns of quark and lepton is still one of the greatest challenges in particle physics. The origin of the large mass hierarchies among quarks and charged leptons, the tiny but nonzero neutrino masses, and observed drastically different patterns of quark and lepton flavor mixing cannot be explained by the Standard Model (SM). In many scenarios beyond the SM, flavor symmetry is still a very interesting and promising approach to solve these mysteries. Especially in recent years, the attempt to explain the large mixing angles in lepton sector with some discrete non-Abelian flavor groups has made good progress. However, generally a large number of scalar fields socalled flavons transforming nontrivially under discrete flavor symmetry are necessary to spontaneously break the flavor symmetry group. Moreover, auxiliary symmetries such as the product of cyclic groups are generally introduced to forbid the dangerous terms and to achieve the desired vacuum alignment in the neutrino and charged

\footnotetext{
hepliuxg@mail.ustc.edu.cn

†yaocy@nankai.edu.cn

*qubuyao@mail.ustc.edu.cn

dinggj@ustc.edu.cn
}

Published by the American Physical Society under the terms of the Creative Commons Attribution 4.0 International license. Further distribution of this work must maintain attribution to the author(s) and the published article's title, journal citation, and DOI. Funded by SCOAP. lepton sectors. In short, the flavor symmetry breaking sector has to be intelligently designed and the structure is complex in traditional discrete flavor symmetry models.

Recently modular invariance has been suggested as the origin of flavor symmetry [1]. Notice that modular symmetry naturally appears in torus and orbifold compactifications of string theory. Some recent related work about the modular symmetry on $T^{2}$ and $T^{2} \times T^{2}$ with magnetic fluxes can been seen in $[2,3]$, where zero-modes wave functions behave as weight $1 / 2$ and 1 modular forms. The modular invariance as flavor symmetry is a bottom-up approach [1], flavons are not absolutely necessary, and flavor symmetry can be uniquely broken by the vacuum expectation value of complex modulus $\tau$. Therefore, the above mentioned issue of vacuum alignment is drastically simplified although a moduli stabilization mechanism is needed. In this approach, the Yukawa couplings are modular forms which are holomorphic functions of modulus $\tau$, and the superpotential is completely determined by modular invariance in the limit of supersymmetry while the Kähler potential is not fixed by modular symmetry [4]. In a top-down approach motivated from string theory [5-7], the modular and traditional flavor symmetries are combined to form the eclectic flavor groups. The Kähler potential as well as the representation and weight assignment for the matter fields are severely restricted in this scheme although the order of eclectic flavor group is larger.

The finite modular group $\Gamma_{2} \cong S_{3}$ [8-11], $\Gamma_{3} \cong A_{4}$ $[1,8,9,12-32], \quad \Gamma_{4} \cong S_{4} \quad[25,33-40], \quad \Gamma_{5} \cong A_{5} \quad[38,41,42]$ and $\Gamma_{7} \cong \operatorname{PSL}\left(2, \mathbb{Z}_{7}\right)$ [43] have been considered. The quark masses and CKM parameters together with the 
TABLE I. The dimension formula of $\operatorname{dim} \mathcal{M}_{r}(\Gamma(N))$ for $N=4,5,7$, 9, the linear space $\left.\mathcal{M}_{r}(\Gamma(N))\right|_{k=1}$ of the lowest fractional weight modular forms, and the finite metaplectic group $\tilde{\Gamma}_{N}$. Notice that the functions $f_{n}^{(N)}(\tau)$ and the theta constants $\theta_{2,3}(0 \mid 2 \tau)$ are defined in Eqs. (32) and (37) respectively.

\begin{tabular}{ccccccc}
\hline \hline$N$ & Weight $r$ & \multicolumn{1}{c}{$\operatorname{dim} \mathcal{M}_{r}(\Gamma(N))$} & $\left.\mathcal{M}_{r}(\Gamma(N))\right|_{k=1}$ & $\tilde{\Gamma}_{N}$ & $\left|\tilde{\Gamma}_{N}\right|$ & GAP ID \\
\hline 4 & $k / 2$ & $k+1$ & $\left\{\theta_{3}(0 \mid 2 \tau), \theta_{2}(0 \mid 2 \tau)\right\}$ & $\tilde{S}_{4}$ & 96 & {$[96,67]$} \\
5 & $k / 5$ & $k+1$ & $\left\{f_{1}^{(5)}(\tau), f_{3}^{(5)}(\tau)\right\}$ & $\Gamma_{5}^{\prime} \times Z_{5}$ & 600 & {$[600,54]$} \\
7 & $2 k / 7$ & $\begin{cases}4 k-2 & (\text { for } k \geq 2) \\
3 & (\text { for } k=1)\end{cases}$ & $\left\{f_{1}^{(7)}(\tau), f_{3}^{(7)}(\tau), f_{5}^{(7)}(\tau)\right\}$ & $\Gamma_{7} \times Z_{7}$ & 1176 & {$[1176,212]$} \\
9 & $k / 3$ & $\begin{cases}9 k-9 & \text { (for } k \geq 3) \\
10 & (\text { for } k=2) \\
4 & \text { (for } k=1)\end{cases}$ & $\left\{f_{1}^{(9)}(\tau), f_{3}^{(9)}(\tau), f_{5}^{(9)}(\tau), f_{7}^{(9)}(\tau)\right\}$ & $\tilde{\Gamma}_{9}$ & 1944 & {$[1944,2976]$} \\
& & & & & \\
\hline \hline
\end{tabular}

lepton masses and mixing can be explained by using modular symmetry $[15,18,31,44]$. Modular symmetry has been also discussed in $S U(5)$ grand unification theory $[10,14]$. Notably, the dynamics of modular symmetry could be tested at present and future neutrino oscillation experiments [45]. The modular symmetry can be consistently combined with the generalized $C P$ symmetry [46-50]. Multiple modular symmetries with direct product has been proposed [35,37]. A comprehensive discussion about flavor symmetry, $C P$ symmetry and modular invariance in string theory was recently given in $[47,51]$. The modular invariance approach is generalized to include the odd weight modular forms which can be arranged into irreducible representations of the homogeneous finite modular group $\Gamma_{N}^{\prime}$ [52]. $\Gamma_{N}^{\prime}$ is the double covering of the inhomogeneous finite modular group $\Gamma_{N}$. A simultaneous description of quark and lepton sectors can be achieved in the modular symmetries $\Gamma_{3}^{\prime} \cong T^{\prime}[44,52]$ and $\Gamma_{4}^{\prime} \cong S_{4}^{\prime}$ [53,54]. It is notable that quite predictive flavor models can be constructed with $S_{4}^{\prime}$ [53]. The modular symmetry has the merits of both Abelian flavor symmetry and discrete non-Abelian flavor symmetry, it can naturally generates texture zeros in fermion mass matrix [44] after including odd weight modular forms, and the modular weight can play the role of Froggatt-Nielsen charge to generate the fermion mass hierarchies [30].

In this work, we further extend the modular invariance approach to include half-integral weight modular forms. In order to consistently discuss the action of the modular transformations of the half-integral weight modular forms, one should consider the metaplectic covering of the classical modular group $\mathrm{SL}_{2}(\mathbb{Z})$. Accordingly the framework of modular invariance is extended to the metaplectic modular invariance. The more general modular forms of rational weights can be studied in a similar way. It is known that the half-integral weight modular forms of congruence subgroup can (and can only) be defined at level $4 N$. We find that the half-integral weight modular forms for $\Gamma(4 N)$ can be arranged into irreducible multiplets of the finite metaplectic modular group $\tilde{\Gamma}_{4 N}$ which is the quadruple covering of the inhomogeneous finite modular group $\Gamma_{4 N}$ or the double covering of the homogeneous finite modular group $\Gamma_{4 N}^{\prime}$. In this work, we focus on the lowest level case of $\Gamma(4)$, and use the corresponding modular forms of half-integral weight to construct lepton mass models.

The rest of the paper is organized as follows. In Sec. II, we introduce the metaplectic group and give the definition of the half-integral weight modular forms. We show that the half-integral weight modular forms of $\Gamma(4 N)$ arrange themselves into different irreducible multiplets of the finite metaplectic group $\tilde{\Gamma}_{4 N}$. We also present some useful known results of rational weight modular forms by mathematician, the explicit expressions of the lowest rational weight modular forms for certain congruence subgroup $\Gamma(N)$, and the corresponding finite metaplectic group $\tilde{\Gamma}_{N}$ are summarized in Table I. In Sec. III, we construct the halfintegral and integral weight modular forms for $\Gamma(4)$ up to weight 6 in terms of Jacobi theta constants, and organize them into irreducible representations of finite metaplectic group $\tilde{\Gamma}_{4}=\tilde{S}_{4}$. In Sec. IV, we generalize the modular invariant theory to include the half-integral weight modular forms. Moreover, we present three phenomenologically viable models for lepton masses and flavor mixing based on the finite metaplectic group $\tilde{\Gamma}_{4} \equiv \tilde{S}_{4}$. The multiplier systems of the rational weight modular forms are given in Appendix A. The group theory of $\tilde{S}_{4}$ and the ClebschGordan (CG) coefficients in our working basis are presented in Appendix B.

\section{MODULAR SYMMETRY, METAPLECTIC GROUP, AND HALF-INTEGRAL WEIGHT MODULAR FORMS}

The full modular group $\mathrm{SL}_{2}(\mathbb{Z})$ is the group of $2 \times 2$ matrices with integer entries and determinant 1 :

$\mathrm{SL}_{2}(\mathbb{Z})=\left\{\left(\begin{array}{ll}a & b \\ c & d\end{array}\right) \mid a, b, c, d \in \mathbb{Z}, a d-b c=1\right\}$.

It is quite common to use the notation $\Gamma$ for $\mathrm{SL}_{2}(\mathbb{Z})$. It is well-known that $\mathrm{SL}_{2}(\mathbb{Z})$ is finitely generated, and its generators are usually chosen to be $S$ and $T$, 


$$
S=\left(\begin{array}{cc}
0 & 1 \\
-1 & 0
\end{array}\right), \quad T=\left(\begin{array}{ll}
1 & 1 \\
0 & 1
\end{array}\right)
$$

which satisfy the relations

$$
S^{4}=(S T)^{3}=I .
$$

Here $I$ is the two dimensional unit matrix. Let $N$ be a positive integer, the principal congruence subgroup $\Gamma(N)$ of level $\mathrm{N}$ is defined as

$$
\begin{gathered}
\Gamma(N)=\left\{\left(\begin{array}{ll}
a & b \\
c & d
\end{array}\right) \in \mathrm{SL}_{2}(\mathbb{Z}) \mid a, d=1(\bmod N),\right. \\
b, c=0(\bmod N)\},
\end{gathered}
$$

which implies that $\Gamma(N)$ is a normal subgroup of finite index in $\mathrm{SL}_{2}(\mathbb{Z})$, and obviously we have $\Gamma(1)=\mathrm{SL}_{2}(\mathbb{Z})$. We denote by $\mathcal{H}$ the upper half plane, i.e., the set of complex numbers $\tau$ with $\operatorname{Im}(\tau)>0$. We can view elements of $\mathrm{SL}_{2}(\mathbb{Z})$ as acting in the following way on $\mathcal{H}$ :

$\gamma=\left(\begin{array}{ll}a & b \\ c & d\end{array}\right) \in \mathrm{SL}_{2}(\mathbb{Z}), \quad \gamma \tau=\gamma(\tau)=\frac{a \tau+b}{c \tau+d}$.

The modular form $f(\tau)$ of weight $k$ and level $N$ is a holomorphic function of the complex modulus $\tau$ and it satisfies the transformation formula

$$
\begin{aligned}
f\left(\frac{a \tau+b}{c \tau+d}\right) & =(c \tau+d)^{k} f(\tau) \quad \text { for all } \\
\left(\begin{array}{ll}
a & b \\
c & d
\end{array}\right) & \in \Gamma(N) \quad \text { and } \quad \tau \in \mathcal{H} .
\end{aligned}
$$

It has been shown that the modular forms of integral weight $k$ and level $N$ can be arranged into different irreducible representations of the homogeneous finite modular group $\Gamma_{N}^{\prime} \equiv \Gamma / \Gamma(N)$ up to the factor $(c \tau+d)^{k}$ in [52]. In the present work, we intend to include half-integral weight modular forms such that the square root of the $c \tau+d$ appears in the transformation formula. It is crucial to deal with the two branches for the square root in a systematic way. The most common choice and the one we will always use is to choose the principal branch of the square root, i.e., for a complex number $z, z^{1 / 2}$ always means $-\pi / 2<\arg \left(z^{1 / 2}\right) \leq \pi / 2$, in particular if $z<0$ is real, $z^{1 / 2}$ is a pure positive imaginary number such as $(-1)^{1 / 2}=i$. Therefore, $\left(z_{1} z_{2}\right)^{1 / 2}$ is equal to $z_{1}^{1 / 2} z_{2}^{1 / 2}$ only up to a sign \pm 1 , i.e., $\left(z_{1} z_{2}\right)^{1 / 2}=z_{1}^{1 / 2} z_{2}^{1 / 2}$ for $-\pi<\arg \left(z_{1}\right)+\arg \left(z_{2}\right) \leq \pi$ and $\left(z_{1} z_{2}\right)^{1 / 2}=-z_{1}^{1 / 2} z_{2}^{1 / 2}$ otherwise. For an (even or odd) integer $k, z^{k / 2}$ always refer to $\left(z^{1 / 2}\right)^{k}$. Note that this is not always equal to $\left(z^{k}\right)^{1 / 2}$ for $k$ odd. It is nontrivial to define the half-integral weight modular forms, and $J_{k / 2}(\gamma, \tau) \equiv$ $(c \tau+d)^{k / 2}$ is not the automorphy factor anymore, and certain multiplier is generally needed. For instance, the half-integral $k / 2$ weight modular form $f(\tau)$ can be consistently defined for the principal congruence subgroup $\Gamma(4 N)$, it is a holomorphic function of $\tau$ and satisfies the following condition,

$$
\begin{aligned}
f(h \tau) & =v^{k}(h)(c \tau+d)^{k / 2} f(\tau)=v^{k}(h) J_{k / 2}(h, \tau) f(\tau), \\
h & =\left(\begin{array}{ll}
a & b \\
c & d
\end{array}\right) \in \Gamma(4 N),
\end{aligned}
$$

where $v(h)=\left(\frac{c}{d}\right)$ is the Kronecker symbol, it is 1 or -1 here and more details can be found in the textbook [55]. Notice that $v^{k}(h)$ and $J_{k / 2}(h, \tau)$ satisfy the following identities [55,56],

$$
\begin{gathered}
J_{k / 2}\left(\gamma_{1} \gamma_{2}, \tau\right)=\zeta_{k / 2}^{-1}\left(\gamma_{1}, \gamma_{2}\right) J_{k / 2}\left(\gamma_{1}, \gamma_{2} \tau\right) J_{k / 2}\left(\gamma_{2}, \tau\right), \\
\gamma_{1,2} \in \Gamma, \\
v^{k}\left(h_{1} h_{2}\right)=\zeta_{k / 2}\left(h_{1}, h_{2}\right) v^{k}\left(h_{1}\right) v^{k}\left(h_{2}\right), \quad h_{1,2} \in \Gamma(4 N),
\end{gathered}
$$

where $\zeta_{k / 2}\left(\gamma_{1}, \gamma_{2}\right)=\zeta_{1 / 2}^{k}\left(\gamma_{1}, \gamma_{2}\right) \in\left\{1, e^{\pi i k}\right\}$, and $\zeta_{1 / 2}\left(\gamma_{1}, \gamma_{2}\right)$ is the so-called two-cocycle on $\mathrm{SL}_{2}(\mathbb{Z})[55,56]$. Note that $\zeta_{1 / 2}\left(\gamma_{1}, \gamma_{2}\right)$ can only take values +1 and -1 such that $\zeta_{k / 2}\left(\gamma_{1}, \gamma_{2}\right)$ is always equal to 1 for any values of $\gamma_{1}$ and $\gamma_{2}$ if $k$ is even. We denote the factor $\tilde{J}_{k / 2}(h, \tau) \equiv$ $v^{k}(h)(c \tau+d)^{k / 2}$, using Eqs. (8a) and (8b) it is easy to check $\tilde{J}_{k / 2}$ satisfies the cocycle relation

$\tilde{J}_{k / 2}\left(h_{1} h_{2}, \tau\right)=\tilde{J}_{k / 2}\left(h_{1}, h_{2} \tau\right) \tilde{J}_{k / 2}\left(h_{2}, \tau\right), \quad h_{1,2} \in \Gamma(4 N)$.

This means that $\tilde{J}_{k / 2}(h, \tau)$ is the correct automorphy factor for $\Gamma(4 N)$, this generalized automorphy factor eliminates the ambiguity caused by half-integral weight, and the halfintegral weight modular form defined in Eq. (7) really makes sense.

\section{A. Metaplectic group}

In order to discuss the action of the full modular group on the half-integral modular forms, one has to consider the metaplectic (twofold) cover group $\mathrm{Mp}_{2}(\mathbb{Z})$ of $\mathrm{SL}_{2}(\mathbb{Z})$ [57]. For notational simplicity, we shall denote $\mathrm{Mp}_{2}(\mathbb{Z})$ as $\tilde{\Gamma}$ in the following. The elements of $\tilde{\Gamma}$ can be written in the form [57]: 
$\tilde{\Gamma}=\left\{\tilde{\gamma}=(\gamma, \phi(\gamma, \tau)) \mid \gamma=\left(\begin{array}{ll}a & b \\ c & d\end{array}\right) \in \Gamma, \phi(\gamma, \tau)^{2}=(c \tau+d)\right\}$,

which implies $\phi(\gamma, \tau)= \pm(c \tau+d)^{1 / 2}=\epsilon J_{1 / 2}(\gamma, \tau)$ with $\epsilon= \pm 1$. The multiplication law of $\operatorname{Mp}_{2}(\mathbb{Z})$ is given by

$\left(\gamma_{1}, \phi\left(\gamma_{1}, \tau\right)\right)\left(\gamma_{2}, \phi\left(\gamma_{2}, \tau\right)\right)=\left(\gamma_{1} \gamma_{2}, \phi\left(\gamma_{1}, \gamma_{2} \tau\right) \phi\left(\gamma_{2}, \tau\right)\right)$,

or equivalently

$$
\begin{aligned}
& \left(\gamma_{1}, \epsilon_{1} J_{1 / 2}\left(\gamma_{1}, \tau\right)\right)\left(\gamma_{2}, \epsilon_{2} J_{1 / 2}\left(\gamma_{2}, \tau\right)\right) \\
& \quad=\left(\gamma_{1} \gamma_{2}, \epsilon_{1} \epsilon_{2} \zeta_{1 / 2}\left(\gamma_{1}, \gamma_{2}\right) J_{1 / 2}\left(\gamma_{1} \gamma_{2}, \tau\right)\right),
\end{aligned}
$$

where $\epsilon_{1}, \epsilon_{2} \in\{ \pm 1\}$. Obviously each element $\gamma \in \Gamma$ corresponds to two elements $\tilde{\gamma}=\left(\gamma, \pm J_{1 / 2}(\gamma, \tau)\right)$ of the metaplectic group $\tilde{\Gamma}$. Let us consider the natural projection mapping $P:\left(\gamma, \pm J_{1 / 2}(\gamma, \tau)\right) \mapsto \gamma$, then it is easy to see the kernel $\operatorname{Ker}(P)=(1, \pm 1) \cong\{ \pm 1\}$, therefore, $\tilde{\Gamma}$ can be viewed as the central extension of the modular group $\Gamma$ by the group $\{ \pm 1\}$.

Using the generators $S$ and $T$ of $\mathrm{SL}_{2}(\mathbb{Z})$, it is easy to see that the metaplectic group $\tilde{\Gamma}$ can be generated by $\tilde{S}$ and $\tilde{T}[56,58]$ :

$$
\tilde{S}=\left(\left(\begin{array}{cc}
0 & 1 \\
-1 & 0
\end{array}\right),-\sqrt{-\tau}\right), \quad \tilde{T}=\left(\left(\begin{array}{ll}
1 & 1 \\
0 & 1
\end{array}\right), 1\right),
$$

where $\sqrt{-\tau}$ denotes the principal branch of the square root, possessing positive real part. Notice that $\tilde{S}$ is of order 8 while $\tilde{T}$ is of infinite order, and we have

$$
\begin{gathered}
\tilde{S} \tilde{T}=\left(\left(\begin{array}{cc}
0 & 1 \\
-1 & -1
\end{array}\right),-\sqrt{-\tau-1}\right), \\
\tilde{S}^{2} \equiv \tilde{R}=\left(\left(\begin{array}{cc}
-1 & 0 \\
0 & -1
\end{array}\right),-i\right),
\end{gathered}
$$

which are of orders 3 and 4, respectively. Hence, the generators $\tilde{S}$ and $\tilde{T}$ fulfill the relations

$$
\tilde{S}^{8}=(\tilde{S} \tilde{T})^{3}=1,
$$

or equivalently

$$
\tilde{S}^{2}=\tilde{R}, \quad(\tilde{S} \tilde{T})^{3}=\tilde{R}^{4}=1, \quad \tilde{T} \tilde{R}=\tilde{R} \tilde{T},
$$

Because $\tilde{R}$ is commutable with both generators $\tilde{S}$ and $\tilde{T}, \tilde{R}$ generates the center of $\tilde{\Gamma}$. Notice the identities $\tilde{R}^{2}=\left(\left(\begin{array}{l}1 \\ 0\end{array}\right),-1\right)$ and $\tilde{R}^{2}\left(\gamma, J_{1 / 2}(\gamma, \tau)\right)=\left(\gamma,-J_{1 / 2}(\gamma, \tau)\right)$, therefore, the modular group $\mathrm{SL}_{2}(\mathbb{Z})$ is isomorphic to the quotient of $\operatorname{Mp}_{2}(\mathbb{Z})$ over the $Z_{2}$ subgroup $Z_{2}^{\tilde{R}^{2}}=\left\{1, \tilde{R}^{2}\right\}$,

$$
\mathrm{Mp}_{2}(\mathbb{Z}) / Z_{2}^{\tilde{R}^{2}} \cong \mathrm{SL}_{2}(\mathbb{Z})
$$

A well-known metaplectic congruence subgroup is $[56,58]$ :

$$
\tilde{\Gamma}(4 N)=\left\{\tilde{h}=\left(h, v(h) J_{1 / 2}(h, \tau)\right) \mid h \in \Gamma(4 N)\right\},
$$

where $v(h)=\left(\frac{c}{d}\right)$ is the Kronecker symbol. $\tilde{\Gamma}(4 N)$ is an infinite normal subgroup of $\tilde{\Gamma}$ and it is isomorphic to the principal congruence subgroup $\Gamma(4 N)$. Likewise the finite metaplectic group is the quotient group $\tilde{\Gamma}_{4 N} \equiv \tilde{\Gamma} / \tilde{\Gamma}(4 N)$. It is easy to check

$$
\tilde{T}^{4 N}=\left(\left(\begin{array}{cc}
1 & 4 N \\
0 & 1
\end{array}\right), 1\right) \in \tilde{\Gamma}(4 N) .
$$

Consequently, the relation

$$
\tilde{T}^{4 N}=1
$$

is generally fulfilled in the group $\tilde{\Gamma}_{4 N}$. In the present work, we focus on the lowest case $N=1$. The finite metaplectic group $\tilde{\Gamma}_{4}$ denoted as $\tilde{S}_{4}$ is a group of order 96 with group ID $[96,67]$ in GAP [59]. The conjugacy classes and the irreducible representations of $\tilde{S}_{4}$ are given in Appendix B. For larger $N$, the relations in Eqs. (15) and (20) or equivalently Eqs. (16) and (20) are not sufficient and addition relations are needed to render the group $\tilde{\Gamma}_{4 N}$ finite. For instance, for the case of $N=2$, the multiplication rules of $\tilde{\Gamma}_{8}$ for the generators $\tilde{S}, \tilde{T}$ and $\tilde{R}$ are ${ }^{1}$

$$
\begin{aligned}
\tilde{S}^{2} & =\tilde{R}, \quad(\tilde{S} \tilde{T})^{3}=\tilde{R}^{4}=\tilde{T}^{8}=\tilde{R}^{2} \tilde{S} \tilde{T}^{6} \tilde{S} \tilde{T}^{4} \tilde{S} \tilde{T}^{2} \tilde{S} \tilde{T}^{4}=1, \\
\tilde{T} \tilde{R} & =\tilde{R} \tilde{T} .
\end{aligned}
$$

Thus $\tilde{\Gamma}_{8}$ is a group of order 768 and its group ID in GAP is [768,1085324].

\section{B. Half-integral weight modular forms}

For an element $\tilde{\gamma}=(\gamma, \phi(\gamma, \tau))$, we define the weight$k / 2$ slash operator $\mid[\tilde{\gamma}]_{k / 2}$ on the modular function $f(\tau)$ as [57]:

$$
f(\tau) \mid[\tilde{\gamma}]_{k / 2}=f(\gamma \tau) \phi^{-k}(\gamma, \tau) .
$$

The slash operator has the property,

$$
f(\tau)\left|\left[\tilde{\gamma}_{1}\right]_{k / 2}\right|\left[\tilde{\gamma}_{2}\right]_{k / 2}=f(\tau) \mid\left[\tilde{\gamma}_{1} \tilde{\gamma}_{2}\right]_{k / 2}, \tilde{\gamma}_{1,2} \in \tilde{\Gamma} .
$$

\footnotetext{
${ }^{1}$ The relations can also be written as $\tilde{S}^{8}=(\tilde{S} \tilde{T})^{3}=\tilde{T}^{8}=$ $\tilde{S}^{5} \tilde{T}^{6} \tilde{S} \tilde{T}^{4} \tilde{S} \tilde{T}^{2} \tilde{S} \tilde{T}^{4}=1, \tilde{T} \tilde{S}^{2}=\tilde{S}^{2} \tilde{T}$.
} 
The modular forms of the metaplectic congruence subgroup $\tilde{\Gamma}(4 N)$ is a holomorphic modular function invariant under the action of the slash operator $\mid[\tilde{h}]_{k / 2}$, i.e.,

$$
\begin{aligned}
f(\tau) \mid[\tilde{h}]_{k / 2} & =f(\tau) \quad \text { or } \quad f(h \tau)=\phi^{k}(h, \tau) f(\tau), \\
\tilde{h} & \in \tilde{\Gamma}(4 N) .
\end{aligned}
$$

This is actually the same as the condition in Eq. (7) which the half-integral weight modular forms of $\Gamma(4 N)$ should satisfy. It can be seen that $\tilde{\Gamma}(4 N)$ is the more natural group acting on the half-integral weight modular forms of $\Gamma(4 N)$.

The weight $k / 2$ modular forms of $\tilde{\Gamma}(4 N)$ span a linear space $\mathcal{M}_{k / 2}(\tilde{\Gamma}(4 N))$ of finite dimension $n=$ $\operatorname{dim} \mathcal{M}_{k / 2}(\tilde{\Gamma}(4 N))$. Let us denote a multiplet of linearly independent modular form $f(\tau) \equiv\left(f_{1}(\tau), f_{2}(\tau), \ldots, f_{n}(\tau)\right)^{T}$, $\tilde{\gamma}=\left(\gamma, \epsilon J_{1 / 2}(\gamma, \tau)\right)$ and $\tilde{h}=\left(h, v(h) J_{1 / 2}(h, \tau)\right)$ stand for a generic element of $\tilde{\Gamma}$ and $\tilde{\Gamma}(4 N)$ respectively. It is straightforward to check that the following identity is fulfilled,

$$
f(\tau)\left|[\tilde{\gamma}]_{k / 2}\right|[\tilde{h}]_{k / 2}=f(\tau) \mid[\tilde{\gamma}]_{k / 2} .
$$

This means that the function $f(\tau) \mid[\tilde{\gamma}]_{k / 2}$ is invariant under the action of the slash operator $\mid[\tilde{h}]_{k / 2}$. In other words, $f(\tau) \mid[\tilde{\gamma}]_{k / 2}=f(\gamma \tau) \phi^{-k}(\gamma, \tau)$ should be a modular form of $\tilde{\Gamma}(4 N)$, therefore, $f(\tau) \mid[\tilde{\gamma}]_{k / 2}$ can be written as a linear combination of $f_{i}(\tau)$ :

$$
f(\tau) \mid[\tilde{\gamma}]_{k / 2}=\rho(\tilde{\gamma}) f(\tau) \quad \text { or } \quad f(\gamma \tau)=\phi^{k}(\gamma, \tau) \rho(\tilde{\gamma}) f(\tau),
$$

where $\rho(\tilde{\gamma})$ is a $n \times n$ dimensional matrix depending on $\tilde{\gamma}$. Using the identity $f(\tau)\left|\left[\tilde{\gamma}_{1}\right]_{k / 2}\left[\tilde{\gamma}_{2}\right]_{k / 2}=f(\tau)\right|\left[\tilde{\gamma}_{1} \tilde{\gamma}_{2}\right]_{k / 2}$ in Eq. (23), we can obtain ${ }^{2}$

$$
\rho\left(\tilde{\gamma}_{1}\right) \rho\left(\tilde{\gamma}_{2}\right)=\rho\left(\tilde{\gamma}_{1} \tilde{\gamma}_{2}\right) .
$$

Hence, $\rho$ is a linear representation of the metaplectic group $\tilde{\Gamma}$. For $\quad \tilde{\gamma}=\tilde{h}=\left(h, v(h) J_{1 / 2}(h, \tau)\right) \in \tilde{\Gamma}(4 N)$, Eq. (26) gives us

$$
f(\tau) \mid[\tilde{h}]_{k / 2}=\rho(\tilde{h}) f(\tau) .
$$

Comparing with the definition of modular form $f(\tau) \mid[\tilde{h}]_{k / 2}=f(\tau)$ in Eq. (24), we obtain $\rho(\tilde{h})=1$. As a consequence, $\rho(\tilde{\gamma})$ is actually a linear representation of the quotient group $\tilde{\Gamma}_{4 N} \equiv \tilde{\Gamma} / \tilde{\Gamma}(4 N)$. The finite representation $\rho$ can always be decomposed into a direct sum of irreducible unitary representations of $\tilde{\Gamma}_{4 N}$ such that the modular forms of half-integral weights can be arranged into different irreducible representations of the finite group $\tilde{\Gamma}_{4 N}$. Furthermore, applying Eq. (26) for $\tilde{\gamma}=\tilde{R}$, we obtain

$$
f(\tilde{R} \tau)=f(\tau)=(-i)^{k} \rho(\tilde{R}) f(\tau),
$$

which implies

$$
\rho(\tilde{R})=i^{k}, \begin{cases}k \text { odd: } & \rho^{4}(\tilde{R})=1 \\ k \text { even: } & \rho^{2}(\tilde{R})=1 .\end{cases}
$$

Taking into account with the general relations in Eqs. (15) and (20), we can know

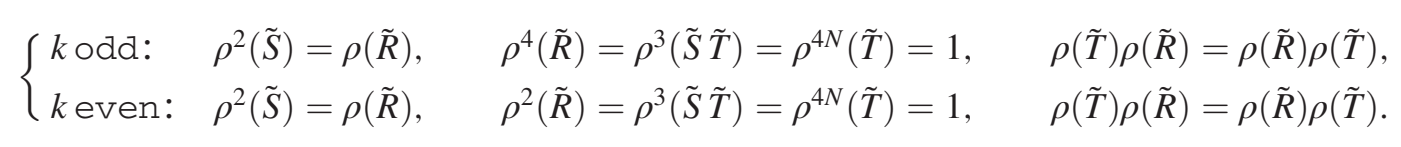

Notice that the representation matrices of the generators $\tilde{S}$ and $\tilde{T}$ satisfy the same relations as those of the homogeneous finite modular group $\Gamma_{4 N}^{\prime}$ [52]. The equations in Eq. (31) show explicitly that the half-integral weight modular form can be decomposed into irreducible representation of finite metaplectic group $\tilde{\Gamma}_{4 N}$, and the integral weight modular forms are arranged into irreducible multiplets of $\Gamma_{4 N}^{\prime}$.

\footnotetext{
${ }^{2}$ Analogously we find that $\rho$ forms a projective representation of the modular group $\Gamma: \rho\left(\gamma_{1} \gamma_{2}\right)=\zeta_{k / 2}\left(\gamma_{1}, \gamma_{2}\right) \rho\left(\gamma_{1}\right) \rho\left(\gamma_{2}\right)$ for $\gamma_{1,2} \in \Gamma$. That is to say, the projection representation is lifted to the linear representation by extending the $\Gamma$ to the metaplectic group $\tilde{\Gamma}$.
}

\section{Rational weight modular forms}

Analogously, modular forms of rational weights $r$ can be defined for certain congruence subgroups. Similar to Eq. (7), a multiplier system $v(\gamma)$ is needed such that $v(\gamma)(c \tau+d)^{r}$ is the correct automorphy factor satisfying the cocycle relation, and the ambiguity of multivalued branches caused by the rational power is properly eliminated. It is also a big challenge to explicitly construct the basis of the linear space of the rational weight modular forms. It is remarkable that some mathematicians have found out the multiplier system $v(\gamma)$ for the principal congruence subgroup $\Gamma(N)$ with odd integer $N \geq 5$, the explicit expression of $v(\gamma)$ is given in Appendix A, and the corresponding modular forms of rational weights are constructed [60,61]. We will describe the main results below. 
First of all, for any odd integer $5 \leq N \leq 13$, the ring of the modular forms of rational weight $r=(N-3) /(2 N)$ for the principal congruence subgroup $\Gamma(N)$ can be constructed from the holomorphic functions $f_{n}^{(N)}(\tau)$ [60],

$$
f_{n}^{(N)}(\tau)=\theta_{\left(\frac{n}{2 N}, \frac{1}{2}\right)}(N \tau) / \eta(\tau)^{\frac{3}{N}},
$$

where $n$ are odd integers with $1 \leq n \leq N-2$, and the theta constant with characteristic $\left(m^{\prime}, m^{\prime \prime}\right)$ is defined by

$$
\theta_{\left(m^{\prime}, m^{\prime \prime}\right)}(\tau)=\sum_{m \in \mathbb{Z}} e^{2 \pi i \tau\left(\frac{1}{2}\left(m+m^{\prime}\right)^{2} \tau+\left(m+m^{\prime}\right) m^{\prime \prime}\right)},
$$

and the Dedekind eta function reads

$$
\eta(\tau)=e^{\pi i \tau / 12} \prod_{n=1}^{\infty}\left(1-e^{2 \pi i n \tau}\right) .
$$

Consequently, there are $(N-1) / 2$ linearly independent modular forms $f_{1}^{(N)}, f_{3}^{(N)}, \ldots f_{N-2}^{(N)}$ of rational weight $r=(N-3) /(2 N)$, and the graded rings of modular forms $\mathcal{M}(\Gamma(N))=\bigoplus_{m \geq 1} \mathcal{M}_{m \frac{N-3}{2 N}}(\Gamma(N))$ can be generated by the tensor products of these lowest weight modular forms. The dimension formula of $\mathcal{M}_{m \frac{N-3}{2 N}}(\Gamma(N))$ for any odd integer $N \geq 5$ and any integer $m>\frac{4(N-6)}{N-3}$ is given by $[60,61]$

$$
\begin{aligned}
\operatorname{dim} \mathcal{M}_{m \frac{(N-3)}{2 N}}(\Gamma(N))= & \frac{N^{2}[m(N-3)-2(N-6)]}{48} \\
& \times \prod_{p \mid N}\left(1-\frac{1}{p^{2}}\right),
\end{aligned}
$$

where the product is over the prime divisors $p$ of $N$. As shown in Sec. II B, we expect that rational weight modular forms can be organized into different irreducible multiplets of the finite metaplectic congruence subgroup. We summarize the dimension formula, modular forms of rational weight $r=(N-3) /(2 N)$ and the corresponding finite metaplectic congruence subgroup in Table I. We also include the half-integral weight case which we are concerned with. We would like to mention that the theory of modular forms with real weight and even complex weight are also developed [62], and then the modular group $\mathrm{SL}_{2}(\mathbb{Z})$ should be extended to the universal covering groups. Some concrete examples are given in $[63,64]$.

\section{HALF-INTEGRAL/INTEGRAL WEIGHT MODULAR FORMS OF LEVEL 4}

Half weight modular forms of level $4 N$ have been studied extensively in math since Shimura's original work [65], a general construction of the modular space $\mathcal{M}_{1 / 2}(\Gamma(4 N))$ using theta functions associated with lattices has been given in the literature $[66,67]$. In particular for the simplest case of level $4 N=4$, the linear space of the half weight modular forms can be generated by the following two Jacobi theta constants ${ }^{3}$ :

$$
\mathcal{M}_{1 / 2}(\Gamma(4))=\left\{e_{1}(\tau) \equiv \theta_{3}(0 \mid 2 \tau), e_{2}(\tau) \equiv \theta_{2}(0 \mid 2 \tau)\right\},
$$

with

$$
\begin{aligned}
\theta_{2}(0 \mid 2 \tau) & =\sum_{m \in \mathbb{Z}} e^{2 \pi i \tau\left(m+\frac{1}{2}\right)^{2}}=2 q^{1 / 4}\left(1+q^{2}+q^{6}+q^{12}+\ldots\right), \\
\theta_{3}(0 \mid 2 \tau) & =\sum_{m \in \mathbb{Z}} e^{2 \pi i \tau m^{2}}=1+2 q+2 q^{4}+2 q^{9}+2 q^{16}+\ldots \\
\theta_{4}(0 \mid 2 \tau) & =\sum_{m \in \mathbb{Z}}(-1)^{m} e^{2 \pi i \tau m^{2}} \\
& =1-2 q+2 q^{4}-2 q^{9}+2 q^{16}+\ldots
\end{aligned}
$$

where $q \equiv e^{2 \pi i \tau}$. Using the basic transformation properties of Jacobi theta function [68], we can obtain the following transformation rules under the action of the generators $S$ and $T$,

$\theta_{3}(0 \mid 2 \tau) \stackrel{T}{\rightarrow} \theta_{3}(0 \mid 2 \tau), \quad \theta_{2}(0 \mid 2 \tau) \stackrel{T}{\rightarrow} i \theta_{2}(0 \mid 2 \tau)$,

$\theta_{3}(0 \mid 2 \tau) \stackrel{S}{\rightarrow} \theta_{3}\left(0 \mid-\frac{2}{\tau}\right)=\left(-i \frac{\tau}{2}\right)^{1 / 2} \theta_{3}\left(0 \mid \frac{\tau}{2}\right)$,
$\theta_{2}(0 \mid 2 \tau) \stackrel{S}{\rightarrow} \theta_{2}\left(0 \mid-\frac{2}{\tau}\right)=\left(-i \frac{\tau}{2}\right)^{1 / 2} \theta_{4}\left(0 \mid \frac{\tau}{2}\right)$.

From the definition of the theta constants in Eq. (37), we know

$$
\begin{aligned}
\theta_{3}\left(0 \mid \frac{\tau}{2}\right) & =\sum_{m \in \mathbb{Z}} e^{\pi i\left(\frac{\tau}{2} m^{2}\right)}=\sum_{n \in \mathbb{Z}} e^{2 \pi i \tau n^{2}}+\sum_{n \in \mathbb{Z}} e^{2 \pi i \tau\left(n+\frac{1}{2}\right)^{2}} \\
& =\theta_{3}(0 \mid 2 \tau)+\theta_{2}(0 \mid 2 \tau) \\
\theta_{4}\left(0 \mid \frac{\tau}{2}\right) & =\sum_{m \in \mathbb{Z}}(-1)^{m} e^{\pi i\left(\frac{\tau}{2} m^{2}\right)}=\sum_{n \in \mathbb{Z}} e^{2 \pi i \tau n^{2}}-\sum_{n \in \mathbb{Z}} e^{2 \pi i \tau\left(n+\frac{1}{2}\right)^{2}} \\
& =\theta_{3}(0 \mid 2 \tau)-\theta_{2}(0 \mid 2 \tau) .
\end{aligned}
$$

As a consequence, under the action of $S$ and $T$, the basis vectors $e_{1}(\tau)$ and $e_{2}(\tau)$ transform as

$$
\begin{aligned}
& e_{1}(\tau) \stackrel{T}{\rightarrow} e_{1}(\tau), \quad e_{2}(\tau) \stackrel{T}{\rightarrow} i e_{2}(\tau), \\
& e_{1}(\tau) \stackrel{S}{\rightarrow}\left(-i \frac{\tau}{2}\right)^{1 / 2}\left(e_{1}(\tau)+e_{2}(\tau)\right), \\
& e_{2}(\tau) \stackrel{S}{\rightarrow}\left(-i \frac{\tau}{2}\right)^{1 / 2}\left(e_{1}(\tau)-e_{2}(\tau)\right) .
\end{aligned}
$$

\footnotetext{
${ }^{3}$ These two modular forms also appeared in [54].
} 
We find these two linearly independent modular forms $e_{1}(\tau)$ and $e_{2}(\tau)$ arrange themselves into a doublet denoted as

$$
Y_{\hat{\mathbf{2}}}^{\left(\frac{1}{2}\right)}(\tau)=\left(\begin{array}{c}
e_{1}(\tau) \\
-e_{2}(\tau)
\end{array}\right) \equiv\left(\begin{array}{c}
\vartheta_{1} \\
\vartheta_{2}
\end{array}\right),
$$

which transforms in the two-dimensional irreducible representation $\hat{\mathbf{2}}$ of $\tilde{\Gamma}_{4} \equiv \tilde{S}_{4}$,

$$
\begin{aligned}
& Y_{\hat{\mathbf{2}}}^{\left(\frac{1}{2}\right)}(-1 / \tau)=-\sqrt{-\tau} \rho_{\hat{\mathbf{2}}}(\tilde{S}) Y_{\hat{\mathbf{2}}}^{\left(\frac{1}{2}\right)}(\tau), \\
& Y_{\hat{\mathbf{2}}}^{\left(\frac{1}{2}\right)}(\tau+1)=\rho_{\hat{\mathbf{2}}}(\tilde{T}) Y_{\hat{\mathbf{2}}}^{\left(\frac{1}{2}\right)}(\tau),
\end{aligned}
$$

where the unitary representation matrices $\rho_{\hat{\mathbf{2}}}(\tilde{S})$ and $\rho_{\hat{\mathbf{2}}}(\tilde{T})$ are given in Table VII. All higher (half-integral and integral) weight modular forms can be constructed from the tensor products of $Y_{\hat{2}}^{\left(\frac{1}{2}\right)}(\tau)$ by using the CG coefficients of group $\tilde{S}_{4}$. For instance, we find the weight 1 modular forms make up a triplet $\hat{\mathbf{3}}^{\prime}$ of $\tilde{S}_{4}$,

$$
Y_{\hat{\mathbf{z}}^{\prime}}^{(1)}=\frac{1}{\sqrt{2}}\left(Y_{\hat{\mathbf{2}}}^{\left(\frac{1}{2}\right)} Y_{\hat{\mathbf{2}}}^{\left(\frac{1}{2}\right)}\right)_{\hat{\mathbf{3}}_{\mathbf{s}}^{\prime}}=\left(\begin{array}{c}
\sqrt{2} \vartheta_{1} \vartheta_{2} \\
\vartheta_{1}^{2} \\
-\vartheta_{2}^{2}
\end{array}\right),
$$

where we have multiplied an overall constant $1 / \sqrt{2}$ to make the resulting expression relatively simple. The nontrivial constraint $Y_{1}^{(1) 2}+2 Y_{2}^{(1)} Y_{3}^{(1)}=0$ in Ref. [53] is now trivial. Notice another tensor product $\left(Y_{\hat{2}}^{\left(\frac{1}{2}\right)} Y_{\hat{2}}^{\left(\frac{1}{2}\right)}\right)_{\hat{\mathbf{1}}^{\prime}}=0$ because of the antisymmetric CG coefficient for the contraction $\hat{\mathbf{2}} \otimes \hat{\mathbf{2}} \rightarrow \hat{\mathbf{1}}^{\prime}$. It is straightforward to check that $Y_{\hat{\mathbf{z}}^{\prime}}^{(1)}$ is the same as the original weight one modular forms given in [54] up to a permutation, the discrepancy arises from the different convention for the representation matrices of the generators $\tilde{S}$ and $\tilde{T}$. In a similar fashion, we can obtain higher weight modular forms and decompose them into different irreducible multiplets of $\tilde{S}_{4}$. In the following, we present linearly independent halfintegral and integral weight modular forms up to weight 6 , and we normalize the overall constant for simplicity. There are four linearly independent modular forms of weight $3 / 2$, and they can be arranged into a quartet representation $4^{\prime}$ of $\tilde{S}_{4}$,

$$
Y_{\mathbf{4}^{\prime}}^{\left(\frac{3}{2}\right)}=\frac{1}{\sqrt{3}}\left(Y_{\hat{\mathbf{2}}}^{\left(\frac{1}{2}\right)} Y_{\hat{\mathbf{3}}^{\prime}}^{(1)}\right)_{4^{\prime}}=\left(\begin{array}{c}
\vartheta_{2}^{3} \\
\sqrt{3} \vartheta_{1}^{2} \vartheta_{2} \\
\vartheta_{1}^{3} \\
\sqrt{3} \vartheta_{1} \vartheta_{2}^{2}
\end{array}\right)
$$

The weight 2 modular forms of level 4 can be decomposed into a doublet $\mathbf{2}$ and a triplet $\mathbf{3}$,

$$
\begin{aligned}
& Y_{\mathbf{2}}^{(2)}=\left(Y_{\hat{\mathbf{2}}}^{\left(\frac{1}{2}\right)} Y_{\mathbf{4}^{\prime}}^{\left(\frac{3}{2}\right)}\right)_{\mathbf{2}}=\left(\begin{array}{c}
\vartheta_{1}^{4}+\vartheta_{2}^{4} \\
-2 \sqrt{3} \vartheta_{1}^{2} \vartheta_{2}^{2}
\end{array}\right), \\
& Y_{\mathbf{3}}^{(2)}=\frac{1}{\sqrt{2}}\left(Y_{\hat{\mathbf{2}}}^{\left(\frac{(1)}{2}\right)} Y_{\mathbf{4}^{\prime}}^{\left(\frac{3}{2}\right)}\right)_{\mathbf{3}}=\left(\begin{array}{c}
\vartheta_{1}^{4}-\vartheta_{2}^{4} \\
2 \sqrt{2} \vartheta_{1}^{3} \vartheta_{2} \\
2 \sqrt{2} \vartheta_{1} \vartheta_{2}^{3}
\end{array}\right) .
\end{aligned}
$$

At weight 5/2, we have 6 independent modular forms which transform according to the irreducible representations $\hat{\mathbf{2}}$ and $\mathbf{4}$ of $\tilde{S}_{4}$,

$$
\begin{gathered}
Y_{\hat{\mathbf{2}}}^{\left(\frac{5}{2}\right)}=-\left(Y_{\hat{\mathbf{2}}}^{\left(\frac{1}{2}\right)} Y_{\mathbf{3}}^{(2)}\right)_{\hat{\mathbf{2}}}=\left(\begin{array}{c}
\vartheta_{1}^{5}-5 \vartheta_{1} \vartheta_{2}^{4} \\
\vartheta_{2}^{5}-5 \vartheta_{1}^{4} \vartheta_{2}
\end{array}\right), \\
Y_{\mathbf{4}}^{\left(\frac{5}{2}\right)}=\left(Y_{\hat{\mathbf{2}}}^{\left(\frac{1}{2}\right)} Y_{\mathbf{2}}^{(2)}\right)_{\mathbf{4}}=\left(\begin{array}{c}
\vartheta_{1}\left(\vartheta_{1}^{4}+\vartheta_{2}^{4}\right) \\
2 \sqrt{3} \vartheta_{1}^{3} \vartheta_{2}^{2} \\
-\vartheta_{2}\left(\vartheta_{1}^{4}+\vartheta_{2}^{4}\right) \\
-2 \sqrt{3} \vartheta_{1}^{2} \vartheta_{2}^{3}
\end{array}\right) .
\end{gathered}
$$

The weight 3 modular forms can be arranged into a singlet and two triplets representation of $\tilde{S}_{4}$ as follows,

$$
\begin{aligned}
& Y_{\hat{\mathbf{1}}^{\prime}}^{(3)}=-\frac{1}{6}\left(Y_{\hat{\mathbf{2}}}^{\left(\frac{1}{2}\right)} Y_{\hat{\mathbf{2}}}^{\left(\frac{5}{2}\right)}\right)_{\hat{\mathbf{1}}_{\mathbf{a}}^{\prime}}=\vartheta_{1} \vartheta_{2}\left(\vartheta_{1}^{4}-\vartheta_{2}^{4}\right), \\
& Y_{\hat{\mathbf{3}}}^{(3)}=-\frac{1}{\sqrt{3}}\left(Y_{\hat{\mathbf{2}}}^{\left(\frac{1}{2}\right)} Y_{\mathbf{4}}^{\left(\frac{5}{2}\right)}\right)_{\hat{\mathbf{3}}}=\left(\begin{array}{c}
4 \sqrt{2} \vartheta_{1}^{3} \vartheta_{2}^{3} \\
\vartheta_{1}^{6}+3 \vartheta_{1}^{2} \vartheta_{2}^{4} \\
-\vartheta_{2}^{2}\left(3 \vartheta_{1}^{4}+\vartheta_{2}^{4}\right)
\end{array}\right), \\
& Y_{\hat{\mathbf{3}}^{\prime}}^{(3)}=-\frac{1}{\sqrt{2}}\left(Y_{\hat{\mathbf{2}}}^{\left(\frac{1}{2}\right)} Y_{\hat{\mathbf{2}}}^{\left(\frac{5}{2}\right)}\right)_{\hat{\mathbf{3}}_{\mathrm{s}}^{\prime}}=\left(\begin{array}{c}
2 \sqrt{2} \vartheta_{1} \vartheta_{2}\left(\vartheta_{1}^{4}+\vartheta_{2}^{4}\right) \\
\vartheta_{2}^{6}-5 \vartheta_{1}^{4} \vartheta_{2}^{2} \\
5 \vartheta_{1}^{2} \vartheta_{2}^{4}-\vartheta_{1}^{6}
\end{array}\right) .
\end{aligned}
$$

The linear space of modular forms of weight $7 / 2$ and level 4 has dimension 8 , and it can be decomposed into three $\tilde{S}_{4}$ multiplets $\tilde{\mathbf{2}}^{\prime}, \tilde{\mathbf{2}}$ and $\mathbf{4}^{\prime}$ as follow,

$$
\begin{aligned}
& Y_{\tilde{\mathbf{2}}^{\prime}}^{\left(\frac{7}{2}\right)}=\left(Y_{\hat{\mathbf{2}}}^{\left(\frac{1}{2}\right)} Y_{\hat{\mathbf{1}}^{\prime}}^{(3)}\right)_{\tilde{\mathbf{2}}^{\prime}}=\vartheta_{1} \vartheta_{2}\left(\vartheta_{1}^{4}-\vartheta_{2}^{4}\right)\left(\begin{array}{c}
\vartheta_{1} \\
\vartheta_{2}
\end{array}\right), \\
& Y_{\tilde{\mathbf{2}}}^{\left(\frac{7}{2}\right)}=-\frac{1}{\sqrt{2}}\left(Y_{\hat{\mathbf{2}}}^{\left(\frac{1}{2}\right)} Y_{\hat{\mathbf{3}}}^{(3)}\right)_{\tilde{\mathbf{2}}}=\left(\begin{array}{c}
\vartheta_{2}^{7}+7 \vartheta_{1}^{4} \vartheta_{2}^{3} \\
-\vartheta_{1}^{3}\left(\vartheta_{1}^{4}+7 \vartheta_{2}^{4}\right)
\end{array}\right), \\
& Y_{\mathbf{4}^{\prime}}^{\left(\frac{7}{2}\right)}=\left(Y_{\hat{\mathbf{2}}}^{\left(\frac{1}{2}\right)} Y_{\hat{\mathbf{3}}}^{(3)}\right)_{\mathbf{4}^{\prime}}=\left(\begin{array}{c}
5 \vartheta_{1}^{4} \vartheta_{2}^{3}-\vartheta_{2}^{7} \\
\sqrt{3} \vartheta_{1}^{2} \vartheta_{2}\left(\vartheta_{1}^{4}+3 \vartheta_{2}^{4}\right) \\
5 \vartheta_{1}^{3} \vartheta_{2}^{4}-\vartheta_{1}^{7} \\
\sqrt{3} \vartheta_{1} \vartheta_{2}^{2}\left(3 \vartheta_{1}^{4}+\vartheta_{2}^{4}\right)
\end{array}\right) .
\end{aligned}
$$

At weight 4, we find the following modular multiplets, 


$$
\begin{aligned}
& Y_{\mathbf{1}}^{(4)}=-\left(Y_{\hat{2}}^{\left(\frac{1}{2}\right)} Y_{\tilde{2}}^{\left(\frac{7}{2}\right)}\right)_{\mathbf{1}}=\vartheta_{1}^{8}+14 \vartheta_{1}^{4} \vartheta_{2}^{4}+\vartheta_{2}^{8}, \\
& Y_{\mathbf{2}}^{(4)}=-\left(Y_{\hat{\mathbf{2}}}^{\left(\frac{1}{2}\right)} Y_{\mathbf{4}^{\prime}}^{\left(\frac{7}{2}\right)}\right)_{\mathbf{2}}=\left(\begin{array}{c}
\vartheta_{1}^{8}-10 \vartheta_{1}^{4} \vartheta_{2}^{4}+\vartheta_{2}^{8} \\
4 \sqrt{3} \vartheta_{1}^{2} \vartheta_{2}^{2}\left(\vartheta_{1}^{4}+\vartheta_{2}^{4}\right)
\end{array}\right), \\
& Y_{\mathbf{3}}^{(4)}=\left(Y_{\hat{\mathbf{2}}}^{\left(\frac{1}{2}\right)} Y_{\tilde{\mathbf{2}}}^{\left(\frac{7}{2}\right)}\right)_{\mathbf{3}}=\left(\begin{array}{c}
\vartheta_{2}^{8}-\vartheta_{1}^{8} \\
\sqrt{2} \vartheta_{2}\left(\vartheta_{1}^{7}+7 \vartheta_{1}^{3} \vartheta_{2}^{4}\right) \\
\sqrt{2} \vartheta_{1}\left(\vartheta_{2}^{7}+7 \vartheta_{1}^{4} \vartheta_{2}^{3}\right)
\end{array}\right), \\
& Y_{\mathbf{3}^{\prime}}^{(4)}=\frac{1}{\sqrt{2}}\left(Y_{\hat{\mathbf{2}}}^{\left(\frac{1}{2}\right)} Y_{\tilde{\mathbf{2}}^{\prime}}^{\left(\frac{7}{2}\right)}\right)_{\mathbf{3}^{\prime}}=\vartheta_{1} \vartheta_{2}\left(\vartheta_{1}^{4}-\vartheta_{2}^{4}\right)\left(\begin{array}{c}
\sqrt{2} \vartheta_{1} \vartheta_{2} \\
-\vartheta_{2}^{2} \\
\vartheta_{1}^{2}
\end{array}\right) .
\end{aligned}
$$

We have 10 linearly independent weight 9/2 modular forms which arrange themselves into a $\tilde{S}_{4}$ doublet $\hat{\mathbf{2}}$ and two quartets transforming in the representation $\mathbf{4}$,

$$
\begin{gathered}
Y_{\hat{\mathbf{2}}}^{\left(\frac{9}{2}\right)}=\left(Y_{\hat{\mathbf{2}}}^{\left(\frac{(1)}{2}\right)} Y_{\mathbf{1}}^{(4)}\right)_{\hat{\mathbf{2}}}=\left(\vartheta_{1}^{8}+14 \vartheta_{1}^{4} \vartheta_{2}^{4}+\vartheta_{2}^{8}\right)\left(\begin{array}{c}
\vartheta_{1} \\
\vartheta_{2}
\end{array}\right), \\
Y_{\mathbf{4} I}^{\left(\frac{9}{2}\right)}=\left(Y_{\hat{\mathbf{2}}}^{\left(\frac{1}{2}\right)} Y_{\mathbf{2}}^{(4)}\right)_{\mathbf{4}}=\left(\begin{array}{c}
\vartheta_{1}\left(\vartheta_{1}^{8}-10 \vartheta_{1}^{4} \vartheta_{2}^{4}+\vartheta_{2}^{8}\right) \\
-4 \sqrt{3} \vartheta_{1}^{3} \vartheta_{2}^{2}\left(\vartheta_{1}^{4}+\vartheta_{2}^{4}\right) \\
-\vartheta_{2}\left(\vartheta_{1}^{8}-10 \vartheta_{1}^{4} \vartheta_{2}^{4}+\vartheta_{2}^{8}\right) \\
4 \sqrt{3} \vartheta_{1}^{2} \vartheta_{2}^{3}\left(\vartheta_{1}^{4}+\vartheta_{2}^{4}\right)
\end{array}\right), \\
Y_{\mathbf{4 I I}}^{\left(\frac{9}{2}\right)}=-\frac{1}{\sqrt{2}}\left(Y_{\hat{\mathbf{2}}}^{\left(\frac{1}{2}\right)} Y_{\mathbf{3}}^{(4)}\right)_{\mathbf{4}}=\left(\begin{array}{c}
\vartheta_{1}^{9}-7 \vartheta_{1}^{5} \vartheta_{2}^{4}-2 \vartheta_{1} \vartheta_{2}^{8} \\
-\sqrt{3} \vartheta_{2}^{2}\left(\vartheta_{1}^{7}+7 \vartheta_{1}^{3} \vartheta_{2}^{4}\right) \\
-\vartheta_{2}^{9}+7 \vartheta_{1}^{4} \vartheta_{2}^{5}+2 \vartheta_{1}^{8} \vartheta_{2} \\
\sqrt{3} \vartheta_{1}^{2}\left(\vartheta_{2}^{7}+7 \vartheta_{1}^{4} \vartheta_{2}^{3}\right)
\end{array}\right) .
\end{gathered}
$$

At weight 5, we find the following modular multiplets:

$$
\begin{aligned}
& Y_{\mathbf{2}^{\prime}}^{(5)}=\frac{1}{3}\left(Y_{\hat{\mathbf{2}}}^{\left(\frac{1}{2}\right)} Y_{\mathbf{4} I I}^{\left(\frac{9}{2}\right)}\right)_{\mathbf{2}^{\prime}}=\vartheta_{1} \vartheta_{2}\left(\vartheta_{1}^{4}-\vartheta_{2}^{4}\right)\left(\begin{array}{c}
2 \sqrt{3} \vartheta_{1}^{2} \vartheta_{2}^{2} \\
\vartheta_{1}^{4}+\vartheta_{2}^{4}
\end{array}\right), \\
& Y_{\hat{\mathbf{3}}}^{(5)}=-\frac{1}{\sqrt{3}}\left(Y_{\hat{\mathbf{2}}}^{\left(\frac{1}{2}\right)} Y_{\mathbf{4} I}^{\left(\frac{9}{2}\right)}\right)_{\hat{\mathbf{3}}}=\left(\begin{array}{c}
-8 \sqrt{2} \vartheta_{1}^{3} \vartheta_{2}^{3}\left(\vartheta_{1}^{4}+\vartheta_{2}^{4}\right) \\
\vartheta_{1}^{2}\left(\vartheta_{1}^{8}-14 \vartheta_{1}^{4} \vartheta_{2}^{4}-3 \vartheta_{2}^{8}\right) \\
\vartheta_{2}^{2}\left(3 \vartheta_{1}^{8}+14 \vartheta_{1}^{4} \vartheta_{2}^{4}-\vartheta_{2}^{8}\right)
\end{array}\right), \\
& Y_{\hat{\mathbf{z}}^{\prime} I}^{(5)}=-\left(Y_{\hat{\mathbf{2}}}^{\left(\frac{1}{2}\right)} Y_{\mathbf{4} I}^{\left(\frac{9}{2}\right)}\right)_{\hat{\mathbf{3}}^{\prime}}=\left(\begin{array}{c}
2 \sqrt{2} \vartheta_{1} \vartheta_{2}\left(\vartheta_{1}^{8}-10 \vartheta_{1}^{4} \vartheta_{2}^{4}+\vartheta_{2}^{8}\right) \\
\vartheta_{2}^{2}\left(13 \vartheta_{1}^{8}+2 \vartheta_{1}^{4} \vartheta_{2}^{4}+\vartheta_{2}^{8}\right) \\
-\vartheta_{1}^{2}\left(\vartheta_{1}^{8}+2 \vartheta_{1}^{4} \vartheta_{2}^{4}+13 \vartheta_{2}^{8}\right)
\end{array}\right), \\
& Y_{\hat{\mathbf{3}}^{\prime} I I}^{(5)}=\left(Y_{\hat{\mathbf{2}}}^{\left(\frac{1}{2}\right)} Y_{\mathbf{4} I I}^{\left(\frac{9}{2}\right)}\right)_{\hat{\mathbf{3}}^{\prime}}=\left(\vartheta_{1}^{8}+14 \vartheta_{1}^{4} \vartheta_{2}^{4}+\vartheta_{2}^{8}\right)\left(\begin{array}{c}
\sqrt{2} \vartheta_{1} \vartheta_{2} \\
-\vartheta_{2}^{2} \\
\vartheta_{1}^{2}
\end{array}\right) .
\end{aligned}
$$

There are 12 independent weight $11 / 2$ modular forms of level 4, and they decompose as $\tilde{\mathbf{2}} \oplus \tilde{\mathbf{2}}^{\prime} \oplus \mathbf{4}^{\prime} \oplus \mathbf{4}^{\prime}$ under $\tilde{S}_{4}$,

$$
\begin{aligned}
& Y_{\tilde{\mathbf{2}}}^{\left(\frac{11}{2}\right)}=\frac{1}{\sqrt{2}}\left(Y_{\hat{\mathbf{2}}}^{\left(\frac{1}{2}\right)} Y_{\hat{\mathbf{3}}}^{(5)}\right)_{\tilde{\mathbf{2}}}=\left(\begin{array}{c}
\vartheta_{2}^{3}\left(11 \vartheta_{1}^{8}+22 \vartheta_{1}^{4} \vartheta_{2}^{4}-\vartheta_{2}^{8}\right) \\
\vartheta_{1}^{3}\left(\vartheta_{1}^{8}-22 \vartheta_{1}^{4} \vartheta_{2}^{4}-11 \vartheta_{2}^{8}\right)
\end{array}\right), \\
& Y_{\tilde{\mathbf{2}}^{\prime}}^{\left(\frac{11}{2}\right)}=-\frac{1}{3 \sqrt{2}}\left(Y_{\hat{\mathbf{2}}}^{\left(\frac{1}{2}\right)} Y_{\hat{\mathbf{3}}^{\prime} I}^{(5)}\right)_{\tilde{\mathbf{2}}^{\prime}} \\
& =\vartheta_{1} \vartheta_{2}\left(\vartheta_{1}^{4}-\vartheta_{2}^{4}\right)\left(\begin{array}{c}
\vartheta_{1}^{5}-5 \vartheta_{1} \vartheta_{2}^{4} \\
\vartheta_{2}^{5}-5 \vartheta_{1}^{4} \vartheta_{2}
\end{array}\right) \text {, } \\
& Y_{\mathbf{4}^{\prime} I}^{\left(\frac{11}{2}\right)}=-\frac{1}{\sqrt{3}}\left(Y_{\hat{\mathbf{2}}}^{\left(\frac{1}{2}\right)} Y_{\hat{\mathbf{3}}^{\prime} I}^{(5)}\right)_{\mathbf{4}^{\prime}} \\
& =\left(\begin{array}{c}
\vartheta_{2}^{3}\left(13 \vartheta_{1}^{8}+2 \vartheta_{1}^{4} \vartheta_{2}^{4}+\vartheta_{2}^{8}\right) \\
-\sqrt{3} \vartheta_{1}^{2} \vartheta_{2}\left(\vartheta_{1}^{8}-14 \vartheta_{1}^{4} \vartheta_{2}^{4}-3 \vartheta_{2}^{8}\right) \\
\vartheta_{1}^{3}\left(\vartheta_{1}^{8}+2 \vartheta_{1}^{4} \vartheta_{2}^{4}+13 \vartheta_{2}^{8}\right) \\
\sqrt{3} \vartheta_{1} \vartheta_{2}^{2}\left(3 \vartheta_{1}^{8}+14 \vartheta_{1}^{4} \vartheta_{2}^{4}-\vartheta_{2}^{8}\right)
\end{array}\right), \\
& Y_{\mathbf{4}^{\prime} I I}^{\left(\frac{11}{2}\right)}=\frac{1}{\sqrt{3}}\left(Y_{\hat{\mathbf{2}}}^{\left(\frac{1}{2}\right)} Y_{\hat{\mathbf{3}}^{\prime} I I}^{(5)}\right)_{\mathbf{4}^{\prime}} \\
& =\left(\vartheta_{1}^{8}+14 \vartheta_{1}^{4} \vartheta_{2}^{4}+\vartheta_{2}^{8}\right)\left(\begin{array}{c}
\vartheta_{2}^{3} \\
\sqrt{3} \vartheta_{1}^{2} \vartheta_{2} \\
\vartheta_{1}^{3} \\
\sqrt{3} \vartheta_{1} \vartheta_{2}^{2}
\end{array}\right) .
\end{aligned}
$$

The linear space of weight 6 modular forms has dimension 13 , and it can be decomposed into the direct sum $\mathbf{1} \oplus \mathbf{1}^{\prime} \oplus \mathbf{2} \oplus \mathbf{3} \oplus \mathbf{3} \oplus \mathbf{3}^{\prime}$ :

$$
\begin{aligned}
& Y_{\mathbf{1}}^{(6)}=\left(Y_{\hat{\mathbf{2}}}^{\left(\frac{1}{2}\right)} Y_{\tilde{\mathbf{2}}}^{\left(\frac{11}{2}\right)}\right)_{\mathbf{1}}=\vartheta_{1}^{12}-33 \vartheta_{1}^{8} \vartheta_{2}^{4}-33 \vartheta_{1}^{4} \vartheta_{2}^{8}+\vartheta_{2}^{12}, \\
& Y_{\mathbf{1}^{\prime}}^{(6)}=-\frac{1}{6}\left(Y_{\hat{\mathbf{2}}}^{\left(\frac{1}{2}\right)} Y_{\tilde{\mathbf{2}}^{\prime}}^{\left(\frac{11}{2}\right)}\right)_{\mathbf{1}^{\prime}}=\vartheta_{1}^{2} \vartheta_{2}^{2}\left(\vartheta_{1}^{4}-\vartheta_{2}^{4}\right)^{2},
\end{aligned}
$$$$
Y_{\mathbf{2}}^{(6)}=\left(Y_{\hat{2}}^{\left(\frac{1}{2}\right)} Y_{4^{\prime} I}^{\left(\frac{11}{2}\right)}\right)_{2}=\left(\vartheta_{1}^{8}+14 \vartheta_{1}^{4} \vartheta_{2}^{4}+\vartheta_{2}^{8}\right)\left(\begin{array}{c}
\vartheta_{1}^{4}+\vartheta_{2}^{4} \\
-2 \sqrt{3} \vartheta_{1}^{2} \vartheta_{2}^{2}
\end{array}\right),
$$$$
Y_{\mathbf{3} I}^{(6)}=\frac{1}{\sqrt{2}}\left(Y_{\hat{\mathbf{2}}}^{\left(\frac{1}{2}\right)} Y_{\mathbf{4}^{\prime} I}^{\left(\frac{11}{2}\right)}\right)_{\mathbf{3}}=\left(\begin{array}{c}
\vartheta_{1}^{12}-11 \vartheta_{1}^{8} \vartheta_{2}^{4}+11 \vartheta_{1}^{4} \vartheta_{2}^{8}-\vartheta_{2}^{12} \\
-\sqrt{2} \vartheta_{1}^{3} \vartheta_{2}\left(\vartheta_{1}^{8}-22 \vartheta_{1}^{4} \vartheta_{2}^{4}-11 \vartheta_{2}^{8}\right) \\
\sqrt{2} \vartheta_{1} \vartheta_{2}^{3}\left(11 \vartheta_{1}^{8}+22 \vartheta_{1}^{4} \vartheta_{2}^{4}-\vartheta_{2}^{8}\right)
\end{array}\right) \text {, }
$$$$
Y_{\mathbf{3} I I}^{(6)}=\frac{1}{\sqrt{2}}\left(Y_{\hat{\mathbf{2}}}^{\left(\frac{1}{2}\right)} Y_{\mathbf{4}^{\prime} I I}^{\left(\frac{11}{2}\right)}\right)_{\mathbf{3}}=\left(\vartheta_{1}^{8}+14 \vartheta_{2}^{4} \vartheta_{1}^{4}+\vartheta_{2}^{8}\right)\left(\begin{array}{c}
\vartheta_{1}^{4}-\vartheta_{2}^{4} \\
2 \sqrt{2} \vartheta_{1}^{3} \vartheta_{2} \\
2 \sqrt{2} \vartheta_{1} \vartheta_{2}^{3}
\end{array}\right) \text {, }
$$$$
Y_{\mathbf{3}^{\prime}}^{(6)}=\frac{1}{2 \sqrt{3}}\left(Y_{\hat{\mathbf{2}}}^{\left(\frac{1}{2}\right)} Y_{\mathbf{4}^{\prime} I}^{\left(\frac{11}{2}\right)}\right)_{\mathbf{3}^{\prime}}
$$$$
=\vartheta_{1} \vartheta_{2}\left(\vartheta_{1}^{4}-\vartheta_{2}^{4}\right)\left(\begin{array}{c}
2 \sqrt{2} \vartheta_{1} \vartheta_{2}\left(\vartheta_{1}^{4}+\vartheta_{2}^{4}\right) \\
\vartheta_{2}^{6}-5 \vartheta_{1}^{4} \vartheta_{2}^{2} \\
5 \vartheta_{1}^{2} \vartheta_{2}^{4}-\vartheta_{1}^{6}
\end{array}\right) \text {. }
$$ 
TABLE II. Summary of modular forms of level $N=4$ up to weight 6 , the subscript $\mathbf{r}$ denotes the transformation property under the finite metaplectic group $\tilde{S}_{4}$.

\begin{tabular}{lc}
\hline \hline Modular weight $k / 2$ & Modular forms $Y_{\mathbf{r}}^{\left(\frac{k}{2}\right)}$ \\
\hline$k=1$ & $Y_{\hat{2}}^{\left(\frac{1}{2}\right)}$ \\
$k=2$ & $Y_{\hat{\mathbf{z}}^{\prime}}^{(1)}$ \\
$k=3$ & $Y_{\mathbf{4}^{\prime}}^{\left(\frac{3}{2}\right)}$ \\
$k=4$ & $Y_{\mathbf{2}}^{(2)}, Y_{\mathbf{3}}^{(2)}$ \\
$k=5$ & $Y_{\hat{\mathbf{2}}}^{\left(\frac{5}{2}\right)}, Y_{\mathbf{4}}^{\left(\frac{5}{2}\right)}$ \\
$k=6$ & $Y_{\hat{\mathbf{1}}^{\prime}}^{(3)}, Y_{\hat{\mathbf{3}}}^{(3)}, Y_{\hat{\mathbf{3}}^{\prime}}^{(3)}$ \\
$k=7$ & $Y_{\hat{\mathbf{2}}^{\prime}}^{\left(\frac{7}{2}\right)}, Y_{\hat{\mathbf{2}}}^{\left(\frac{7}{2}\right)}, Y_{\mathbf{4}^{\prime}}^{\left(\frac{7}{2}\right)}$ \\
$k=8$ & $Y_{\mathbf{1}}^{(4)}, Y_{\mathbf{2}}^{(4)}, Y_{\mathbf{3}}^{(4)}, Y_{\mathbf{3}^{\prime}}^{(4)}$ \\
$k=9$ & $Y_{\hat{\mathbf{2}}}^{\left(\frac{9}{2}\right)}, Y_{\mathbf{4} I}^{\left(\frac{9}{2}\right)}, Y_{\mathbf{4}_{I I I}^{\left(\frac{9}{2}\right)}}^{(5)}$ \\
$k=10$ & $Y_{\mathbf{2}^{\prime}}^{(5)}, Y_{\hat{\mathbf{3}}}^{(5)}, Y_{\hat{\mathbf{z}}^{\prime} I}^{(5)}, Y_{\hat{\mathbf{z}}^{\prime} I I}^{(5)}$ \\
$k=11$ & $Y_{\tilde{\mathbf{2}}}^{\left(\frac{11}{2}\right)}, Y_{\hat{\mathbf{2}}^{\prime}}^{\left(\frac{11}{2}\right)}, Y_{\mathbf{4}^{\prime} I}^{\left(\frac{11}{2}\right)}, Y_{\mathbf{4}^{\prime} I I}^{\left(\frac{11}{2}\right)}$ \\
$k=12$ & $Y_{\mathbf{1}}^{(6)}, Y_{\mathbf{1}^{\prime}}^{(6)}, Y_{\mathbf{2}}^{(6)}, Y_{\mathbf{3} I}^{(6)}, Y_{\mathbf{3}}^{(6)}, Y_{\mathbf{3}^{\prime}}^{(6)}$ \\
\hline \hline
\end{tabular}

We summarize the modular forms of level 4 up to weight 6 in Table II. If the complex modulus $\tau$ is stabilized at certain points, it would be invariant under some modular transformation and some residual modular flavor symmetry would be preserved [16,25]. It is wellknown that there are only four fixed points $\tau_{S}=i, \tau_{S T}=$ $\omega, \tau_{T S}=-\omega^{2}, \tau_{T}=+i \infty$ with $\omega=e^{2 i \pi / 3}$ in the fundamental domain of $\mathrm{SL}_{2}(\mathbb{Z})$ group [16,25]. In the following, we give the vacuum alignment of half weight modular form $Y_{\hat{2}}^{\left(\frac{1}{2}\right)}(\tau)$ at these fixed points which could be useful to modular flavor model building [25],

$$
\begin{aligned}
& Y_{\hat{\mathbf{2}}}^{\left(\frac{1}{2}\right)}\left(\tau_{S}\right)=Y_{S}\left(\begin{array}{c}
1 \\
1-\sqrt{2}
\end{array}\right), \\
& Y_{\hat{\mathbf{2}}}^{\left(\frac{1}{2}\right)}\left(\tau_{S T}\right)=Y_{S T}\left(\begin{array}{c}
\omega \\
-1-e^{\frac{5 \pi i}{6}}
\end{array}\right), \\
& Y_{\hat{\mathbf{2}}}^{\left(\frac{1}{2}\right)}\left(\tau_{T}\right)=Y_{T}\left(\begin{array}{c}
1 \\
0
\end{array}\right), \\
& Y_{\hat{\mathbf{2}}}^{\left(\frac{1}{2}\right)}\left(\tau_{T S}\right)=Y_{T S}\left(\begin{array}{c}
\omega^{2} \\
-1+e^{\frac{\pi i}{6}}
\end{array}\right),
\end{aligned}
$$

with $Y_{S}=1.00373, Y_{S T}=-0.49567-0.85852 i, Y_{T S}=$ $-0.49567+0.85852 i$ and $Y_{T}=1$. The alignments of higher weight modular forms at fixed points can be easily obtained from Eq. (54) and the concrete expressions of higher weight modular forms given above.

\section{MODEL FOR LEPTON MASSES AND FLAVOR MIXING}

As shown in Sec. II, in order to consistently define halfintegral weight modular forms, a multiplier is necessary and the modular group $\mathrm{SL}_{2}(\mathbb{Z})$ should be extended to the metaplectic group $\mathrm{Mp}_{2}(\mathbb{Z})$ which is the double covering of $\mathrm{SL}_{2}(\mathbb{Z})$. As a result, we need to generalize the original modular invariant supersymmetric theory [1] to metaplectic modular invariant theory.

\section{A. Metaplectic modular invariant theory}

We adopt the framework of the $\mathcal{N}=1$ global supersymmetry, the most general form of the action is

$$
\begin{aligned}
\mathcal{S}= & \int d^{4} x d^{2} \theta d^{2} \bar{\theta} K\left(\Phi_{I}, \bar{\Phi}_{I}, \tau, \bar{\tau}\right) \\
& +\int d^{4} x d^{2} \theta \mathcal{W}\left(\Phi_{I}, \tau\right)+\text { H.c. },
\end{aligned}
$$

where $K\left(\Phi_{I}, \bar{\Phi}_{I}, \tau, \bar{\tau}\right)$ is the Kähler potential, $\mathcal{W}\left(\Phi_{I}, \tau\right)$ is the superpotential, and $\Phi_{I}$ denotes a set of chiral supermultiplets. The metaplectic group acts on the modulus $\tau$ and the superfield $\Phi_{I}$ in a certain way $[1,69,70]$. Analogous to [1], we assume the supermultiplet $\Phi_{I}$ transforms in a representation $\rho_{I}$ of the finite metaplectic group $\tilde{\Gamma}_{4 N}$ with a weight $-k_{I} / 2$,

$$
\begin{aligned}
\tau & \rightarrow \tilde{\gamma} \tau=\frac{a \tau+b}{c \tau+d}, \\
\Phi_{I} & \rightarrow \phi^{-k_{I}}(\gamma, \tau) \rho_{I}(\tilde{\gamma}) \Phi_{I} \quad \text { with } \quad \tilde{\gamma}=(\gamma, \phi(\gamma, \tau)) \in \tilde{\Gamma},
\end{aligned}
$$

where $\gamma=\left(\begin{array}{l}a b \\ c d\end{array}\right)$ and $\phi(\gamma, \tau)=\epsilon(c \tau+d)^{1 / 2}, \rho_{I}(\tilde{\gamma})$ is the unitarity representation matrix of $\tilde{\gamma}$, and $k_{I}$ is a generic nonnegative integer. The supermultiplets $\Phi_{I}$ are not modular forms, therefore, there is no restriction on the possible value of $k_{I}$. We can see that the combination of any two metaplectic transformations $\tilde{\gamma}_{1}$ and $\tilde{\gamma}_{2}$ is also a metaplectic transformation,

$$
\begin{aligned}
\tau & \rightarrow \tilde{\gamma}_{1}\left(\tilde{\gamma}_{2} \tau\right)=\left(\tilde{\gamma_{1}} \tilde{\gamma_{2}}\right) \tau, \\
\Phi_{I} & \rightarrow \phi^{-k_{I}}\left(\gamma_{1}, \gamma_{2} \tau\right) \phi^{-k_{I}}\left(\gamma_{2}, \tau\right) \rho_{I}\left(\tilde{\gamma}_{1}\right) \rho_{I}\left(\tilde{\gamma}_{2}\right) \Phi_{I} \\
& =\phi^{-k_{I}}\left(\gamma_{1}, \gamma_{2} \tau\right) \phi^{-k_{I}}\left(\gamma_{2}, \tau\right) \rho_{I}\left(\tilde{\gamma}_{1} \tilde{\gamma}_{2}\right) \Phi_{I} .
\end{aligned}
$$

If we are still confined to the original modular group $\mathrm{SL}_{2}(\mathbb{Z})$ instead of $\mathrm{Mp}_{2}(\mathbb{Z})$. Then the modular transformation of matter fields is $\Phi_{I} \rightarrow J_{k_{I} / 2}^{-1}(\gamma, \tau) \rho(\gamma) \Phi_{I}$, the combination of two half-integral weight modular transformations would be unequal to a third half-integral weight modular transformation due to presence of the factor $\zeta_{k_{I} / 2}\left(\gamma_{1}, \gamma_{2}\right)$ which arises because of the identity in Eq. (8a), so that the modular transformation is not well defined. For this reason, 
it is insufficient to simply change the modular weight to a rational or real number when discussing modular transformations of rational or real weight, the classical modular group $\mathrm{SL}_{2}(\mathbb{Z})$ should be enhanced to its metaplectic covering group. The action $\mathcal{S}$ is required invariant under the metaplectic transformation given in Eq. (56). The Kähler potential $K\left(\Phi_{I}, \bar{\Phi}_{I}, \tau, \bar{\tau}\right)$ is a real gauge-invariant function of the chiral supermultiplets $\Phi_{I}$ and their conjugates. A minimal choice of Kähler potential is

$$
\begin{aligned}
K\left(\Phi_{I}, \bar{\Phi}_{I}, \tau, \bar{\tau}\right)= & -h \Lambda^{2} \log (-i \tau+i \bar{\tau}) \\
& +\sum_{I}(-i \tau+i \bar{\tau})^{-\frac{k_{I}}{2}}\left|\Phi_{I}\right|^{2},
\end{aligned}
$$

where $h$ is a positive constant. $K\left(\Phi_{I}, \bar{\Phi}_{I}, \tau, \bar{\tau}\right)$ is invariant up to a Kähler transformation under the metaplectic transformation. The superpotential $\mathcal{W}\left(\Phi_{I}, \tau\right)$ can be expanded in a power series of the supermultiplets $\Phi_{I}$ :

$$
\mathcal{W}\left(\Phi_{I}, \tau\right)=\sum_{n} Y_{I_{1} \ldots I_{n}}(\tau) \Phi_{I_{1}} \ldots \Phi_{I_{n}}
$$

Invariance of $\mathcal{W}\left(\Phi_{I}, \tau\right)$ under the metaplectic transformation in Eq. (56) entails that the function $Y_{I_{1} \ldots I_{n}}(\tau)$ should be a modular form of weight $k_{Y} / 2$ and level $4 N$ transforming in the representation $\rho_{Y}$ of $\tilde{\Gamma}_{4 N}$,

$$
Y_{I_{1} \ldots I_{n}}(\gamma \tau)=\phi^{k_{Y}}(\gamma, \tau) \rho_{Y}(\tilde{\gamma}) Y_{I_{1} \ldots I_{n}}(\tau), \quad \tilde{\gamma}=(\gamma, \phi(\gamma, \tau))
$$

The modular weight $k_{Y} / 2$ and the representation $\rho_{Y}$ should satisfy the conditions

$k_{Y}=k_{I_{1}}+\cdots+k_{I_{n}}, \quad \rho_{Y} \otimes \rho_{I_{1}} \otimes \cdots \otimes \rho_{I_{n}} \supset \mathbf{1}$,

where 1 refers to invariant singlet of $\tilde{\Gamma}_{4 N}$.

\section{B. Models based on $\tilde{\boldsymbol{S}}_{\mathbf{4}}$}

In this section, we shall construct lepton models based on the finite metaplectic modular group $\tilde{\Gamma}_{4} \cong \tilde{S}_{4}$. In the representations $\mathbf{1}, \mathbf{1}^{\prime}, \hat{\mathbf{1}}, \hat{\mathbf{1}}^{\prime}, \mathbf{2}, \mathbf{2}^{\prime}, \mathbf{3}, \mathbf{3}^{\prime}, \hat{\mathbf{3}}$ and $\hat{\mathbf{3}}^{\prime}$, the generator $\tilde{R}= \pm 1$ and therefore $\tilde{S}_{4}$ and the homogeneous finite modular group $S_{4}^{\prime}$ are represented by the same set of matrices. As a consequence, all the $S_{4}^{\prime}$ modular models obviously can be reproduced from the metaplectic modular group $\tilde{S}_{4}$, in particular the successful $S_{4}^{\prime}$ models given in our previous work [53] can be obtained here. In the following, we shall explore new models beyond $S_{4}^{\prime}$, and half-integral weight modular forms would be involved. The neutrino masses originate from the effective Weinberg operator or the type-I seesaw mechanism, and both
TABLE III. Transformation properties of the leptonic matter fields under the finite metaplectic group $\tilde{\Gamma}_{4}$, and the modular weight $k_{I} / 2$ assignments for each model. The Higgs fields $H_{u}$ and $H_{d}$ are assigned to be invariant singlet $\mathbf{1}$ of $\tilde{S}_{4}$ with vanishing modular weight.

\begin{tabular}{cccccccccc}
\hline \hline & $E_{1}^{c}$ & $E_{2}^{c}$ & $E_{3}^{c}$ & $L$ & $N_{1}^{c}$ & $N_{2}^{c}$ & $N_{3}^{c}$ & $H_{u}$ & $H_{d}$ \\
\hline Model I & $\tilde{S}_{4}$ & $\hat{\mathbf{2}}$ & $\mathbf{1}$ & $\hat{\mathbf{3}}$ & $\ldots$ & $\ldots$ & $\ldots$ & $\mathbf{1}$ & $\mathbf{1}$ \\
& $k_{I} / 2$ & $3 / 2$ & 0 & 1 & $\ldots$ & $\ldots$ & $\ldots$ & 0 & 0 \\
Model II & $\tilde{S}_{4}$ & $\tilde{\mathbf{2}}$ & $\hat{\mathbf{1}}^{\prime}$ & $\hat{\mathbf{3}}$ & & $\mathbf{3}^{\prime}$ & & $\mathbf{1}$ & $\mathbf{1}$ \\
& $k_{I} / 2$ & $3 / 2$ & 0 & 1 & & 1 & & 0 & 0 \\
Model III & $\tilde{S}_{4}$ & $\mathbf{1}$ & $\mathbf{1}$ & $\hat{\mathbf{1}}$ & $\mathbf{3}$ & $\tilde{\mathbf{2}}$ & $\ldots$ & $\mathbf{1}$ & $\mathbf{1}$ \\
& $k_{I} / 2$ & 1 & 3 & 4 & 1 & $3 / 2$ & $\ldots$ & 0 & 0 \\
\hline \hline
\end{tabular}

scenarios of three right-handed neutrinos and two righthanded neutrinos are considered in the type-I seesaw mechanism. No flavon field other than the modulus $\tau$ is introduced in these models such that $\tau$ is the unique source of flavor symmetry breaking. The three models would be presented in the following one by one, the field content and their transformation properties under $\tilde{S}_{4}$ and weights are summarized in Table III.

\section{Model I: Neutrino masses from Weinberg operator}

In this model, the neutrino masses are described by the Weinberg operator. The left-handed leptons $L$ are assigned to a triplet $\hat{\mathbf{3}}$, the first two generations of right-handed charged leptons $E_{1}^{c}$ and $E_{2}^{c}$ transform as a doublet $\hat{\mathbf{2}}$ of $\tilde{S}_{4}$. For convenience, we use the subscript " $D$ " to denote the doublet assignment, i.e., $E_{D}^{c} \equiv\left(E_{1}^{c}, E_{2}^{c}\right)^{T}$. The third righthanded charged lepton $E_{3}^{c}$ is invariant under $\tilde{S}_{4}$. Notice that the three right-handed charged leptons are generally assumed to transform as singlets of the modular symmetry to fit the hierarchical charged lepton masses, and they are assigned to a triplet under modular symmetry in few models. The modular weights of the lepton superfields are set to

$$
k_{L} / 2=1, \quad k_{E_{D}^{c}} / 2=\frac{3}{2}, \quad k_{E_{3}^{c}} / 2=0 .
$$

Thus the modular invariant superpotentials for lepton masses read as follows,

$$
\begin{aligned}
\mathcal{W}_{e}= & \alpha\left(E_{D}^{c} L Y_{\hat{\mathbf{2}}}^{\left(\frac{5}{2}\right)}\right)_{\mathbf{1}} H_{d}+\beta\left(E_{D}^{c} L Y_{\mathbf{4}}^{\left(\frac{5}{2}\right)}\right)_{\mathbf{1}} H_{d} \\
& +\gamma\left(E_{3}^{c} L Y_{\hat{\mathbf{3}}^{\prime}}^{(1)}\right)_{\mathbf{1}} H_{d}, \\
\mathcal{W}_{\nu}= & \frac{g_{1}}{\Lambda}\left(L L Y_{\mathbf{2}}^{(2)}\right)_{\mathbf{1}} H_{u} H_{u}+\frac{g_{2}}{\Lambda}\left(L L Y_{\mathbf{3}}^{(2)}\right)_{\mathbf{1}} H_{u} H_{u} .
\end{aligned}
$$

With the CG coefficients of $\tilde{S}_{4}$ group in Appendix B, the charged lepton and neutrino mass matrices read as 


$$
\begin{aligned}
& M_{e}=\left(\begin{array}{ccc}
\alpha Y_{\hat{\mathbf{2}}, 2}^{\left(\frac{5}{2}\right)}-\sqrt{2} \beta Y_{\mathbf{4}, 3}^{\left(\frac{5}{2}\right)} & \sqrt{2} \alpha Y_{\hat{\mathbf{2}}, 1}^{\left(\frac{5}{2}\right)}-\beta Y_{\mathbf{4}, 1}^{\left(\frac{5}{2}\right)} & -\sqrt{3} \beta Y_{\mathbf{4}, 2}^{\left(\frac{5}{2}\right)} \\
\alpha Y_{\hat{\mathbf{2}}, 1}^{\left(\frac{5}{2}\right)}+\sqrt{2} \beta Y_{\mathbf{4}, 1}^{\left(\frac{5}{2}\right)} & -\sqrt{3} \beta Y_{\mathbf{4}, 4}^{\left(\frac{5}{2}\right)} & -\sqrt{2} \alpha Y_{\hat{\mathbf{2}}, 2}^{\left(\frac{5}{2}\right)}-\beta Y_{\mathbf{4}, 3}^{\left(\frac{5}{2}\right)} \\
\gamma Y_{\hat{\mathbf{3}}^{\prime}, 1}^{(1)} & \gamma Y_{\hat{\mathbf{3}}^{\prime}, 3}^{(1)} & \gamma Y_{\hat{\mathbf{3}}^{\prime}, 2}^{(1)}
\end{array}\right) v_{d}, \\
& M_{\nu}=\left(\begin{array}{ccc}
-2 g_{1} Y_{\mathbf{2}, 2}^{(2)} & g_{2} Y_{\mathbf{3}, 2}^{(2)} & -g_{2} Y_{\mathbf{3}, 3}^{(2)} \\
g_{2} Y_{\mathbf{3}, 2}^{(2)} & \sqrt{3} g_{1} Y_{\mathbf{2}, 1}^{(2)}+g_{2} Y_{\mathbf{3}, 1}^{(2)} & g_{1} Y_{\mathbf{2}, 2}^{(2)} \\
-g_{2} Y_{\mathbf{3}, 3}^{(2)} & g_{1} Y_{\mathbf{2}, 2}^{(2)} & \sqrt{3} g_{1} Y_{\mathbf{2}, 1}^{(2)}-g_{2} Y_{\mathbf{3}, 1}^{(2)}
\end{array}\right) \frac{v_{u}^{2}}{\Lambda} .
\end{aligned}
$$

where $Y_{\mathbf{r}, n}^{(w)}$ denotes the $n$th component of weight $w$ modular multiplets $Y_{\mathbf{r}}^{(w)}$. The phases of $\alpha, \gamma$ and $g_{1}$ can be removed by field redefinition while $\beta$ and $g_{2}$ are generally complex numbers. Thus this model makes use of three real positive parameters $\alpha, \gamma, g_{1}$ and two complex parameter $\beta, g_{2}$ as well as the complex modulus $\tau$ to describe all the lepton masses and Pontecorvo-Maki-Nakagawa-Sakata (PMNS) matrix including three charged lepton masses $m_{e, \mu, \tau}$, three light neutrino masses $m_{1,2,3}$, three lepton mixing angles $\theta_{12}, \theta_{13}, \theta_{23}$, the Dirac $C P$ violation phase $\delta_{C P}$ and the Majorana $C P$ phases $\alpha_{21}$ and $\alpha_{31}$. Furthermore, the effective mass $m_{\beta}$ in beta decay, $m_{\beta \beta}$ in neutrinoless double beta decay and the neutrino mass sum measured by Planck can be determined, as shown below. Since normal ordering neutrino mass spectrum is slightly favored over the inverted ordering, we shall be concerned with the normal ordering case in the following. A good agreement between data and predictions is obtained for the following values of the free parameters:

$$
\begin{aligned}
\langle\tau\rangle & =0.09865+1.00342 i, \quad|\beta / \alpha|=1.41137, \quad \arg (\beta / \alpha)=1.99681 \pi, \\
\gamma / \alpha & =72.34910, \quad\left|g_{2} / g_{1}\right|=0.64819, \quad \arg \left(g_{2} / g_{1}\right)=0.57182 \pi, \\
\alpha v_{d} & =15.71110 \mathrm{MeV}, \quad \frac{g_{1} v_{u}^{2}}{\Lambda}=22.95000 \mathrm{meV} .
\end{aligned}
$$

The lepton masses and mixing parameters are determined to be

$$
\begin{aligned}
\sin ^{2} \theta_{12} & =0.31004, \quad \sin ^{2} \theta_{13}=0.022368, \quad \sin ^{2} \theta_{23}=0.56282, \quad \delta_{C P}=1.6055 \pi, \\
\alpha_{21} & =0.1831 \pi, \quad \alpha_{31}=0.2359 \pi, \quad m_{e} / m_{\mu}=0.00480, \quad m_{\mu} / m_{\tau}=0.05649 \\
m_{1} & =31.5143 \mathrm{meV}, \quad m_{2}=32.6658 \mathrm{meV}, \quad m_{3}=59.3347 \mathrm{meV} \\
m_{\beta} & =32.7427 \mathrm{meV}, \quad m_{\beta \beta}=28.7321 \mathrm{meV} .
\end{aligned}
$$

It is remarkable that all observables are within the $1 \sigma$ experimental range [71], and we adopt the ratios of the charged lepton masses from [1]

$$
m_{e} / m_{\mu}=0.0048 \pm 0.0002, \quad m_{\mu} / m_{\tau}=0.0565 \pm 0.0045
$$

Realistic values of charged lepton masses can be achieved by properly choosing the value of the overall scale $\alpha v_{d}$. Note that the mass hierarchy between electron and muon arises from the cooperation of the $\alpha$ and $\beta$ terms in Eq. (63). Here we adopt the particle data group convention for the mixing angles and $C P$ violation phases [72]. The lepton mixing matrix in the standard parametrization is written as

$$
U=\left(\begin{array}{ccc}
c_{12} c_{13} & s_{12} c_{13} & s_{13} e^{-i \delta_{C P}} \\
-s_{12} c_{23}-c_{12} s_{13} s_{23} e^{i \delta_{C P}} & c_{12} c_{23}-s_{12} s_{13} s_{23} e^{i \delta_{C P}} & c_{13} s_{23} \\
s_{12} s_{23}-c_{12} s_{13} c_{23} e^{i \delta_{C P}} & -c_{12} s_{23}-s_{12} s_{13} c_{23} e^{i \delta_{C P}} & c_{13} c_{23}
\end{array}\right) Q,
$$

where $c_{i j} \equiv \cos \theta_{i j}, s_{i j} \equiv \sin \theta_{i j}, \delta_{C P}$ is the Dirac $C P$ phase, and $Q$ is a diagonal Majorana phase matrix. If all of the three neutrinos have nonzero masses, then the phase matrix is given by $Q=\operatorname{diag}\left(1, e^{i \frac{\alpha_{21}}{2}}, e^{i^{i \frac{\alpha_{31}}{2}}}\right)$. If the lightest neutrino is predicted to be massless, i.e., $m_{1}=0$ (see Model III), one of the Majorana neutrino phase is unphysical, and we can parametrize the phase matrix as $Q=\operatorname{diag}\left(1, e^{i \phi}, 1\right)$, where $\alpha_{21}, \alpha_{31}$ or $\phi$ are called Majorana $C P$ phases. From the predicted values of 
mixing angles and neutrino masses, one can determine the effective neutrino masses $m_{\beta}$ probed by direct kinematic search in beta decay and the effective mass $m_{\beta \beta}$ in neutrinoless double beta decay. Note that $m_{\beta}$ is independent of the $C P$ violation phase and it is defined by

$$
m_{\beta}=\sqrt{m_{1}^{2} \cos ^{2} \theta_{12} \cos ^{2} \theta_{13}+m_{2}^{2} \sin ^{2} \theta_{12} \cos ^{2} \theta_{13}+m_{3}^{2} \sin ^{2} \theta_{13}} .
$$

The latest bound is $m_{\beta}<1.1 \mathrm{eV}$ at $90 \%$ confidence level from KATRIN [73]. Our prediction $m_{\beta}=32.7427 \mathrm{meV}$ is far below the future sensitivity of KATRIN. The decay rate of the neutrinoless double beta decay is proportional to the square of the effective Majorana mass $m_{\beta \beta}$ defined as

$$
\begin{aligned}
m_{\beta \beta}= & \mid m_{1} \cos ^{2} \theta_{12} \cos ^{2} \theta_{13}+m_{2} \sin ^{2} \theta_{12} \cos ^{2} \theta_{13} e^{i \alpha_{21}} \\
& +m_{3} \sin ^{2} \theta_{13} e^{i\left(\alpha_{31}-2 \delta_{C P}\right)} \mid,
\end{aligned}
$$

in which all mixing parameters except $\theta_{23}$ are involved. For $m_{1}=0$, it can be simply written as

$m_{\beta \beta}=\left|m_{2} \sin ^{2} \theta_{12} \cos ^{2} \theta_{13} e^{i \phi}+m_{3} \sin ^{2} \theta_{13} e^{-i 2 \delta_{C P}}\right|$.

The current experimental bound from KamLAND-Zen is $m_{\beta \beta}<(61-165) \mathrm{meV}$ [74]. The predicted value $m_{\beta \beta}=$ $28.7321 \mathrm{meV}$ is within the reach of the next generation high sensitive neutrinoless double beta decay experiments. The most stringent bound on the neutrino mass sum is $\sum_{i} m_{i}<(120 \sim 600) \mathrm{meV}$ at $95 \%$ confidence level from Plank [75]. In this model, the neutrino mass sum is predicted to be $\sum_{i} m_{i}=123.515 \mathrm{meV}$ which is slightly larger than the upper limit of Planck. We perform a comprehensive numerical analysis for this model. The parameters $|\beta / \alpha|, \gamma / \alpha,\left|g_{2} / g_{1}\right|$ are treated as random numbers between 0 and $10^{4}$, the phases $\arg (\beta / \alpha)$ and $\arg \left(g_{2} / g_{1}\right)$ freely vary in the range $[0,2 \pi]$, and the complex modulus $\tau$ is restricted in the fundamental domain $\mathcal{D}=\left\{\tau\left|\operatorname{Im}(\tau)>0, \operatorname{Re}(\tau) \leq \frac{1}{2},\right| \tau \mid \geq 1\right\}$. The overall mass scales $\alpha v_{d}$ and $g_{1} v_{u}^{2} / \Lambda$ are fixed by the electron masses and the neutrino mass squared difference $\Delta m_{21}^{2}$. In order to estimate the goodness-of-fit of a set of chosen values of the input parameters, we use a $\chi^{2}$ function defined in the usual way for the dimensionless observables $m_{e} / m_{\mu}, m_{\mu} / m_{\tau}$, $\Delta m_{21}^{2} / \Delta m_{31}^{2}, \sin ^{2} \theta_{12}, \sin ^{2} \theta_{13}$, and $\sin ^{2} \theta_{23}$. We require the predictions for all these observables lie in the experimentally preferred $3 \sigma$ regions, and the neutrino masses upper bounds $\sum_{i} m_{i}<600 \mathrm{meV}$ from Plank [75] and $m_{\beta \beta}<165 \mathrm{meV}$ from KamLAND-Zen [74] are imposed. The correlations among the input parameters and the observable quantities are displayed in Fig. 1, and the allowed regions of the free parameters and observables are listed in Table IV. It is worth noting that $\arg (\beta / \alpha)$ is very close to 0 or $\pi$.

\section{Model II: Neutrino masses from seesaw mechanism with three right-handed neutrinos}

The neutrino masses arise from type-I seesaw mechanism in this model, and three right-handed neutrinos are introduced to transform as a triplet $\mathbf{3}^{\prime}$ of $\tilde{S}_{4}$. The left-handed leptons $L$ are assigned to a triplet $\mathbf{3}$, the right-handed charged leptons $E_{D}^{c}$ and $E_{3}^{c}$ are assigned to transform as $\tilde{\mathbf{2}}$ and $\hat{\mathbf{1}}^{\prime}$ respectively under $\tilde{S}_{4}$. We take the modular weights of the lepton superfields to be

$$
k_{L} / 2=1, \quad k_{E_{D}^{c}} / 2=\frac{3}{2}, k_{E_{3}^{c}} / 2=4, k_{N^{c}} / 2=1 .
$$

The superpotentials for lepton masses are given by,

$$
\begin{aligned}
& \mathcal{W}_{e}=\alpha\left(E_{D}^{c} L Y_{\hat{\mathbf{2}}}^{\left(\frac{5}{2}\right)}\right)_{\mathbf{1}} H_{d}+\beta\left(E_{D}^{c} L Y_{\mathbf{4}}^{\left(\frac{5}{2}\right)}\right)_{\mathbf{1}} H_{d}+\gamma\left(E_{3}^{c} L Y_{\hat{\mathbf{3}}}^{(5)}\right)_{\mathbf{1}} H_{d} \\
& \mathcal{W}_{\nu}=g_{1}\left(N^{c} L Y_{\mathbf{2}}^{(2)}\right)_{\mathbf{1}} H_{u}+g_{2}\left(N^{c} L Y_{\mathbf{3}}^{(2)}\right)_{\mathbf{1}} H_{u}+\Lambda\left(N^{c} N^{c} Y_{\mathbf{2}}^{(2)}\right)_{\mathbf{1}},
\end{aligned}
$$

which lead to the following charged lepton and neutrino mass matrices

$$
M_{e}=\left(\begin{array}{ccc}
\alpha Y_{\hat{\mathbf{2}}, 2}^{\left(\frac{5}{2}\right)}-\sqrt{2} \beta Y_{\mathbf{4}, 3}^{\left(\frac{5}{2}\right)} & \sqrt{2} \alpha Y_{\hat{\mathbf{2}}, 1}^{\left(\frac{5}{2}\right)}-\beta Y_{\mathbf{4}, 1}^{\left(\frac{5}{2}\right)} & -\sqrt{3} \beta Y_{\mathbf{4}, 2}^{\left(\frac{5}{2}\right)} \\
\alpha Y_{\hat{\mathbf{2}}, 1}^{\left(\frac{5}{2}\right)}+\sqrt{2} \beta Y_{\mathbf{4}, 1}^{\left(\frac{5}{2}\right)} & -\sqrt{3} \beta Y_{\mathbf{4}, 4}^{\left(\frac{5}{2}\right)} & -\sqrt{2} \alpha Y_{\hat{\mathbf{2}}, 2}^{\left(\frac{5}{2}\right)}-\beta Y_{\mathbf{4}, 3}^{\left(\frac{5}{2}\right)} \\
\gamma Y_{\hat{\mathbf{3}}, 1}^{(5)} & \gamma Y_{\hat{\mathbf{3}}, 3}^{(5)} & \gamma Y_{\hat{\mathbf{3}}, 2}^{(5)}
\end{array}\right) v_{d},
$$



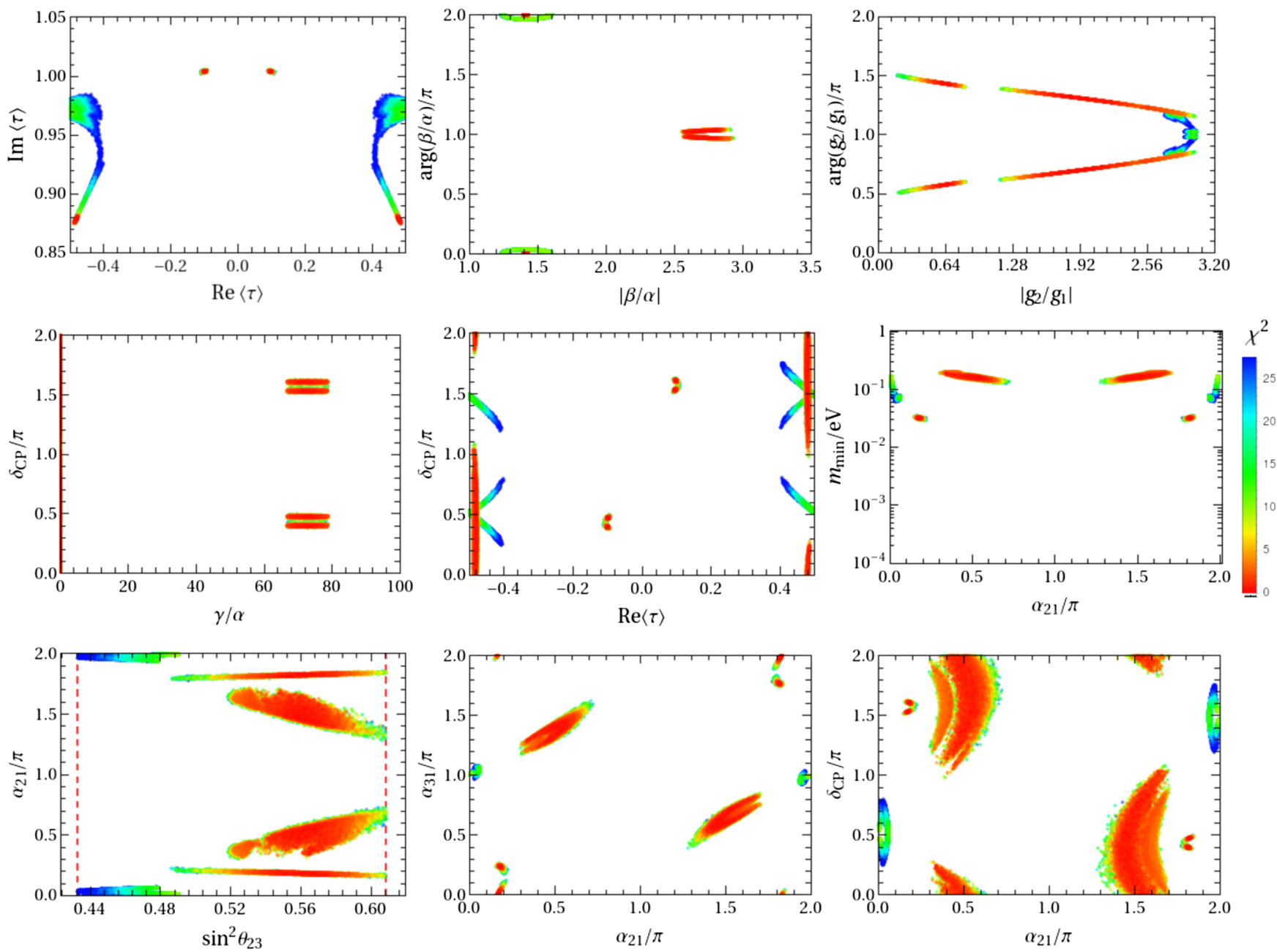

FIG. 1. The predicted correlations among the input free parameters, neutrino mixing angles and $C P$ violating phases in the Model I. The plots displays only the points which reproduce $\Delta m_{21}^{2}, \Delta m_{31}^{2}$ and all the three mixing angles within the $3 \sigma$ regions [71]. The bounds on charged lepton mass in Eq. (67) and the bounds on neutrino masses $\sum_{i} m_{i}<600 \mathrm{meV}, m_{\beta \beta}<165 \mathrm{meV}$ are required to be fulfilled.

$$
\begin{aligned}
M_{N} & =\left(\begin{array}{ccc}
2 Y_{\mathbf{2}, 1}^{(2)} & 0 & 0 \\
0 & \sqrt{3} Y_{\mathbf{2}, 2}^{(2)} & -Y_{\mathbf{2}, 1}^{(2)} \\
0 & -Y_{\mathbf{2}, 1}^{(2)} & \sqrt{3} Y_{\mathbf{2}, 2}^{(2)}
\end{array}\right) \Lambda, \\
M_{D} & =\left(\begin{array}{ccc}
-2 g_{1} Y_{\mathbf{2}, 2}^{(2)} & g_{2} Y_{\mathbf{3}, 2}^{(2)} & -g_{2} Y_{\mathbf{3}, 3}^{(2)} \\
g_{2} Y_{\mathbf{3}, 2}^{(2)} & \sqrt{3} g_{1} Y_{\mathbf{2}, 1}^{(2)}+g_{2} Y_{\mathbf{3}, 1}^{(2)} & g_{1} Y_{\mathbf{2}, 2}^{(2)} \\
-g_{2} Y_{\mathbf{3}, 3}^{(2)} & g_{1} Y_{\mathbf{2}, 2}^{(2)} & \sqrt{3} g_{1} Y_{\mathbf{2}, 1}^{(2)}-g_{2} Y_{\mathbf{3}, 1}^{(2)}
\end{array}\right) v_{u} \cdot
\end{aligned}
$$

The parameters $\alpha, \gamma$ and $g_{1}$ can be taken real since their phases are unphysical, nevertheless the phases of $\beta$ and $g_{2}$ can not be absorbed into lepton fields. The overall scale of the light neutrino mass matrix is the combination $g_{1}^{2} v_{u}^{2} / \Lambda$, consequently, this model effectively depends on 9 free real parameters including the real and imaginary parts of $\tau$ at low energy. We get a good agreement between the model and the data by the parameter choice: 
TABLE IV. The predictions for the allowed ranges of the input parameters and observables in the Model I and Model II.

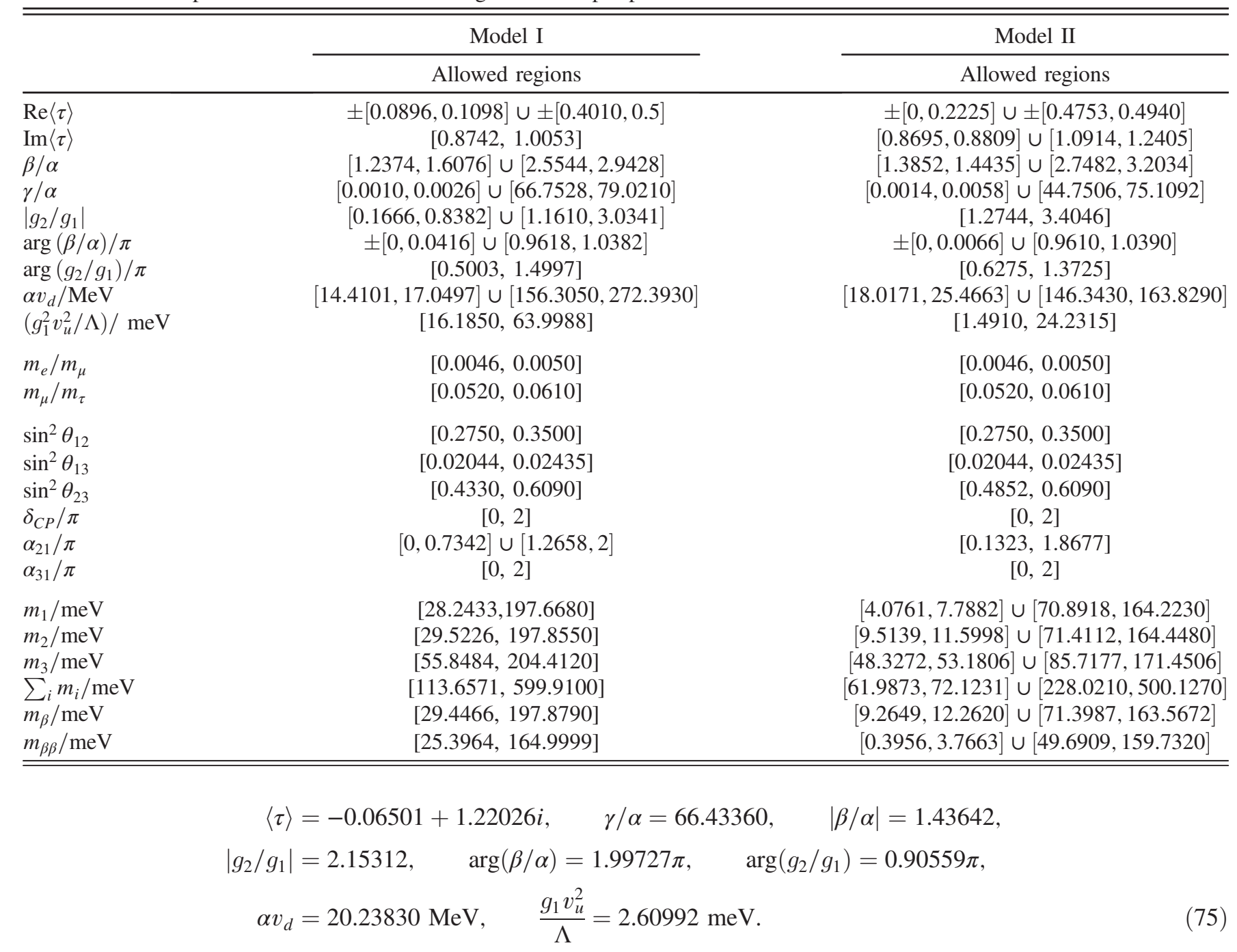

Accordingly the predictions for the lepton mixing parameters and neutrino masses are given by

$$
\begin{aligned}
\sin ^{2} \theta_{12} & =0.31001, \quad \sin ^{2} \theta_{13}=0.022370, \quad \sin ^{2} \theta_{23}=0.56298, \quad \delta_{C P}=1.5784 \pi \\
\alpha_{21} & =1.1235 \pi, \quad \alpha_{31}=0.4696 \pi, \quad m_{e} / m_{\mu}=0.00480 \mathrm{MeV}, \quad m_{\mu} / m_{\tau}=0.05649 \\
m_{1} & =4.3686 \mathrm{meV}, \quad m_{2}=9.6429 \mathrm{meV}, \quad m_{3}=50.4677 \mathrm{meV}, \\
m_{\beta} & =9.9010 \mathrm{meV}, \quad m_{\beta \beta}=2.9090 \mathrm{meV} .
\end{aligned}
$$

which are compatible with the experimental data at $1 \sigma$ level [71]. Notice that the neutrino masses are hierarchical normal ordering, the Planck bound $\sum_{i} m_{i}<120 \mathrm{meV}$ is satisfied, and $m_{\beta}$ and $m_{\beta \beta}$ are quite tiny. Analogous to what we have done for Model I, we scan over the parameter space of the model. The correlations between the input parameters and observables are shown in Fig. 2, and their allowed regions are given in Table IV. We notice that the $C P$ violating phases $\delta_{C P}, \alpha_{21}$ and $\alpha_{31}$ are strongly correlated with each other, and the phase $\arg (\beta / \alpha)$ is also close to 0 or $\pi$.

\section{Model III: Neutrino masses from seesaw mechanism with two right-handed neutrinos}

The neutrino masses are described by the minimal seesaw model with two right-handed neutrinos. We assign the left-handed leptons $L$ to a triplet 3 of $\tilde{S}_{4}$, only two righthanded neutrinos are introduced and they are assumed to transform as a doublet $\tilde{\mathbf{2}}^{\prime}$ under $\tilde{S}_{4}$, while the right-handed charged leptons $E_{1}^{c}, E_{2}^{c}$, and $E_{3}^{c}$ transform as singlets $\mathbf{1}, \mathbf{1}$, and $\hat{\mathbf{1}}$, respectively. We choose the modular weights of lepton fields are 

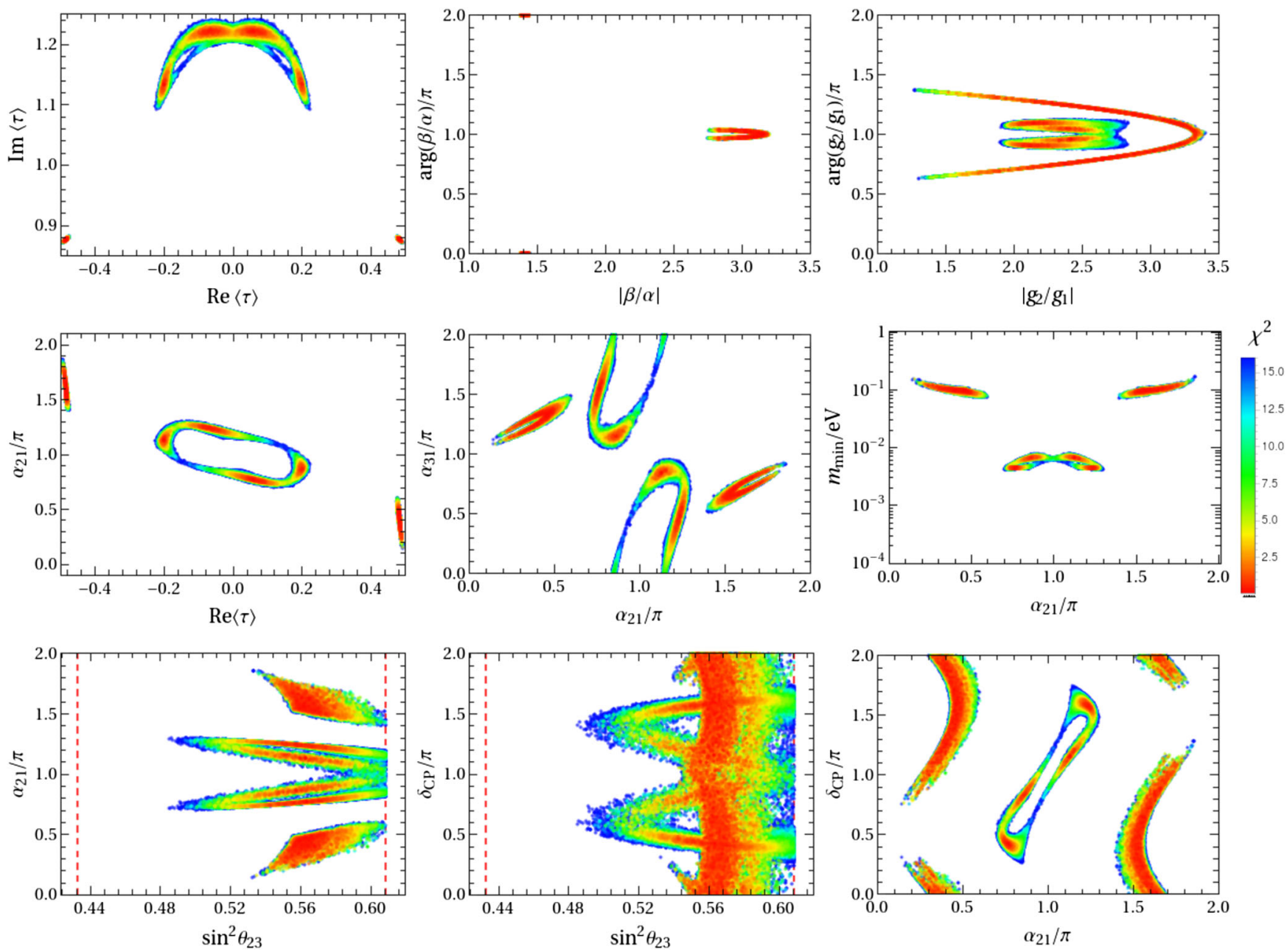

FIG. 2. The predicted correlations among the input free parameters, neutrino mixing angles and $C P$ violating phases in the Model II. The same convention as Fig. 1 is adopted.

$$
k_{L} / 2=1, \quad k_{N^{c}} / 2=\frac{3}{2}, \quad k_{E_{1}^{c}} / 2=1, \quad k_{E_{2}^{c}} / 2=3, \quad k_{E_{3}^{c}} / 2=4 .
$$

The masses of the charged leptons and neutrinos are described by the following superpotential,

$$
\begin{aligned}
& \mathcal{W}_{e}=\alpha\left(E_{1}^{c} L Y_{\mathbf{3}}^{(2)}\right)_{\mathbf{1}} H_{d}+\beta\left(E_{2}^{c} L Y_{\mathbf{3}}^{(4)}\right)_{\mathbf{1}} H_{d}+\gamma\left(E_{3}^{c} L Y_{\hat{\mathbf{z}}^{\prime} I}^{(5)}\right)_{\mathbf{1}} H_{d}+\delta\left(E_{3}^{c} L Y_{\hat{\mathbf{z}}^{\prime} I I}^{(5)}\right)_{\mathbf{1}} H_{d} \\
& \mathcal{W}_{\nu}=g\left(N^{c} L Y_{\mathbf{4}}^{\left(\frac{5}{2}\right)}\right)_{\mathbf{1}} H_{u}+\Lambda\left(N^{c} N^{c} Y_{\hat{\mathbf{z}}^{\prime}}^{(3)}\right)_{\mathbf{1}} .
\end{aligned}
$$

Notice that the term $\left(N^{c} N^{c} Y_{\hat{\mathbf{1}}^{\prime}}^{(3)}\right)_{\mathbf{1}}$ is allowed by symmetries of the model but gives a vanishing contribution because of the antisymmetric CG coefficient for the contraction $\tilde{\mathbf{2}}^{\prime} \otimes \tilde{\mathbf{2}}^{\prime} \rightarrow \hat{\mathbf{1}}$. We find the charged lepton and neutrino mass matrices are given by

$$
M_{e}=\left(\begin{array}{ccc}
\alpha Y_{\mathbf{3}, 1}^{(2)} & \alpha Y_{\mathbf{3}, 3}^{(2)} & \alpha Y_{\mathbf{3}, 2}^{(2)} \\
\beta Y_{\mathbf{3}, 1}^{(4)} & \beta Y_{\mathbf{3}, 3}^{(4)} & \beta Y_{\mathbf{3}, 2}^{(4)} \\
\gamma Y_{\hat{\mathbf{3}}^{\prime} I, 1}^{(5)}+\delta Y_{\hat{\mathbf{3}}^{\prime} I I, 1}^{(5)} & \gamma Y_{\hat{\mathbf{3}}^{\prime} I, 3}^{(5)}+\delta Y_{\hat{\mathbf{3}}^{\prime} I I, 3}^{(5)} & \gamma Y_{\hat{\mathbf{3}}^{\prime} I, 2}^{(5)}+\delta Y_{\hat{\mathbf{3}}^{\prime} I I, 2}^{(5)}
\end{array}\right) v_{d},
$$




$$
M_{N}=\left(\begin{array}{cc}
\sqrt{2} Y_{\hat{\mathbf{3}}^{\prime}, 2}^{(3)} & Y_{\hat{\mathbf{3}}^{\prime}, 1}^{(3)} \\
Y_{\hat{\mathbf{3}}^{\prime}, 1}^{(3)} & -\sqrt{2} Y_{\hat{\mathbf{3}}^{\prime}, 3}^{(3)}
\end{array}\right) \Lambda, \quad M_{D}=g\left(\begin{array}{ccc}
-\sqrt{2} Y_{\mathbf{4}, 4}^{\left(\frac{5}{2}\right)} & -Y_{\mathbf{4}, 2}^{\left(\frac{5}{2}\right)} & \sqrt{3} Y_{\mathbf{4}, 1}^{\left(\frac{5}{2}\right)} \\
\sqrt{2} Y_{\mathbf{4}, 2}^{\left(\frac{5}{2}\right)} & \sqrt{3} Y_{\mathbf{4}, 3}^{\left(\frac{5}{2}\right)} & -Y_{\mathbf{4}, 4}^{\left(\frac{5}{2}\right)}
\end{array}\right) v_{u}
$$

The parameters $\alpha, \beta$, and $\gamma$ are taken to be real and positive by rephasing right-handed charged lepton fields without loss of generality while the phase of $\delta$ cannot be removed. The light neutrino mass matrix only depends on the complex modulus $\tau$ besides the overall mass scale $g^{2} v_{u}^{2} / \Lambda$. Hence, this model has 8 real input parameters to describe the 11 observables including three charged lepton masses, three neutrino masses, three lepton mixing angles and two $C P$ violating phases. Notice that the modular invariant models typically use 8 independent parameters to describe the lepton sector [1]. We numerically scan over the parameter space, the parameters $\alpha, \beta, \gamma$, and $|\delta|$ are treated as random numbers between 0 and $10^{4}$, the phase $\arg (\delta)$ freely varies in the range of 0 and $2 \pi$, and the modulus $\tau$ is limited in the fundamental domain. The best fit values of the input parameters are determined to be

$$
\begin{gathered}
\langle\tau\rangle=0.07814+1.18349 i, \quad \beta / \alpha=65.95160, \quad \gamma / \alpha=752.95700, \\
|\delta| / \alpha=478.04500, \quad \arg (\delta)=0.03818 \pi, \quad \alpha v_{d}=1.4124 \mathrm{MeV}, \quad \frac{g v_{u}^{2}}{\Lambda}=22.03180 \mathrm{meV} .
\end{gathered}
$$

The lepton mixing parameters and neutrino masses are predicted to be
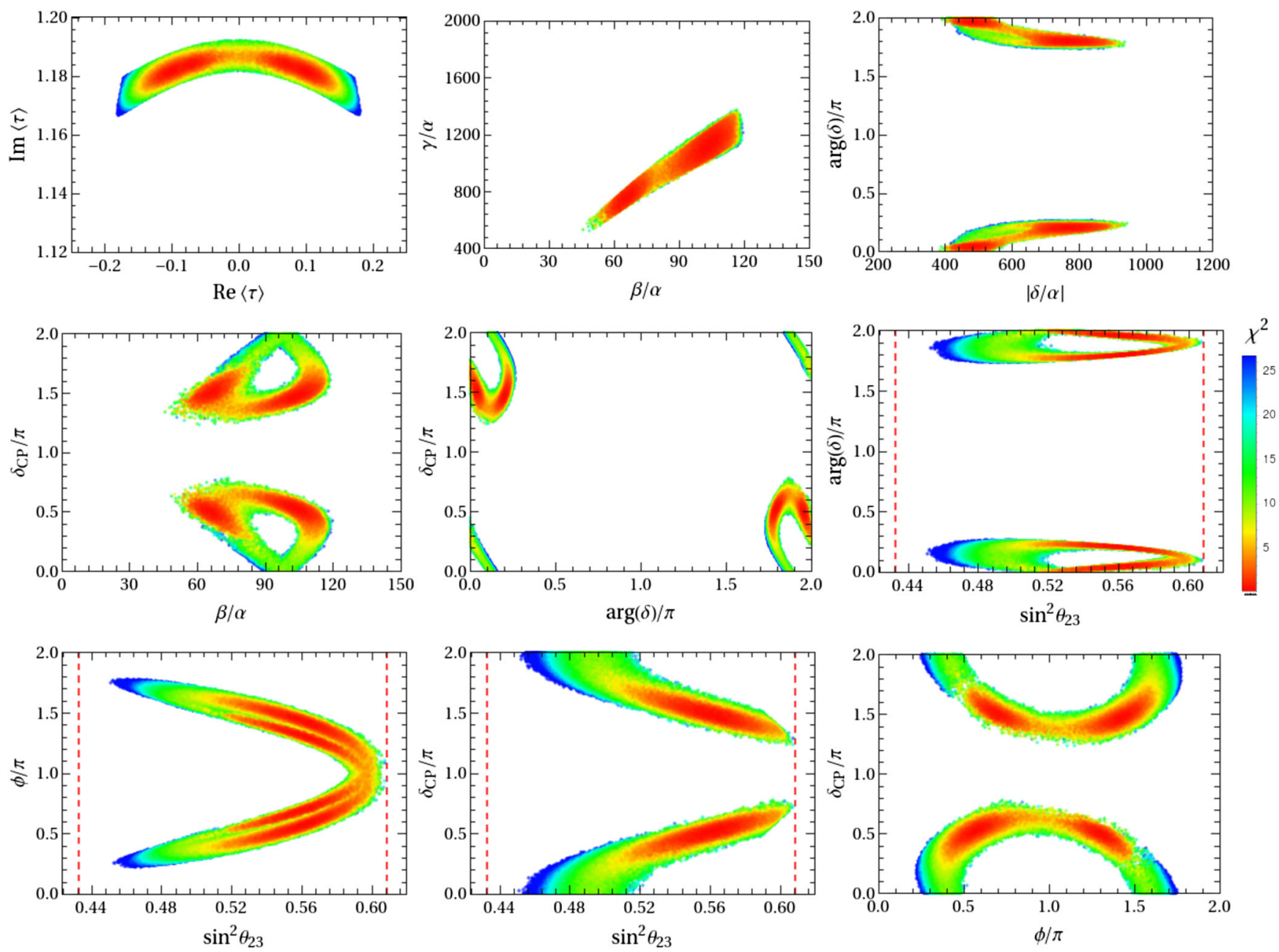

FIG. 3. The predicted correlations among the input free parameters, neutrino mixing angles and $C P$ violating phases in the Model III. The same convention as Fig. 1 is adopted. 
TABLE V. The predictions for the allowed ranges of the input parameters and observables in the Model III.

\begin{tabular}{lc}
\hline \hline Model III & Allowed regions \\
\hline $\operatorname{Re}\langle\tau\rangle$ & {$[-0.1808,0.1808]$} \\
$\operatorname{Im}\langle\tau\rangle$ & {$[1.1666,1.1921]$} \\
$\beta / \alpha$ & {$[45.8090,119.7920]$} \\
$\gamma / \alpha$ & {$[522.4570,1373.3900]$} \\
$\delta / \alpha$ & {$[385.5060,943.9640]$} \\
$\arg (\delta) / \pi$ & {$[0,0.2641] \cup[1.7359,2]$} \\
$\alpha v_{d} / \mathrm{meV}$ & {$[0.5502,1.5328]$} \\
$g^{2} v_{u}^{2} / \Lambda / \mathrm{meV}$ & {$[21.2934,22.6938]$} \\
$m_{e} / m_{\mu}$ & {$[0.0046,0.0050]$} \\
$m_{\mu} / m_{\tau}$ & {$[0.0520,0.0610]$} \\
$\sin ^{2} \theta_{12}$ & {$[0.2750,0.3500]$} \\
$\sin ^{2} \theta_{13}$ & {$[0.02044,0.02435]$} \\
$\sin ^{2} \theta_{23}$ & {$[0.4510,0.6076]$} \\
$\delta_{C P} / \pi$ & {$[0,0.7786] \cup[1.2214,2]$} \\
$\phi / \pi$ & {$[0.2235,1.7765]$} \\
$m_{1} / \mathrm{meV}$ & 0 \\
$m_{2} / \mathrm{meV}$ & {$[8.5965,8.5965]$} \\
$m_{3} / \mathrm{meV}$ & {$[48.1475,52.7218]$} \\
$\sum_{i} m_{i} / \mathrm{meV}$ & {$[56.7440,61.3183]$} \\
$m_{\beta} / \mathrm{meV}$ & {$[8.2293,9.6092]$} \\
$m_{\beta \beta} / \mathrm{meV}$ & {$[1.0896,4.1691]$} \\
\hline \hline
\end{tabular}

$$
\begin{array}{rlrl}
\sin ^{2} \theta_{12} & =0.31068, & \sin ^{2} \theta_{13}=0.022368, \\
\sin ^{2} \theta_{23} & =0.55700, & & \delta_{C P}=1.5178 \pi, \\
\phi & =0.6735 \pi, & & m_{e} / m_{\mu}=0.00480, \\
m_{\mu} / m_{\tau} & =0.05652, & m_{1}=0 \mathrm{meV}, \\
m_{2} & =8.5965 \mathrm{meV}, & m_{3}=50.2797 \mathrm{meV}, \\
m_{\beta} & =8.8878 \mathrm{meV}, & m_{\beta \beta}=3.4150 \mathrm{meV},
\end{array}
$$

which are in the experimentally preferred $1 \sigma$ range [71], and both effective neutrino masses $m_{\beta}$ and $m_{\beta \beta}$ are far below the sensitivity of forthcoming experiments. Notice that the lightest neutrino is always massless with $m_{1}=0$ because only two right-handed neutrinos are introduced. Consequently, the bound on neutrino mass sum from Planck is fulfilled. Moreover, the correlations between the input parameters and observables are shown in Fig. 3. We find that the mixing angle $\theta_{23}$ and the Dirac $C P$ violation phase $\delta_{C P}$ and the Majorana phase $\phi$ are strongly correlated with each other, and the values of $\delta_{C P}$ around $\pm 0.5 \pi$ is preferred. Finally we summarize the allowed ranges of the input parameters and observables in the Table V.

\section{SUMMARY AND CONCLUSIONS}

In the present work, we have extended the modular invariance approach [1] to include the half-integral weight modular forms. It is highly nontrivial to generalize integral weight modular forms to the nonintegral case, and a multiplier system is generally necessary for the consistency definition of nonintegral weight modular forms. In order to discuss the action of the full modular group on the halfintegral modular forms, one should extend the modular group $\mathrm{SL}_{2}(\mathbb{Z})$ to the metaplectic group $\mathrm{Mp}_{2}(\mathbb{Z})$ which is the double covering of $\mathrm{SL}_{2}(\mathbb{Z})$. As a result, we need to generalize the framework of modular invariant theory to the metaplectic modular invariant theory. Each modular multiplet is specified by its modular weight and the transformation under the finite metaplectic group. We show that the half-integral weight modular forms for the congruence subgroup $\Gamma(4 N)$ can be arranged into irreducible multiplets of finite metaplectic group $\tilde{\Gamma}_{4 N}$, which is the double covering of the homogeneous finite modular group $\Gamma_{4 N}^{\prime}$. We have considered the simplest case of level $4 N=4$ in the context of metaplectic modular invariance approach. The half-integral weight modular forms up to weight 6 are constructed in terms of the Jacobi theta constants, and they are decomposed into different irreducible multiplets of $\tilde{\Gamma}_{4}$. It is notable that the odd integral weight modular forms are in the representations $\hat{\mathbf{1}}, \hat{\mathbf{1}}^{\prime}, \mathbf{2}^{\prime}, \hat{\mathbf{3}}$, and $\hat{\mathbf{3}}^{\prime}$, the even integral weight modular forms are in the representations $\mathbf{1}, \mathbf{1}^{\prime}, \mathbf{2}, \mathbf{3}$, and $\mathbf{3}^{\prime}$, while the modular forms of weight $n+1 / 2$ with $n$ a generic non-negative integer are in the representations $\hat{\mathbf{2}}, \hat{\mathbf{2}}^{\prime}$, $\tilde{\mathbf{2}}, \tilde{\mathbf{2}}^{\prime}, \mathbf{4}$, and $\mathbf{4}^{\prime}$. It is worth noting that in the top-down approach from string theory, the wave functions of zero modes and massive modes on the magnetized torus behave as modular forms of weight $1 / 2$ [2]. It has been shown that the homogeneous finite modular group $\Gamma_{3}^{\prime} \cong T^{\prime}$ can naturally appear in string construction [47,51], and as shown in Ref. [6], in this case matter fields have fractional modular weights. However, it is still not known how to generate the metaplectic finite modular group $\tilde{\Gamma}_{4 N}$ together with the halfintegral weight modular forms in the top-down constructions. The connections between this bottom-up approach and the top-down string construction deserve more studies.

We present three typical models based on the finite metaplectic group $\tilde{\Gamma}_{4} \equiv \tilde{S}_{4}$. The neutrino masses are described by the effective Weinberg operator in the Model I, and neutrino masses arise from type I seesaw mechanism in Model II and Model III, and three righthanded neutrinos and two right-handed neutrinos are introduced in Model II and Model III respectively. The structure of these models is rather simple, and there are no additional flavons except the complex modulus $\tau$. The halfintegral weight modular forms are involved in either neutrino Yukawa couplings or charged lepton Yukawa couplings. Each model is analyzed numerically, the predictions are in excellent agreement with the experimental data on neutrino oscillation, beta decay, neutrinoless double decay and cosmology. Some of these predictions could be tested at forthcoming experiments. Finally, we perform a 
comprehensive numerical scan over the parameter space of the three models, some interesting correlations between free parameters and observables are shown in Figs. 1-3, the allowed regions of the input parameters and observable quantities are summarized in Tables IV and V. Our models use 8 (Model III) or 9 (Model I and Model II) free real parameters to describe 12 observables, consequently, they are very predictive. All these models can accommodate the experimental data very well for certain values of the free parameters. However, in common with all papers published in this field so far, it is impossible to dynamically fix the values of the few input parameters without further theoretical input. For example, it has been conjectured that the modulus $\tau$ could be fixed on the imaginary axis or along the border of the fundamental domain in modular invariant $\mathcal{N}=1$ supergravity theories $[23,76]$. Since the $\tilde{S}_{4}$ modular symmetry can describe the lepton sector very well, it is interesting to apply $\tilde{S}_{4}$ to explain the hierarchical quark masses and CKM mixing matrix.

In a similar fashion, other rational weight modular forms can be studied, and one needs to determine the corresponding metaplectic covering group to remove the ambiguity of multi-valued branches induced by rational powers. It is very lucky that the rational weight modular forms for the principal congruence subgroup of level odd $N \geq 5$ have been constructed by mathematicians [60,61], as summarized in Table I. There are no conceptual and mathematical obstacles to perform an analysis similar to the present work, although the group order of the corresponding metaplectic finite group is large. It is promising that the real weight modular forms can also be discussed in an analogous manner [63]. It is fascinating to use the simplest nontrivial case of real weight modular forms to understand the standard model flavor puzzle in future.

It is known that the Kähler potential is not completely fixed by the modular symmetry [4], nevertheless it could be strongly constrained in the top-down approach combining the modular flavor symmetry with traditional flavor symmetry [5,6,47,51]. The Kähler potential is also less constrained the metaplectic modular symmetry. We expect that the Kähler potential as well as the structure of the model should also be severely restricted if the metaplectic flavor symmetry is combined with traditional flavor symmetry. Moreover, it is well established that the $C P$ transformation consistent with modular group $\mathrm{SL}_{2}(\mathbb{Z})$ is uniquely $\tau \rightarrow-\tau^{*}$ up to modular transformations [46-50], and $C P$ is conserved if the value of modulus $\tau$ is pure imaginary or at the border of the fundamental domain. The modular group $\mathrm{SL}_{2}(\mathbb{Z})$ is extended to the metaplectic group $\mathrm{Mp}_{2}(\mathbb{Z})$ in this work. It is interesting to investigate the $C P$ transformation consistent with $\mathrm{Mp}_{2}(\mathbb{Z})$, the $C P$ conserved values of $\tau$ and the implications for modular models. All these are left for future projects. We conclude that halfintegral weight modular forms as well as more general rational weight modular forms provide new opportunities and possibilities for modular model building, and there are many relevant aspects which deserve studying further.

In conclusion, new flavor symmetry groups emerge when one considers the half-integral or more general rational weight modular forms in the modular invariance approach. The formalism we present opens up the door to build modular invariant flavor models with rational weight modular forms. Although the benchmark models which we have constructed with half-integral weight modular forms are no more or less predictive than other models based on integral weight modular forms with minimal number of free parameters, they provide new possibilities which are different from those of other models leading to new predictions.

\section{ACKNOWLEDGEMENTS}

X. G. L., B. Y. Q., and G J D are supported by the National Natural Science Foundation of China under Grants No. 11975224, No. 11835013, and No. 11947301. C. Y. Y. is supported in part by the Grants No. NSFC-11975130, No. NSFC-12035008, No. NSFC-12047533, by the National Key Research and Development Program of China under Grant No. 2017YFA0402200 and the China Post-doctoral Science Foundation under Grant No. 2018M641621.

\section{APPENDIX A: MULTIPLIER SYSTEM OF RATIONAL WEIGHT MODULAR FORMS}

As a general principle, when we discuss the general nonintegral weight $r$ modular forms, it is necessary to introduce the so-called multiplier system $v(\gamma)$ to ensure the existence of well-defined automorphy factors $j_{r}(\gamma, \tau)=$ $v(\gamma)(c \tau+d)^{r}$, namely $j_{r}\left(\gamma_{1} \gamma_{2}, \tau\right)=j_{r}\left(\gamma_{1}, \gamma_{2} \tau\right) j_{r}\left(\gamma_{2}, \tau\right)$ for any $\gamma_{1}, \gamma_{2} \in \Gamma^{\prime}$, where $\Gamma^{\prime}$ is a subgroup of $\mathrm{SL}_{2}(\mathbb{Z})$. Thus the definition of the modular form of nonintegral weight $r$ for the subgroup $\Gamma^{\prime}$ is

$$
f(\gamma \tau)=v(\gamma)(c \tau+d)^{r} f(\tau), \quad \gamma \in \Gamma^{\prime} .
$$

The multiplier system $v(\gamma)$ heavily depends on $\Gamma^{\prime}$. For principal congruence subgroup $\Gamma(4 N)$, the multiplier system is the Kronecker symbol mentioned above, it is used to define the half-integral weight modular forms for $\Gamma(4 N)$. For other principal congruence subgroup $\Gamma(N)$ of level odd integer $N \geq 5$, a unified construction of multiplier systems denote by $v_{N}(\gamma)$ is given in [60], and the corresponding modular forms are of weight $(N-3) /(2 N)$. as already mentioned in the Sec. II C. Specifically, $v_{N}$ is given by the following formula 


$$
v_{N}(\gamma)= \begin{cases}1 & \text { if } c=0 \\ \exp \left(-2 \pi i \frac{3 \operatorname{sign}(c)\left(N^{2}-1\right)}{8 N}\right) \exp \left(2 \pi i \frac{N^{2}-1}{8 N} \Phi(\gamma)\right) & \text { if } c \neq 0\end{cases}
$$

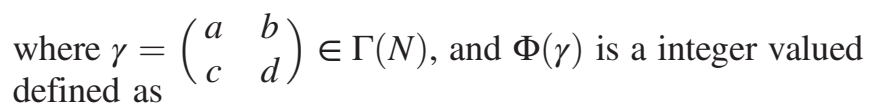

$$
\Phi(\gamma)= \begin{cases}\frac{b}{d} & \text { if } c=0 \\ \frac{a+d}{c}-12 \operatorname{sign}(c) s(d,|c|) & \text { if } c \neq 0\end{cases}
$$

where $s(d,|c|)$ is the Dedekind sum with

$$
s(h, k)=\sum_{\mu=1}^{k}\left(\left(\frac{h \mu}{k}\right)\right)\left(\left(\frac{\mu}{k}\right)\right),
$$

for integers $h, k(k \neq 0)$. Here $((x))$ is the sawtooth function defined by

$$
((x))= \begin{cases}x-[x]-\frac{1}{2} & \text { if } x \notin \mathbb{Z}, \\ 0 & \text { if } x \in \mathbb{Z},\end{cases}
$$

with $[x]$ the floor function. Note that the multiplier system $v_{N}(\gamma)$ is an $N$-th root of unity, consequently, $v_{N}(\gamma)^{N}=1$ for all $\gamma \in \Gamma(N)$. In short, $v_{N}(\gamma)(c \tau+d)^{(N-3) / 2 N}$ is the automorphy factor for the modular form of weight $(N-3) /(2 N)$ at level odd integer $N \geq 5$.

\section{APPENDIX B: GROUP THEORY OF $\tilde{S}_{4}$}

The group $\tilde{S}_{4}$ has 96 elements, and it can be generated by three generators $\tilde{S}, \tilde{T}$ and $\tilde{R}$ obeying the rules:

$$
\begin{aligned}
\tilde{S}^{2} & =\tilde{R}, & (\tilde{S} \tilde{T})^{3}=\tilde{T}^{4}=\tilde{R}^{4}=1, \\
\tilde{S} \tilde{R} & =\tilde{R} \tilde{S}, & \tilde{T} \tilde{R}=\tilde{R} \tilde{T} .
\end{aligned}
$$

After we input these multiplication rules in GAP [59], its group ID can be determined as $[96,67]$. Notice that $S_{4}$ is not a subgroup of $\tilde{S}_{4}$, it is isomorphic to the quotient group of $\tilde{S}_{4}$ over $Z_{4}^{\tilde{R}}$, i.e., $S_{4} \cong \tilde{S}_{4} / Z_{4}^{\tilde{R}}$, where $Z_{4}^{\tilde{R}}=\left\{1, \tilde{R}, \tilde{R}^{2}, \tilde{R}^{3}\right\}$ is the center and a normal subgroup of $\tilde{S}_{4}$. The finite metaplectic group $\tilde{S}_{4}$ is a quadruple cover of $S_{4}$ or double cover of $S_{4}^{\prime}$. It is notable that $\tilde{S}_{4}$ is isomorphic to the semidirect product of $T^{\prime}$ with $Z_{4}$, namely $\tilde{S}_{4} \cong T^{\prime} \rtimes Z_{4}$, where $T^{\prime} \cong \Gamma_{3}^{\prime}$ is the double covering of group $A_{4}$ [44]. Hence, $\tilde{S}_{4} \cong T^{\prime} \rtimes Z_{4}$ can also be expressed in terms of another set of generators $S, T$, and $R$ which satisfy the following rules:

$$
\begin{aligned}
& S^{4}=(S T)^{3}=T^{3}=1, \quad S^{2} T=T S^{2}, \\
& R^{4}=1, \quad R S R^{-1}=S, \quad R T R^{-1}=(S T)^{2} .
\end{aligned}
$$

The first two with generators $S$ and $T$ generate the group $T^{\prime}$, the third one with generator $R$ generates the cyclic group $Z_{4}$. The last two relations define the semidirect product “ $\rtimes$ ". The generators $\tilde{S}, \tilde{T}$, and $\tilde{R}$ can be expressed in terms of $S, T$, and $R$ or vice versa:

$$
\begin{cases}S=\tilde{T}^{2} \tilde{R}, & T=(\tilde{S} \tilde{T})^{2}, \quad R=\tilde{T}, \\ \tilde{S}=T^{2} R^{3}, & \tilde{T}=R,\end{cases}
$$

Therefore, $\tilde{S}_{4}$ can also be regarded as a split extension of $T^{\prime}$ by $Z_{4}$. Note that $Z_{4}$ here is different from $Z_{4}^{\tilde{R}}$ mentioned above. All the elements of $\tilde{S}_{4}$ group can be divided into 16 conjugacy classes:

$$
\begin{aligned}
& 1 C_{1}=\{1\} \\
& 1 C_{2}=\left\{\tilde{R}^{2}\right\}=\left(1 C_{1}\right) \cdot \tilde{R}^{2}, \\
& 6 C_{2}=\left\{\tilde{T}^{2}, \tilde{T}^{2} \tilde{R}^{2},\left(\tilde{S} \tilde{T}^{2}\right)^{2}, \tilde{S} \tilde{T}^{2} \tilde{S}^{3},\left(\tilde{S} \tilde{T}^{2}\right)^{2} \tilde{R}^{2}, \tilde{S} \tilde{T}^{2} \tilde{S}^{3} \tilde{R}^{2}\right\}, \\
& 8 C_{3}=\left\{\tilde{S} \tilde{T}, \tilde{T} \tilde{S},(\tilde{S} \tilde{T})^{2},(\tilde{T} \tilde{S})^{2}, \tilde{T}^{2} \tilde{S} \tilde{T}^{3}, \tilde{T}^{3} \tilde{S} \tilde{T}^{2}, \tilde{T}^{2} \tilde{S}^{3} \tilde{T} \tilde{R}^{2}, \tilde{T} \tilde{S}^{3} \tilde{T}^{2} \tilde{R}^{2}\right\}, \\
& 1 C_{4}=\{\tilde{R}\}=\left(1 C_{1}\right) \cdot \tilde{R}, \\
& 1 C_{4}^{\prime}=\left\{\tilde{R}^{3}\right\}=\left(1 C_{1}\right) \cdot \tilde{R}^{3}, \\
& 6 C_{4}=\left\{\tilde{T}, \tilde{T}^{3} \tilde{S}^{2}, \tilde{T}^{2} \tilde{S} \tilde{R}^{2}, \tilde{S} \tilde{T}^{2} \tilde{R}^{2}, \tilde{T} \tilde{S} \tilde{T} \tilde{R}^{2}, \tilde{S} \tilde{T} \tilde{S}^{3} \tilde{R}^{2}\right\}, \\
& 6 C_{4}^{\prime}=\left\{\tilde{T} \tilde{R}, \tilde{T}^{3} \tilde{S}^{2} \tilde{R}, \tilde{T}^{2} \tilde{S} \tilde{R}^{3}, \tilde{S} \tilde{T}^{2} \tilde{R} \tilde{R}^{3}, \tilde{T} \tilde{S} \tilde{T} \tilde{R}^{3}, \tilde{S} \tilde{T} \tilde{S}\right\}=\left(6 C_{4}\right) \cdot \tilde{R}, \\
& 6 C_{4}^{\prime \prime}=\left\{\tilde{T} \tilde{R}^{2}, \tilde{T}^{3} \tilde{S}^{2} \tilde{R}^{2}, \tilde{T}^{2} \tilde{S}, \tilde{S} \tilde{T}^{2}, \tilde{T} \tilde{S} \tilde{T}, \tilde{S} \tilde{T} \tilde{S}^{3}\right\}=\left(6 C_{4}\right) \cdot \tilde{R}^{2}, \\
& 6 C_{4}^{\prime \prime \prime}=\left\{\tilde{T} \tilde{R}^{3}, \tilde{T}^{3}, \tilde{T}^{2} \tilde{S} \tilde{R}, \tilde{S} \tilde{T}^{2} \tilde{R}, \tilde{T} \tilde{S} \tilde{T} \tilde{R}, \tilde{S} \tilde{T} \tilde{S}^{3} \tilde{R}\right\}=\left(6 C_{4}\right) \cdot \tilde{R}^{3},
\end{aligned}
$$


TABLE VI. Character table of $\tilde{S}_{4}$, and we give a representative element for each conjugacy class in the second row.

\begin{tabular}{lccccccccccccccccc}
\hline \hline Classes & $1 C_{1}$ & $1 C_{2}$ & $6 C_{2}$ & $8 C_{3}$ & $1 C_{4}$ & $1 C_{4}^{\prime}$ & $6 C_{4}$ & $6 C_{4}^{\prime}$ & $6 C_{4}^{\prime \prime}$ & $6 C_{4}^{\prime \prime \prime}$ & $6 C_{4}^{\prime \prime \prime}$ & $8 C_{6}$ & $12 C_{8}$ & $12 C_{8}^{\prime}$ & $8 C_{12}$ & $8 C_{12}^{\prime}$ \\
\hline$G$ & 1 & $\tilde{R}$ & $\tilde{T}^{2}$ & $\tilde{S} \tilde{T}$ & $\tilde{R}$ & $\tilde{R}^{3}$ & $\tilde{T}$ & $\tilde{T} \tilde{R}$ & $\tilde{T} \tilde{R}^{2}$ & $\tilde{T} \tilde{R}^{3}$ & $\tilde{T}^{2} \tilde{R}$ & $\tilde{S} \tilde{T} \tilde{R}^{2}$ & $\tilde{S}$ & $\tilde{S} \tilde{R}$ & $\tilde{S} \tilde{T} \tilde{R}$ & $\tilde{S} \tilde{T} \tilde{R}^{3}$ \\
\hline $\mathbf{1}$ & 1 & 1 & 1 & 1 & 1 & 1 & 1 & 1 & 1 & 1 & 1 & 1 & 1 & 1 & 1 & 1 \\
$\mathbf{1}^{\prime}$ & 1 & 1 & 1 & 1 & 1 & 1 & -1 & -1 & -1 & -1 & 1 & 1 & -1 & -1 & 1 & 1 \\
$\hat{\mathbf{1}}$ & 1 & 1 & -1 & 1 & -1 & -1 & $-i$ & $i$ & $-i$ & $i$ & 1 & 1 & $i$ & $-i$ & -1 & -1 \\
$\hat{\mathbf{1}}^{\prime}$ & 1 & 1 & -1 & 1 & -1 & -1 & $i$ & $-i$ & $i$ & $-i$ & 1 & 1 & $-i$ & $i$ & -1 & -1 \\
$\mathbf{2}$ & 2 & 2 & 2 & -1 & 2 & 2 & 0 & 0 & 0 & 0 & 2 & -1 & 0 & 0 & -1 & -1 \\
$\mathbf{2}^{\prime}$ & 2 & 2 & -2 & -1 & -2 & -2 & 0 & 0 & 0 & 0 & 2 & -1 & 0 & 0 & 1 & 1 \\
$\hat{\mathbf{2}}$ & 2 & -2 & 0 & -1 & $2 i$ & $-2 i$ & $1+i$ & $-1+i$ & $-1-i$ & $1-i$ & 0 & 1 & 0 & 0 & $-i$ & $i$ \\
$\hat{\mathbf{2}}^{\prime}$ & 2 & -2 & 0 & -1 & $2 i$ & $-2 i$ & $-1-i$ & $1-i$ & $1+i$ & $-1+i$ & 0 & 1 & 0 & 0 & $-i$ & $i$ \\
$\tilde{\mathbf{2}}^{\prime}$ & 2 & -2 & 0 & -1 & $-2 i$ & $2 i$ & $1-i$ & $-1-i$ & $-1+i$ & $1+i$ & 0 & 1 & 0 & 0 & $i$ & $-i$ \\
$\tilde{\mathbf{2}}^{\prime}$ & 2 & -2 & 0 & -1 & $-2 i$ & $2 i$ & $-1+i$ & $1+i$ & $1-i$ & $-1-i$ & 0 & 1 & 0 & 0 & $i$ & $-i$ \\
$\mathbf{3}$ & 3 & 3 & -1 & 0 & 3 & 3 & 1 & 1 & 1 & 1 & -1 & 0 & -1 & -1 & 0 & 0 \\
$\mathbf{3}^{\prime}$ & 3 & 3 & -1 & 0 & 3 & 3 & -1 & -1 & -1 & -1 & -1 & 0 & 1 & 1 & 0 & 0 \\
$\hat{\mathbf{3}}_{\hat{\mathbf{3}}^{\prime}}^{3}$ & 3 & 1 & 0 & -3 & -3 & $-i$ & $i$ & $-i$ & $i$ & -1 & 0 & $-i$ & $i$ & 0 & 0 \\
$\mathbf{4}$ & 3 & 3 & 1 & 0 & -3 & -3 & $i$ & $-i$ & $i$ & $-i$ & -1 & 0 & $i$ & $-i$ & 0 & 0 \\
$\mathbf{4}^{\prime}$ & 4 & -4 & 0 & 1 & $4 i$ & $-4 i$ & 0 & 0 & 0 & 0 & 0 & -1 & 0 & 0 & $i$ & $-i$ \\
\hline \hline
\end{tabular}

$$
\begin{aligned}
6 C_{4}^{\prime \prime \prime}= & \left\{\tilde{T}^{2} \tilde{R}, \tilde{T}^{2} \tilde{R}^{3},\left(\tilde{S} \tilde{T}^{2}\right)^{2} \tilde{R}, \tilde{S} \tilde{T}^{2} \tilde{S}^{3} \tilde{R},\left(\tilde{S} \tilde{T}^{2}\right)^{2} \tilde{R}^{3}, \tilde{S} \tilde{T}^{2} \tilde{S}\right\}=\left(6 C_{2}\right) \cdot \tilde{R}, \\
8 C_{6}= & \left\{\tilde{S} \tilde{T} \tilde{R}^{2}, \tilde{T} \tilde{S} \tilde{R}^{2},(\tilde{S} \tilde{T})^{2} \tilde{R}^{2},(\tilde{T} \tilde{S})^{2} \tilde{R}^{2}, \tilde{T}^{2} \tilde{S} \tilde{T}^{3} \tilde{R}^{2}, \tilde{T}^{3} \tilde{S} \tilde{T}^{2} \tilde{R}^{2},\right. \\
& \left.\tilde{T}^{2} \tilde{S}^{3} \tilde{T}, \tilde{T} \tilde{S}^{3} \tilde{T}^{2}\right\}=\left(8 C_{3}\right) \cdot \tilde{R}^{2}, \\
12 C_{8}= & \left\{\tilde{S}, \tilde{S} \tilde{R}^{2}, \tilde{T}^{2} \tilde{S} \tilde{T}^{2}, \tilde{T}^{3} \tilde{S} \tilde{T}, \tilde{T} \tilde{S}^{3}, \tilde{T}^{2} \tilde{S} \tilde{T}^{2} \tilde{R}^{2}, \tilde{T}^{3} \tilde{S} \tilde{T}^{2} \tilde{R}^{2}, \tilde{S} \tilde{T}^{2} \tilde{S}^{3} \tilde{T}, \tilde{T} \tilde{S} \tilde{T}^{2} \tilde{S}^{3},\right. \\
& \left.\tilde{T} \tilde{S} \tilde{T}^{3} \tilde{R}^{2}, \tilde{S} \tilde{T}^{2} \tilde{S}^{3} \tilde{T} \tilde{R}^{2}, \tilde{T} \tilde{S} \tilde{T}^{2} \tilde{S}^{3} \tilde{R}^{2}\right\} \\
12 C_{8}^{\prime}= & \left\{\tilde{S} \tilde{R}, \tilde{S} \tilde{R}^{3}, \tilde{T}^{2} \tilde{S} \tilde{T}^{2} \tilde{R}, \tilde{T}^{3} \tilde{S} \tilde{T} \tilde{R}, \tilde{T} \tilde{S} \tilde{T}^{3} \tilde{R}, \tilde{T}^{2} \tilde{S} \tilde{T}^{2} \tilde{R}^{3}, \tilde{T}^{3} \tilde{S} \tilde{T} \tilde{R}^{3}, \tilde{S} \tilde{T}^{2} \tilde{S}^{3} \tilde{T} \tilde{R},\right. \\
& \left.\tilde{T} \tilde{S} \tilde{T}^{2} \tilde{S}^{3} \tilde{R}, \tilde{T} \tilde{S} \tilde{T}^{3} \tilde{R}^{3}, \tilde{S} \tilde{T}^{2} \tilde{S}^{3} \tilde{T} \tilde{R}^{3}, \tilde{T} \tilde{S} \tilde{T}^{2} \tilde{S}\right\}=\left(12 C_{8}\right) \cdot \tilde{R}, \\
8 C_{12}= & \left\{\tilde{S} \tilde{T} \tilde{R}, \tilde{T} \tilde{S} \tilde{R},(\tilde{S} \tilde{T})^{2} \tilde{R},(\tilde{T} \tilde{S})^{2} \tilde{R}, \tilde{T}^{2} \tilde{S} \tilde{T}^{3} \tilde{R}, \tilde{T}^{3} \tilde{S} \tilde{T}^{2} \tilde{R}, \tilde{T}^{2} \tilde{S}^{3} \tilde{T} \tilde{R}^{3},\right. \\
& \tilde{T} \tilde{S}^{3} \tilde{T}^{2} \tilde{R} 3=\left(8 C_{3}\right) \cdot \tilde{R}, \\
8 C_{12}^{\prime}= & \left\{\tilde{S} \tilde{T} \tilde{R}^{3}, \tilde{T} \tilde{S} \tilde{R}^{3},(\tilde{S} \tilde{T})^{2} \tilde{R}^{3},(\tilde{T} \tilde{S})^{2} \tilde{R}^{3}, \tilde{T}^{2} \tilde{S} \tilde{T}^{3} \tilde{R}^{3}, \tilde{T}^{3} \tilde{S} \tilde{T}^{2} \tilde{R}^{3},\right. \\
& \left.\tilde{T}^{2} \tilde{S}^{3} \tilde{T} \tilde{R}, \tilde{T} \tilde{S}^{3} \tilde{T}^{2} \tilde{R}\right\}=\left(8 C_{3}\right) \cdot \tilde{R}^{3} .
\end{aligned}
$$

where $k C_{n}$ denotes a conjugacy class with $k$ elements of order $n$. Note that some of these conjugacy classes can be written as the product of the others with $\tilde{R}, \tilde{R}^{2}$, or $\tilde{R}^{3}$. There are four one-dimensional irreducible representations $\mathbf{1}, \mathbf{1}^{\prime}, \hat{\mathbf{1}}$, and $\hat{\mathbf{1}}^{\prime}$, six two-dimensional irreducible representations $\mathbf{2}, \mathbf{2}^{\prime}, \hat{\mathbf{2}}, \hat{\mathbf{2}}^{\prime}, \tilde{\mathbf{2}}$, and $\tilde{\mathbf{2}}^{\prime}$, four three-dimensional irreducible representations $\mathbf{3}, \mathbf{3}^{\prime}, \hat{\mathbf{3}}$, and $\hat{\mathbf{3}}^{\prime}$, and two four-dimensional irreducible representations $\mathbf{4}, \mathbf{4}^{\prime}$. We have summarized the explicit matrix representations in Table VII. In the representations $\mathbf{1}, \mathbf{1}^{\prime}, \mathbf{2}, \mathbf{3}$ and $\mathbf{3}^{\prime}$, the generator $\tilde{R}=1$ is an identity matrix, the representation matrices of $\tilde{S}$ and $\tilde{T}$ coincide with those of $S_{4}$. Consequently, $\tilde{S}_{4}$ can not be distinguished from $S_{4}$ in these representations since they are represented by the same set of matrices. In the representations $\hat{\mathbf{1}}, \hat{\mathbf{1}}^{\prime}, \mathbf{2}^{\prime}, \hat{\mathbf{3}}$, and $\hat{\mathbf{3}}^{\prime}$, the generator $\tilde{R}=-1$. The character table of $\tilde{S}_{4}$ can be obtained by taking the trace of the representation matrices of the representative elements, and it is shown in table VI. Moreover, the Kronecker products between all irreducible representations are given as follows: 
TABLE VII. The representation matrices of the generators $\tilde{S}, \tilde{T}$ and $\tilde{R}$ for different irreducible representations of $\tilde{S}_{4}$ in the $\tilde{T}$-diagonal basis.

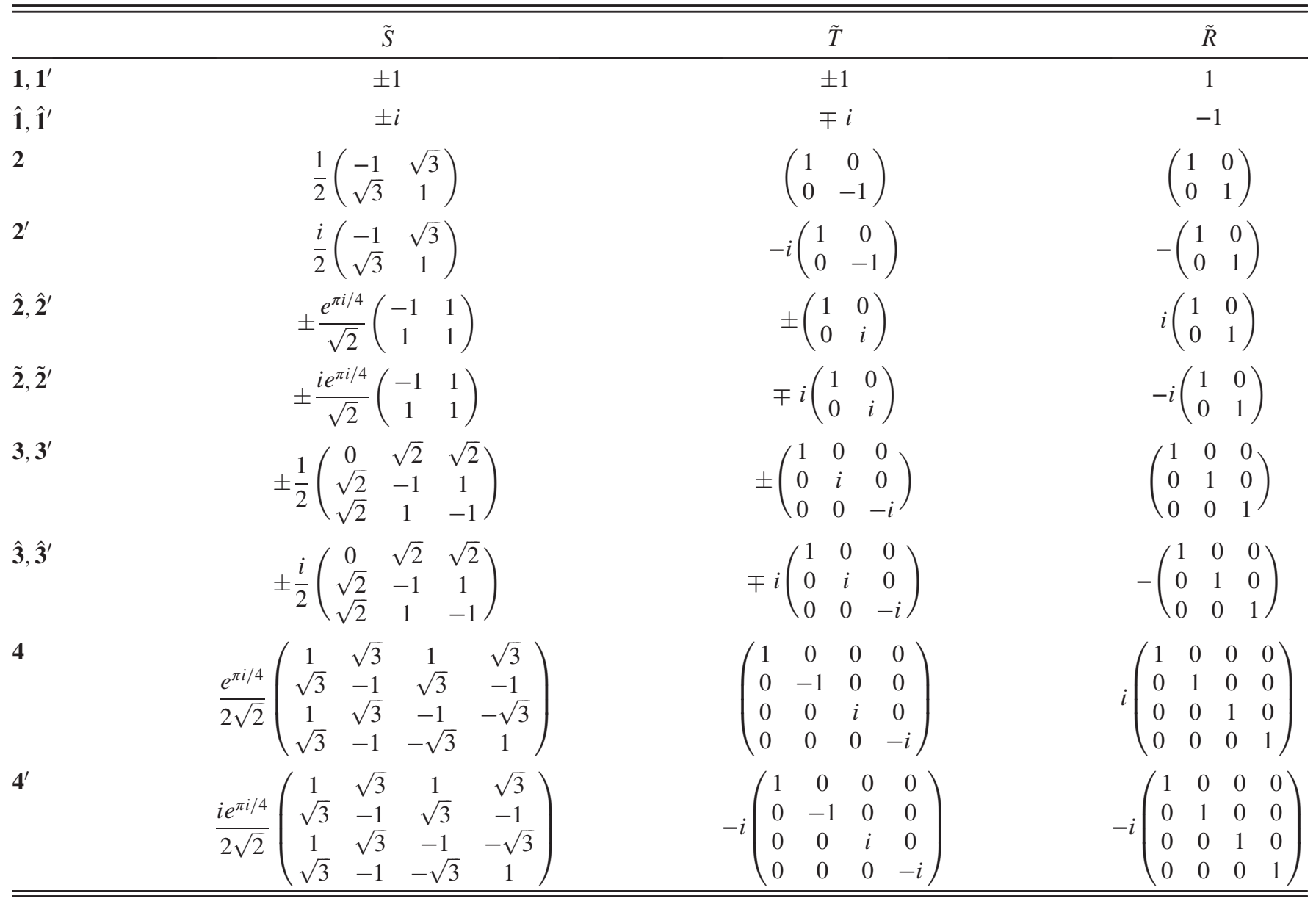

$\mathbf{1} \otimes \mathbf{1}=\mathbf{1}^{\prime} \otimes \mathbf{1}^{\prime}=\hat{\mathbf{1}} \otimes \hat{\mathbf{1}}^{\prime}=\mathbf{1}, \quad \mathbf{1} \otimes \mathbf{1}^{\prime}=\hat{\mathbf{1}} \otimes \hat{\mathbf{1}}=\hat{\mathbf{1}}^{\prime} \otimes \hat{\mathbf{1}}^{\prime}=\mathbf{1}^{\prime}$,

$1 \otimes \hat{\mathbf{1}}=\mathbf{1}^{\prime} \otimes \hat{\mathbf{1}}^{\prime}=\hat{\mathbf{1}}, \quad \mathbf{1} \otimes \hat{\mathbf{1}}^{\prime}=\mathbf{1}^{\prime} \otimes \hat{\mathbf{1}}=\hat{\mathbf{1}}^{\prime}$,

$1 \otimes 2=1^{\prime} \otimes 2=\hat{1} \otimes 2^{\prime}=\hat{1}^{\prime} \otimes 2^{\prime}=2, \quad 1 \otimes 2^{\prime}=1^{\prime} \otimes 2^{\prime}=\hat{1} \otimes 2=\hat{1}^{\prime} \otimes 2=2^{\prime}$,

$\mathbf{1} \otimes \hat{\mathbf{2}}=\mathbf{1}^{\prime} \otimes \hat{\mathbf{2}}^{\prime}=\hat{\mathbf{1}} \otimes \tilde{\mathbf{2}}^{\prime}=\hat{\mathbf{1}}^{\prime} \otimes \tilde{\mathbf{2}}=\hat{\mathbf{2}}, \quad \mathbf{1} \otimes \hat{\mathbf{2}}^{\prime}=\mathbf{1}^{\prime} \otimes \hat{\mathbf{2}}=\hat{\mathbf{1}} \otimes \tilde{\mathbf{2}}=\hat{\mathbf{1}}^{\prime} \otimes \tilde{\mathbf{2}}^{\prime}=\hat{\mathbf{2}}^{\prime}$,

$\mathbf{1} \otimes \tilde{\mathbf{2}}=\mathbf{1}^{\prime} \otimes \tilde{\mathbf{2}}^{\prime}=\hat{\mathbf{1}} \otimes \hat{\mathbf{2}}=\hat{\mathbf{1}}^{\prime} \otimes \hat{\mathbf{2}}^{\prime}=\tilde{\mathbf{2}}, \quad \mathbf{1} \otimes \tilde{\mathbf{2}}^{\prime}=\mathbf{1}^{\prime} \otimes \tilde{\mathbf{2}}=\hat{\mathbf{1}} \otimes \hat{\mathbf{2}}^{\prime}=\hat{\mathbf{1}}^{\prime} \otimes \hat{\mathbf{2}}=\tilde{\mathbf{2}}^{\prime}$,

$\mathbf{1} \otimes \mathbf{3}=\mathbf{1}^{\prime} \otimes \mathbf{3}^{\prime}=\hat{\mathbf{1}} \otimes \hat{\mathbf{3}}^{\prime}=\hat{\mathbf{1}}^{\prime} \otimes \hat{\mathbf{3}}=\mathbf{3}, \quad \mathbf{1} \otimes \mathbf{3}^{\prime}=\mathbf{1}^{\prime} \otimes \mathbf{3}=\hat{\mathbf{1}} \otimes \hat{\mathbf{3}}=\hat{\mathbf{1}}^{\prime} \otimes \hat{\mathbf{3}}^{\prime}=\mathbf{3}^{\prime}$,

$\mathbf{1} \otimes \hat{\mathbf{3}}=\mathbf{1}^{\prime} \otimes \hat{\mathbf{3}}^{\prime}=\hat{\mathbf{1}} \otimes \mathbf{3}=\hat{\mathbf{1}}^{\prime} \otimes \mathbf{3}^{\prime}=\hat{\mathbf{3}}, \quad \mathbf{1} \otimes \hat{\mathbf{3}}^{\prime}=\mathbf{1}^{\prime} \otimes \hat{\mathbf{3}}=\hat{\mathbf{1}} \otimes \mathbf{3}^{\prime}=\hat{\mathbf{1}}^{\prime} \otimes \mathbf{3}=\hat{\mathbf{3}}^{\prime}$,

$1 \otimes 4=1^{\prime} \otimes 4=\hat{1} \otimes 4^{\prime}=\hat{\mathbf{1}}^{\prime} \otimes 4^{\prime}=\mathbf{2} \otimes \hat{\mathbf{2}}=2 \otimes \hat{\mathbf{2}}^{\prime}=\mathbf{2}^{\prime} \otimes \tilde{\mathbf{2}}=\mathbf{2}^{\prime} \otimes \tilde{\mathbf{2}}^{\prime}=4$,

$1 \otimes 4^{\prime}=1^{\prime} \otimes 4^{\prime}=\hat{1} \otimes 4=\hat{1}^{\prime} \otimes 4=2 \otimes \tilde{2}=2 \otimes \tilde{2}^{\prime}=2^{\prime} \otimes \hat{2}=2^{\prime} \otimes \hat{2}^{\prime}=4^{\prime}$,

$2 \otimes 2=2^{\prime} \otimes 2^{\prime}=1 \oplus 1^{\prime} \oplus 2, \quad 2 \otimes 2^{\prime}=\hat{1} \oplus \hat{1}^{\prime} \oplus 2^{\prime}$,

$\hat{\mathbf{2}} \otimes \hat{\mathbf{2}}=\hat{\mathbf{2}}^{\prime} \otimes \hat{\mathbf{2}}^{\prime}=\tilde{\mathbf{2}} \otimes \tilde{\mathbf{2}}^{\prime}=\hat{\mathbf{1}}^{\prime} \oplus \hat{\mathbf{3}}^{\prime}, \quad \hat{\mathbf{2}} \otimes \hat{\mathbf{2}}^{\prime}=\tilde{\mathbf{2}} \otimes \tilde{\mathbf{2}}=\tilde{\mathbf{2}}^{\prime} \otimes \tilde{\mathbf{2}}^{\prime}=\hat{\mathbf{1}} \oplus \hat{\mathbf{3}}$,

$\hat{\mathbf{2}} \otimes \tilde{\mathbf{2}}=\hat{\mathbf{2}}^{\prime} \otimes \tilde{\mathbf{2}}^{\prime}=\mathbf{1} \oplus \mathbf{3}, \quad \hat{\mathbf{2}} \otimes \tilde{\mathbf{2}}^{\prime}=\hat{\mathbf{2}}^{\prime} \otimes \tilde{\mathbf{2}}=\mathbf{1}^{\prime} \oplus \mathbf{3}^{\prime}$ 
$2 \otimes 3=2 \otimes 3^{\prime}=2^{\prime} \otimes \hat{\mathbf{3}}=2^{\prime} \otimes \hat{\mathbf{3}}^{\prime}=\mathbf{3} \oplus \mathbf{3}^{\prime}, \quad 2 \otimes \hat{\mathbf{3}}=\mathbf{2} \otimes \hat{\mathbf{3}}^{\prime}=2^{\prime} \otimes \mathbf{3}=2^{\prime} \otimes \mathbf{3}^{\prime}=\hat{\mathbf{3}} \oplus \hat{\mathbf{3}}^{\prime}$,

$\hat{\mathbf{2}} \otimes \mathbf{3}=\hat{\mathbf{2}}^{\prime} \otimes \mathbf{3}^{\prime}=\tilde{\mathbf{2}} \otimes \hat{\mathbf{3}}^{\prime}=\tilde{\mathbf{2}}^{\prime} \otimes \hat{\mathbf{3}}=\hat{\mathbf{2}} \oplus \mathbf{4}, \quad \hat{\mathbf{2}} \otimes \mathbf{3}^{\prime}=\hat{\mathbf{2}}^{\prime} \otimes \mathbf{3}=\tilde{\mathbf{2}} \otimes \hat{\mathbf{3}}=\tilde{\mathbf{2}}^{\prime} \otimes \hat{\mathbf{3}}^{\prime}=\hat{\mathbf{2}}^{\prime} \oplus \mathbf{4}$,

$\hat{\mathbf{2}} \otimes \hat{\mathbf{3}}=\hat{\mathbf{2}}^{\prime} \otimes \hat{\mathbf{3}}^{\prime}=\tilde{\mathbf{2}} \otimes \mathbf{3}=\tilde{\mathbf{2}}^{\prime} \otimes \mathbf{3}^{\prime}=\tilde{\mathbf{2}} \oplus \mathbf{4}^{\prime}, \quad \hat{\mathbf{2}} \otimes \hat{\mathbf{3}}^{\prime}=\hat{\mathbf{2}}^{\prime} \otimes \hat{\mathbf{3}}=\tilde{\mathbf{2}} \otimes \mathbf{3}^{\prime}=\tilde{\mathbf{2}}^{\prime} \otimes \mathbf{3}=\tilde{\mathbf{2}}^{\prime} \oplus \mathbf{4}^{\prime}$,

$\mathbf{2} \otimes \mathbf{4}=\mathbf{2}^{\prime} \otimes \mathbf{4}^{\prime}=\hat{\mathbf{2}} \oplus \hat{\mathbf{2}}^{\prime} \oplus \mathbf{4}, \quad \mathbf{2} \otimes \mathbf{4}^{\prime}=\mathbf{2}^{\prime} \otimes \mathbf{4}=\tilde{\mathbf{2}} \oplus \tilde{\mathbf{2}}^{\prime} \oplus \mathbf{4}^{\prime}$,

$\hat{\mathbf{2}} \otimes \mathbf{4}=\hat{\mathbf{2}}^{\prime} \otimes \mathbf{4}=\tilde{\mathbf{2}} \otimes \mathbf{4}^{\prime}=\tilde{\mathbf{2}}^{\prime} \otimes \mathbf{4}^{\prime}=\mathbf{2}^{\prime} \oplus \hat{\mathbf{3}} \oplus \hat{\mathbf{3}}^{\prime}$,

$\hat{\mathbf{2}} \otimes \mathbf{4}^{\prime}=\hat{\mathbf{2}}^{\prime} \otimes \mathbf{4}^{\prime}=\tilde{\mathbf{2}} \otimes \mathbf{4}=\tilde{\mathbf{2}}^{\prime} \otimes \mathbf{4}=\mathbf{2} \oplus \mathbf{3} \oplus \mathbf{3}^{\prime}$,

$\mathbf{3} \otimes \mathbf{3}=\mathbf{3}^{\prime} \otimes \mathbf{3}^{\prime}=\hat{\mathbf{3}} \otimes \hat{\mathbf{3}}^{\prime}=\mathbf{1} \oplus \mathbf{2} \oplus \mathbf{3} \oplus \mathbf{3}^{\prime}, \quad 3 \otimes \mathbf{3}^{\prime}=\hat{\mathbf{3}} \otimes \hat{\mathbf{3}}=\hat{\mathbf{3}}^{\prime} \otimes \hat{\mathbf{3}}^{\prime}=\mathbf{1}^{\prime} \oplus \mathbf{2} \oplus \mathbf{3} \oplus \mathbf{3}^{\prime}$,

$\mathbf{3} \otimes \hat{\mathbf{3}}=\mathbf{3}^{\prime} \otimes \hat{\mathbf{3}}^{\prime}=\hat{\mathbf{1}} \oplus \mathbf{2}^{\prime} \oplus \hat{\mathbf{3}} \oplus \hat{\mathbf{3}}^{\prime}, \quad \mathbf{3} \otimes \hat{\mathbf{3}}^{\prime}=\mathbf{3}^{\prime} \otimes \hat{\mathbf{3}}=\hat{\mathbf{1}}^{\prime} \oplus \mathbf{2}^{\prime} \oplus \hat{\mathbf{3}} \oplus \hat{\mathbf{3}}^{\prime}$,

$\mathbf{3} \otimes \mathbf{4}=\mathbf{3}^{\prime} \otimes \mathbf{4}=\hat{\mathbf{3}} \otimes \mathbf{4}^{\prime}=\hat{\mathbf{3}}^{\prime} \otimes \mathbf{4}^{\prime}=\hat{\mathbf{2}} \oplus \hat{\mathbf{2}}^{\prime} \oplus \mathbf{4} \oplus \mathbf{4}$,

$3 \otimes 4^{\prime}=3^{\prime} \otimes 4^{\prime}=\hat{\mathbf{3}} \otimes \mathbf{4}=\hat{\mathbf{3}}^{\prime} \otimes \mathbf{4}=\tilde{\mathbf{2}} \oplus \tilde{\mathbf{2}}^{\prime} \oplus \mathbf{4}^{\prime} \oplus \mathbf{4}^{\prime}$,

$\mathbf{4} \otimes \mathbf{4}=\mathbf{4}^{\prime} \otimes \mathbf{4}^{\prime}=\hat{\mathbf{1}} \oplus \hat{\mathbf{1}}^{\prime} \oplus \mathbf{2}^{\prime} \oplus \hat{\mathbf{3}} \oplus \hat{\mathbf{3}} \oplus \hat{\mathbf{3}}^{\prime} \oplus \hat{\mathbf{3}}^{\prime}$,

$4 \otimes 4^{\prime}=1 \oplus \mathbf{1}^{\prime} \oplus \mathbf{2} \oplus \mathbf{3} \oplus \mathbf{3} \oplus \mathbf{3}^{\prime} \oplus \mathbf{3}^{\prime}$.

We list the Clebsch-Gordan coefficients of $\tilde{S}_{4}$ in following. All CG coefficients are expressed in the form of $\alpha \otimes \beta$, we use $\alpha_{i}\left(\beta_{i}\right)$ to denote the component of the left (right) basis vector $\alpha(\beta)$. The notations I, I I, I I I, and IV stand for singlet, doublet, triplet and quartet representations of $\tilde{S}_{4}$ respectively.

(i) $\mathbf{I} \otimes \mathbf{I} \rightarrow \mathbf{I}$,

$$
\left.\begin{array}{rrr}
\mathbf{1} \otimes \mathbf{1} \rightarrow \mathbf{1}_{\mathrm{s}}, & \mathbf{1} \otimes \mathbf{1}^{\prime} \rightarrow \mathbf{1}^{\prime} \\
\mathbf{1} \otimes \hat{\mathbf{1}} \rightarrow \hat{\mathbf{1}}, & \mathbf{1} \otimes \hat{\mathbf{1}}^{\prime} \rightarrow \hat{\mathbf{1}}^{\prime} \\
n=0 \quad \mathbf{1}^{\prime} \otimes \mathbf{1}^{\prime} \rightarrow \mathbf{1}_{\mathrm{s}}, & \mathbf{1}^{\prime} \otimes \hat{\mathbf{1}} \rightarrow \hat{\mathbf{1}}^{\prime} \\
\mathbf{1}^{\prime} \otimes \hat{\mathbf{1}}^{\prime} \rightarrow \hat{\mathbf{1}}, & \hat{\mathbf{1}} \otimes \hat{\mathbf{1}} \rightarrow \mathbf{1}_{\mathrm{s}}^{\prime} \\
\hat{\mathbf{1}} \otimes \hat{\mathbf{1}}^{\prime} \rightarrow \mathbf{1}, & \hat{\mathbf{1}}^{\prime} \otimes \hat{\mathbf{1}}^{\prime} \rightarrow \mathbf{1}_{\mathrm{s}}^{\prime}
\end{array}\right\} \quad \mathbf{I} \sim \alpha \beta
$$

(ii) $\mathbf{I} \otimes \mathbf{I I} \rightarrow \mathbf{I I}$,

$$
\left.\begin{array}{ll}
\mathbf{1} \otimes \mathbf{2} \rightarrow \mathbf{2}, & \mathbf{1} \otimes \mathbf{2}^{\prime} \rightarrow \mathbf{2}^{\prime} \\
\mathbf{1} \otimes \hat{\mathbf{2}} \rightarrow \hat{\mathbf{2}}, & \mathbf{1} \otimes \hat{\mathbf{2}}^{\prime} \rightarrow \hat{\mathbf{2}}^{\prime} \\
\mathbf{1} \otimes \tilde{\mathbf{2}} \rightarrow \tilde{\mathbf{2}}, & \mathbf{1} \otimes \tilde{\mathbf{2}}^{\prime} \rightarrow \tilde{\mathbf{2}}^{\prime} \\
& \mathbf{1}^{\prime} \otimes \hat{\mathbf{2}} \rightarrow \hat{\mathbf{2}}^{\prime}, \quad \mathbf{1}^{\prime} \otimes \hat{\mathbf{2}}^{\prime} \rightarrow \hat{\mathbf{2}} \\
n=0 & \mathbf{1}^{\prime} \otimes \tilde{\mathbf{2}} \rightarrow \tilde{\mathbf{2}}^{\prime}, \quad \mathbf{1}^{\prime} \otimes \tilde{\mathbf{2}}^{\prime} \rightarrow \tilde{\mathbf{2}} \\
\hat{\mathbf{1}} \otimes \mathbf{2} \rightarrow \mathbf{2}^{\prime}, \quad \hat{\mathbf{1}} \otimes \hat{\mathbf{2}} \rightarrow \tilde{\mathbf{2}} \\
\hat{\mathbf{1}} \otimes \hat{\mathbf{2}}^{\prime} \rightarrow \tilde{\mathbf{2}}^{\prime}, \hat{\mathbf{1}} \otimes \tilde{\mathbf{2}} \rightarrow \hat{\mathbf{2}}^{\prime} \\
\hat{\mathbf{1}} \otimes \tilde{\mathbf{2}}^{\prime} \rightarrow \hat{\mathbf{2}}, \quad \hat{\mathbf{1}}^{\prime} \otimes \mathbf{2}^{\prime} \rightarrow \mathbf{2} \\
\hat{\mathbf{1}}^{\prime} \otimes \hat{\mathbf{2}} \rightarrow \tilde{\mathbf{2}}^{\prime}, \hat{\mathbf{1}}^{\prime} \otimes \hat{\mathbf{2}}^{\prime} \rightarrow \tilde{\mathbf{2}} \\
\hat{\mathbf{1}}^{\prime} \otimes \tilde{\mathbf{2}} \rightarrow \hat{\mathbf{2}}, \quad \hat{\mathbf{1}}^{\prime} \otimes \tilde{\mathbf{2}}^{\prime} \rightarrow \hat{\mathbf{2}}^{\prime} \\
\mathbf{1}^{\prime} \otimes \mathbf{2} \rightarrow \mathbf{2}, \quad \mathbf{1}^{\prime} \otimes \mathbf{2}^{\prime} \rightarrow \mathbf{2}^{\prime} \\
\hat{\mathbf{1}} \otimes \mathbf{2}^{\prime} \rightarrow \mathbf{2}, \hat{\mathbf{1}}^{\prime} \otimes \mathbf{2} \rightarrow \mathbf{2}^{\prime}
\end{array}\right\}
$$

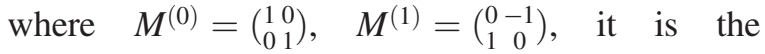
same below.

(iii) $\mathbf{I} \otimes \mathbf{I I ~ I ~} \rightarrow$ I I I,

$$
\left.\begin{array}{rr}
\mathbf{1} \otimes \mathbf{3} \rightarrow \mathbf{3}, & \mathbf{1} \otimes \mathbf{3}^{\prime} \rightarrow \mathbf{3}^{\prime} \\
\mathbf{1} \otimes \hat{\mathbf{3}} \rightarrow \hat{\mathbf{3}}, & \mathbf{1} \otimes \hat{\mathbf{3}}^{\prime} \rightarrow \hat{\mathbf{3}}^{\prime} \\
\mathbf{1}^{\prime} \otimes \mathbf{3} \rightarrow \mathbf{3}^{\prime}, & \mathbf{1}^{\prime} \otimes \mathbf{3}^{\prime} \rightarrow \mathbf{3} \\
\mathbf{1}^{\prime} \otimes \hat{\mathbf{3}} \rightarrow \hat{\mathbf{3}}^{\prime}, & \mathbf{1}^{\prime} \otimes \hat{\mathbf{3}}^{\prime} \rightarrow \hat{\mathbf{3}} \\
\hat{\mathbf{1}} \otimes \mathbf{3} \rightarrow \hat{\mathbf{3}}, & \hat{\mathbf{1}} \otimes \mathbf{3}^{\prime} \rightarrow \hat{\mathbf{3}}^{\prime} \\
\hat{\mathbf{1}} \otimes \hat{\mathbf{3}} \rightarrow \mathbf{3}^{\prime}, & \hat{\mathbf{1}} \otimes \hat{\mathbf{3}}^{\prime} \rightarrow \mathbf{3} \\
\hat{\mathbf{1}}^{\prime} \otimes \mathbf{3} \rightarrow \hat{\mathbf{3}}^{\prime}, & \hat{\mathbf{1}}^{\prime} \otimes \mathbf{3}^{\prime} \rightarrow \hat{\mathbf{3}} \\
\hat{\mathbf{1}}^{\prime} \otimes \hat{\mathbf{3}} \rightarrow \mathbf{3}, & \hat{\mathbf{1}}^{\prime} \otimes \hat{\mathbf{3}}^{\prime} \rightarrow \mathbf{3}^{\prime}
\end{array}\right\}
$$

(iv) $\mathbf{I} \otimes \mathbf{I V} \rightarrow \mathbf{I V}$

$$
\left.\begin{array}{cc} 
& \mathbf{1} \otimes 4 \rightarrow 4 \\
n=0 & \mathbf{1} \otimes 4^{\prime} \rightarrow 4^{\prime} \\
& \hat{\mathbf{1}} \otimes 4 \rightarrow 4^{\prime} \\
& \hat{\mathbf{1}}^{\prime} \otimes 4^{\prime} \rightarrow 4 \\
& \mathbf{1}^{\prime} \otimes 4 \rightarrow 4 \\
n=1 & \mathbf{1}^{\prime} \otimes 4^{\prime} \rightarrow 4^{\prime} \\
& \hat{\mathbf{1}} \otimes 4^{\prime} \rightarrow 4 \\
& \hat{\mathbf{1}}^{\prime} \otimes 4 \rightarrow 4^{\prime}
\end{array}\right\}
$$


(v) $\mathbf{I I} \otimes \mathbf{I I} \rightarrow \mathrm{I}_{1} \oplus \mathrm{I}_{2} \oplus \mathbf{I I}$,

$$
\left.\begin{array}{ll}
n=0 & \mathbf{2} \otimes \mathbf{2} \rightarrow \mathbf{1}_{\mathbf{a}}^{\prime} \oplus \mathbf{1}_{\mathbf{s}} \oplus \mathbf{2}_{\mathbf{s}} \\
& \mathbf{2} \otimes \mathbf{2}^{\prime} \rightarrow \hat{\mathbf{1}}^{\prime} \oplus \hat{\mathbf{1}} \oplus \mathbf{2}^{\prime} \\
n=1 & \mathbf{2}^{\prime} \otimes \mathbf{2}^{\prime} \rightarrow \mathbf{1}_{\mathbf{a}} \oplus \mathbf{1}_{\mathbf{s}}^{\prime} \oplus \mathbf{2}_{\mathbf{s}}
\end{array}\right\} \quad \begin{aligned}
& \mathbf{I}_{1} \sim \alpha_{1} \beta_{2}-\alpha_{2} \beta_{1} \\
& \mathbf{I}_{2} \sim \alpha_{1} \beta_{1}+\alpha_{2} \beta_{2} \\
& \mathbf{I} \mathbf{I} \sim M^{(n)}\left(\begin{array}{c}
-\alpha_{1} \beta_{1}+\alpha_{2} \beta_{2} \\
\alpha_{1} \beta_{2}+\alpha_{2} \beta_{1}
\end{array}\right)
\end{aligned}
$$

(vi) $\mathbf{I I} \otimes \mathbf{I I} \rightarrow \mathbf{I V}$,

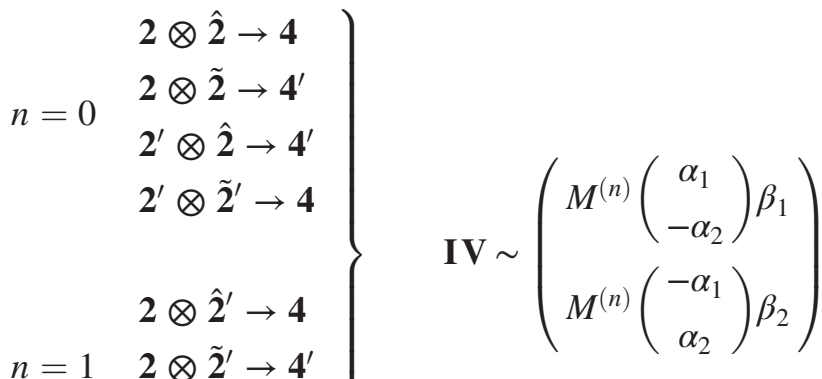

$$
\begin{aligned}
& \mathbf{2}^{\prime} \otimes \hat{\mathbf{2}}^{\prime} \rightarrow \mathbf{4}^{\prime} \\
& \mathbf{2}^{\prime} \otimes \tilde{\mathbf{2}} \rightarrow 4
\end{aligned}
$$

(vii) I I $\otimes \mathbf{I I} \rightarrow \mathbf{I} \oplus \mathbf{I} \mathbf{I}$,

$$
\begin{aligned}
& \hat{\mathbf{2}} \otimes \hat{\mathbf{2}} \rightarrow \hat{\mathbf{1}}_{\mathbf{a}}^{\prime} \oplus \hat{\mathbf{3}}_{\mathbf{s}}^{\prime} \\
& \hat{\mathbf{2}} \otimes \hat{\mathbf{2}}^{\prime} \rightarrow \hat{\mathbf{1}} \oplus \hat{\mathbf{3}} \\
& \hat{\mathbf{2}} \otimes \tilde{\mathbf{2}} \rightarrow \mathbf{1} \oplus \mathbf{3} \\
& \hat{\mathbf{2}} \otimes \tilde{\mathbf{2}}^{\prime} \rightarrow \mathbf{1}^{\prime} \oplus \mathbf{3}^{\prime} \\
& n=0 \quad \begin{array}{l}
\hat{\mathbf{2}}^{\prime} \otimes \hat{\mathbf{2}}^{\prime} \rightarrow \hat{\mathbf{1}}_{\mathbf{a}}^{\prime} \oplus \hat{\mathbf{3}}_{\mathbf{s}}^{\prime} \\
\hat{\mathbf{2}}^{\prime} \otimes \tilde{\mathbf{2}} \rightarrow \mathbf{1}^{\prime} \oplus \mathbf{3}^{\prime}
\end{array} \\
& \hat{\mathbf{2}}^{\prime} \otimes \tilde{\mathbf{2}}^{\prime} \rightarrow \mathbf{1} \oplus \mathbf{3} \\
& \tilde{\mathbf{2}} \otimes \tilde{\mathbf{2}} \rightarrow \hat{\mathbf{1}}_{\mathrm{a}} \oplus \hat{\mathbf{3}}_{\mathrm{s}} \\
& \tilde{\mathbf{2}} \otimes \tilde{\mathbf{2}}^{\prime} \rightarrow \hat{\mathbf{1}}^{\prime} \oplus \hat{\mathbf{3}}^{\prime} \\
& \tilde{\mathbf{2}}^{\prime} \otimes \tilde{\mathbf{2}}^{\prime} \rightarrow \hat{\mathbf{1}}_{\mathrm{a}} \oplus \hat{\mathbf{3}}_{\mathrm{s}} \\
& \mathbf{I} \sim \alpha_{1} \beta_{2}-\alpha_{2} \beta_{1} \\
& \text { II I } \sim\left(\begin{array}{c}
\alpha_{1} \beta_{2}+\alpha_{2} \beta_{1} \\
-\sqrt{2} \alpha_{2} \beta_{2} \\
\sqrt{2} \alpha_{1} \beta_{1}
\end{array}\right)
\end{aligned}
$$

(viii) I I $\otimes \mathbf{I ~ I ~ I ~} \rightarrow$ I I I $_{1} \oplus \mathbf{I I ~ I ~}_{2}$,

$$
\left.\begin{array}{rl}
\mathbf{2} & \otimes \mathbf{3} \rightarrow \mathbf{3} \oplus \mathbf{3}^{\prime} \\
\mathbf{2} \otimes \mathbf{3}^{\prime} \rightarrow \mathbf{3}^{\prime} \oplus \mathbf{3} \\
\mathbf{2} \otimes \hat{\mathbf{3}} \rightarrow \hat{\mathbf{3}} \oplus \hat{\mathbf{3}}^{\prime} \\
\mathbf{2} \otimes \hat{\mathbf{3}}^{\prime} \rightarrow \hat{\mathbf{3}}^{\prime} \oplus \hat{\mathbf{3}} \\
\mathbf{2}^{\prime} \otimes \mathbf{3} \rightarrow \hat{\mathbf{3}} \oplus \hat{\mathbf{3}}^{\prime} \\
\mathbf{2}^{\prime} \otimes \mathbf{3}^{\prime} \rightarrow \hat{\mathbf{3}}^{\prime} \oplus \hat{\mathbf{3}} \\
\mathbf{2}^{\prime} \otimes \hat{\mathbf{3}} \rightarrow \mathbf{3}^{\prime} \oplus \mathbf{3} \\
\mathbf{2}^{\prime} \otimes \hat{\mathbf{3}}^{\prime} \rightarrow \mathbf{3} \oplus \mathbf{3}^{\prime}
\end{array}\right\} \quad \quad \mathbf{I I I}_{\mathbf{1}} \sim\left(\begin{array}{c}
2 \alpha_{1} \beta_{1} \\
-\alpha_{1} \beta_{2}+\sqrt{3} \alpha_{2} \beta_{3} \\
-\alpha_{1} \beta_{3}+\sqrt{3} \alpha_{2} \beta_{2}
\end{array}\right)
$$


(ix) $\mathbf{I I} \otimes \mathbf{I} \mathbf{I} \mathbf{I} \rightarrow \mathbf{I I} \oplus \mathbf{I} \mathbf{V}$,

$$
\left.\begin{array}{l}
\hat{\mathbf{2}} \otimes \mathbf{3}^{\prime} \rightarrow \hat{\mathbf{2}}^{\prime} \oplus \mathbf{4} \\
\hat{\mathbf{2}} \otimes \hat{\mathbf{3}}^{\prime} \rightarrow \tilde{\mathbf{2}}^{\prime} \oplus \mathbf{4}^{\prime} \\
\hat{\mathbf{2}}^{\prime} \otimes \mathbf{3} \rightarrow \hat{\mathbf{2}}^{\prime} \oplus \mathbf{4} \\
\hat{\mathbf{2}}^{\prime} \otimes \hat{\mathbf{3}} \rightarrow \tilde{\mathbf{2}}^{\prime} \oplus \mathbf{4}^{\prime} \\
\tilde{\mathbf{2}} \otimes \mathbf{3}^{\prime} \rightarrow \tilde{\mathbf{2}}^{\prime} \oplus \mathbf{4}^{\prime} \\
\tilde{\mathbf{2}} \otimes \hat{\mathbf{3}} \rightarrow \hat{\mathbf{2}}^{\prime} \oplus \mathbf{4} \\
\tilde{\mathbf{2}}^{\prime} \otimes \mathbf{3} \rightarrow \tilde{\mathbf{2}}^{\prime} \oplus \mathbf{4}^{\prime} \\
\tilde{\mathbf{2}}^{\prime} \otimes \hat{\mathbf{3}}^{\prime} \rightarrow \hat{\mathbf{2}}^{\prime} \oplus \mathbf{4} \\
\hat{\mathbf{2}} \otimes \mathbf{3} \rightarrow \hat{\mathbf{2}} \oplus \mathbf{4} \\
\hat{\mathbf{2}} \otimes \hat{\mathbf{3}} \rightarrow \tilde{\mathbf{2}} \oplus \mathbf{4}^{\prime} \\
\hat{\mathbf{2}}^{\prime} \otimes \mathbf{3}^{\prime} \rightarrow \hat{\mathbf{2}} \oplus \mathbf{4} \\
\hat{\mathbf{2}}^{\prime} \otimes \hat{\mathbf{3}}^{\prime} \rightarrow \tilde{\mathbf{2}} \oplus \mathbf{4}^{\prime} \\
\tilde{\mathbf{2}} \otimes \mathbf{3} \rightarrow \tilde{\mathbf{2}} \oplus \mathbf{4}^{\prime} \\
\tilde{\mathbf{2}} \otimes \hat{\mathbf{3}}^{\prime} \rightarrow \hat{\mathbf{2}} \oplus \mathbf{4} \\
\tilde{\mathbf{2}}^{\prime} \otimes \mathbf{3}^{\prime} \rightarrow \tilde{\mathbf{2}} \oplus \mathbf{4}^{\prime} \\
\tilde{\mathbf{2}}^{\prime} \otimes \hat{\mathbf{3}} \rightarrow \hat{\mathbf{2}} \oplus \mathbf{4}
\end{array}\right\} \quad \mathbf{I I} \sim\left(\begin{array}{c}
-\alpha_{1} \beta_{1}+\sqrt{2} \alpha_{2} \beta_{3} \\
\sqrt{2} \alpha_{1} \beta_{2}+\alpha_{2} \beta_{1}
\end{array}\right)
$$

$(\mathrm{x}) \quad \mathrm{II} \otimes \mathbf{I V} \rightarrow \mathrm{II}_{\mathbf{1}} \oplus \mathbf{I} \mathbf{I}_{\mathbf{2}} \oplus \mathbf{I V}$,

$$
\left.\begin{array}{ll}
n=0 & \mathbf{2}^{\prime} \otimes \mathbf{4}^{\prime} \rightarrow \hat{\mathbf{2}} \oplus \hat{\mathbf{2}}^{\prime} \oplus \mathbf{4} \\
& \mathbf{2} \otimes \mathbf{4} \rightarrow \hat{\mathbf{2}}^{\prime} \oplus \hat{\mathbf{2}} \oplus \mathbf{4} \\
n=1 & \mathbf{2} \otimes \mathbf{4}^{\prime} \rightarrow \tilde{\mathbf{2}}^{\prime} \oplus \tilde{\mathbf{2}} \oplus \mathbf{4}^{\prime} \\
& \mathbf{2}^{\prime} \otimes \mathbf{4} \rightarrow \tilde{\mathbf{2}}^{\prime} \oplus \tilde{\mathbf{2}} \oplus \mathbf{4}^{\prime}
\end{array}\right\}
$$

$$
\begin{aligned}
& \mathbf{I I}_{\mathbf{1}} \sim\left(\begin{array}{c}
\alpha_{1} \beta_{2}+\alpha_{2} \beta_{1} \\
-\alpha_{1} \beta_{4}-\alpha_{2} \beta_{3}
\end{array}\right) \\
& \mathbf{I I}_{\mathbf{2}} \sim\left(\begin{array}{c}
\alpha_{1} \beta_{1}-\alpha_{2} \beta_{2} \\
-\alpha_{1} \beta_{3}+\alpha_{2} \beta_{4}
\end{array}\right) \\
& \mathbf{I V} \sim\left(\begin{array}{c}
\boldsymbol{M}^{(n)}\left(\begin{array}{c}
\alpha_{1} \beta_{2}-\alpha_{2} \beta_{1} \\
\alpha_{1} \beta_{1}+\alpha_{2} \beta_{2}
\end{array}\right) \\
\boldsymbol{M}^{(n)}\left(\begin{array}{c}
\alpha_{1} \beta_{4}-\alpha_{2} \beta_{3} \\
\alpha_{1} \beta_{3}+\alpha_{2} \beta_{4}
\end{array}\right)
\end{array}\right)
\end{aligned}
$$

(xi) I I $\otimes \mathbf{I V} \rightarrow \mathbf{I I} \oplus \mathbf{I I I}_{\mathbf{1}} \oplus \mathbf{I I I}_{\mathbf{2}}$,

$$
\begin{aligned}
& \left.\begin{array}{l}
\hat{\mathbf{2}} \otimes \mathbf{4} \rightarrow \mathbf{2}^{\prime} \oplus \hat{\mathbf{3}}^{\prime} \oplus \hat{\mathbf{3}} \\
\hat{\mathbf{2}}^{\prime} \otimes \mathbf{4}^{\prime} \rightarrow \mathbf{2} \oplus \mathbf{3}^{\prime} \oplus \mathbf{3}
\end{array}\right) \quad \mathbf{I} \mathbf{I} \sim M^{(n)}\left(\begin{array}{c}
\alpha_{1} \beta_{4}+\alpha_{2} \beta_{2} \\
\alpha_{1} \beta_{3}+\alpha_{2} \beta_{1}
\end{array}\right) \\
& n=0 \quad \begin{array}{l}
\mathbf{2}^{\prime} \otimes 4^{\prime} \rightarrow \mathbf{2} \oplus \mathbf{3}^{\prime} \oplus \mathbf{3} \\
\tilde{\mathbf{2}}^{\prime} \otimes \mathbf{4} \rightarrow \mathbf{2} \oplus \mathbf{3}^{\prime} \oplus \mathbf{3}
\end{array} \\
& \tilde{\mathbf{2}}^{\prime} \otimes \mathbf{4}^{\prime} \rightarrow \mathbf{2}^{\prime} \oplus \hat{\mathbf{3}}^{\prime} \oplus \hat{\mathbf{3}} \\
& \hat{\mathbf{2}} \otimes 4^{\prime} \rightarrow \mathbf{2} \oplus \mathbf{3} \oplus \mathbf{3}^{\prime} \\
& n=1 \quad \begin{array}{l}
\hat{\mathbf{2}}^{\prime} \otimes \mathbf{4} \rightarrow \mathbf{2}^{\prime} \oplus \hat{\mathbf{3}} \oplus \hat{\mathbf{3}}^{\prime} \\
\tilde{\mathbf{2}} \otimes \mathbf{4} \rightarrow \mathbf{2} \oplus \mathbf{3} \oplus \mathbf{3}^{\prime} \\
\tilde{\mathbf{2}} \otimes \mathbf{4}^{\prime} \rightarrow \mathbf{2}^{\prime} \oplus \hat{\mathbf{3}} \oplus \hat{\mathbf{3}}^{\prime}
\end{array} \\
& \begin{array}{c}
\mathbf{I I I}_{\mathbf{1}} \sim\left(\begin{array}{c}
\sqrt{2} \alpha_{1} \beta_{3}-\sqrt{2} \alpha_{2} \beta_{1} \\
\sqrt{3} \alpha_{1} \beta_{2}+\alpha_{2} \beta_{3} \\
\alpha_{1} \beta_{1}+\sqrt{3} \alpha_{2} \beta_{4}
\end{array}\right) \\
\mathbf{I I I}_{\mathbf{2}} \sim\left(\begin{array}{c}
\sqrt{2} \alpha_{1} \beta_{4}-\sqrt{2} \alpha_{2} \beta_{2} \\
-\sqrt{3} \alpha_{1} \beta_{1}+\alpha_{2} \beta_{4} \\
\alpha_{1} \beta_{2}-\sqrt{3} \alpha_{2} \beta_{3}
\end{array}\right)
\end{array}
\end{aligned}
$$


(xii) I I I $\otimes \mathbf{I ~ I ~ I ~} \rightarrow \mathbf{I} \oplus \mathbf{I} \mathbf{I} \oplus \mathbf{I} \mathbf{I} \mathbf{I}_{1} \oplus \mathbf{I} \mathbf{I} \mathbf{I}_{2}$,

$$
\begin{aligned}
& 3 \otimes 3 \rightarrow 1_{\mathrm{s}} \oplus 2_{\mathrm{s}} \oplus 3_{\mathrm{a}} \oplus 3_{\mathrm{s}}^{\prime} \\
& \mathbf{3} \otimes \hat{\mathbf{3}} \rightarrow \hat{\mathbf{1}} \oplus \mathbf{2}^{\prime} \oplus \hat{\mathbf{3}} \oplus \hat{\mathbf{3}}^{\prime} \\
& n=0 \quad \mathbf{3}^{\prime} \otimes \mathbf{3}^{\prime} \rightarrow \mathbf{1}_{\mathrm{s}} \oplus \mathbf{2}_{\mathrm{s}} \oplus \mathbf{3}_{\mathrm{a}} \oplus \mathbf{3}_{\mathrm{s}}^{\prime} \\
& \mathbf{3}^{\prime} \otimes \hat{\mathbf{3}}^{\prime} \rightarrow \hat{\mathbf{1}} \oplus \mathbf{2}^{\prime} \oplus \hat{\mathbf{3}} \oplus \hat{\mathbf{3}}^{\prime} \\
& \hat{\mathbf{3}} \otimes \hat{\mathbf{3}}^{\prime} \rightarrow \mathbf{1} \oplus \mathbf{2} \oplus \mathbf{3} \oplus \mathbf{3}^{\prime} \\
& 3 \otimes 3^{\prime} \rightarrow 1^{\prime} \oplus 2 \oplus 3^{\prime} \oplus 3 \\
& \mathbf{3} \otimes \hat{\mathbf{3}}^{\prime} \rightarrow \hat{\mathbf{1}}^{\prime} \oplus \mathbf{2}^{\prime} \oplus \hat{\mathbf{3}}^{\prime} \oplus \hat{\mathbf{3}} \\
& n=1 \quad \mathbf{3}^{\prime} \otimes \hat{\mathbf{3}} \rightarrow \hat{\mathbf{1}}^{\prime} \oplus \mathbf{2}^{\prime} \oplus \hat{\mathbf{3}}^{\prime} \oplus \hat{\mathbf{3}} \\
& \hat{\mathbf{3}} \otimes \hat{\mathbf{3}} \rightarrow \mathbf{1}_{\mathrm{s}}^{\prime} \oplus \mathbf{2}_{\mathrm{s}} \oplus \mathbf{3}_{\mathrm{a}}^{\prime} \oplus \mathbf{3}_{\mathrm{s}} \\
& \hat{\mathbf{3}}^{\prime} \otimes \hat{\mathbf{3}}^{\prime} \rightarrow \mathbf{1}_{\mathrm{s}}^{\prime} \oplus \mathbf{2}_{\mathrm{s}} \oplus \mathbf{3}_{\mathrm{a}}^{\prime} \oplus \mathbf{3}_{\mathrm{s}} \\
& \mathbf{I} \sim \alpha_{1} \beta_{1}+\alpha_{2} \beta_{3}+\alpha_{3} \beta_{2} \\
& \mathbf{I} \mathbf{I} \sim M^{(n)}\left(\begin{array}{c}
2 \alpha_{1} \beta_{1}-\alpha_{2} \beta_{3}-\alpha_{3} \beta_{2} \\
\sqrt{3} \alpha_{2} \beta_{2}+\sqrt{3} \alpha_{3} \beta_{3}
\end{array}\right) \\
& \mathbf{I I I}_{\mathbf{1}} \sim\left(\begin{array}{c}
\alpha_{2} \beta_{3}-\alpha_{3} \beta_{2} \\
\alpha_{1} \beta_{2}-\alpha_{2} \beta_{1} \\
-\alpha_{1} \beta_{3}+\alpha_{3} \beta_{1}
\end{array}\right) \\
& \mathbf{I I I}_{2} \sim\left(\begin{array}{c}
\alpha_{2} \beta_{2}-\alpha_{3} \beta_{3} \\
-\alpha_{1} \beta_{3}-\alpha_{3} \beta_{1} \\
\alpha_{1} \beta_{2}+\alpha_{2} \beta_{1}
\end{array}\right)
\end{aligned}
$$

(xiii) I I I $\otimes I V \rightarrow I I_{1} \oplus I_{2} \oplus \mathbf{I} V_{1} \oplus \mathbf{I} \mathbf{V}_{2}$

$$
\begin{aligned}
& \mathbf{I I}_{\mathbf{1}} \sim\left(\begin{array}{c}
\sqrt{2} \alpha_{1} \beta_{1}-\sqrt{3} \alpha_{2} \beta_{4}-\alpha_{3} \beta_{3} \\
\sqrt{2} \alpha_{1} \beta_{3}+\alpha_{2} \beta_{1}+\sqrt{3} \alpha_{3} \beta_{2}
\end{array}\right) \\
& \mathbf{3}^{\prime} \otimes \mathbf{4} \rightarrow \hat{\mathbf{2}}^{\prime} \oplus \hat{\mathbf{2}} \oplus \mathbf{4}_{\mathrm{I}} \oplus \mathbf{4}_{\mathrm{II}} \\
& n=0 \quad \mathbf{3}^{\prime} \otimes \mathbf{4}^{\prime} \rightarrow \tilde{\mathbf{2}}^{\prime} \oplus \tilde{\mathbf{2}} \oplus \mathbf{4}_{\mathrm{I}}^{\prime} \oplus \mathbf{4}_{\mathrm{II}}^{\prime} \\
& \hat{\mathbf{3}} \otimes \mathbf{4}^{\prime} \rightarrow \hat{\mathbf{2}}^{\prime} \oplus \hat{\mathbf{2}} \oplus \mathbf{4}_{\mathrm{I}} \oplus \mathbf{4}_{\mathrm{II}} \\
& \hat{\mathbf{3}}^{\prime} \otimes \mathbf{4} \rightarrow \tilde{\mathbf{2}}^{\prime} \oplus \tilde{\mathbf{2}} \oplus \mathbf{4}_{\mathrm{I}}^{\prime} \oplus \mathbf{4}_{\mathrm{II}}^{\prime} \\
& \mathbf{3} \otimes \mathbf{4} \rightarrow \hat{\mathbf{2}} \oplus \hat{\mathbf{2}}^{\prime} \oplus \mathbf{4}_{\mathrm{I}} \oplus \mathbf{4}_{\text {II }} \\
& n=1 \quad \mathbf{3} \otimes \mathbf{4}^{\prime} \rightarrow \tilde{\mathbf{2}} \oplus \tilde{\mathbf{2}}^{\prime} \oplus \mathbf{4}_{\mathrm{I}}^{\prime} \oplus \mathbf{4}_{\text {II }}^{\prime} \\
& \hat{\mathbf{3}} \otimes \mathbf{4} \rightarrow \tilde{\mathbf{2}} \oplus \tilde{\mathbf{2}}^{\prime} \oplus \mathbf{4}_{\mathrm{I}}^{\prime} \oplus \mathbf{4}_{\mathrm{II}}^{\prime} \\
& \hat{\mathbf{3}}^{\prime} \otimes \mathbf{4}^{\prime} \rightarrow \hat{\mathbf{2}} \oplus \hat{\mathbf{2}}^{\prime} \oplus \mathbf{4}_{\mathrm{I}} \oplus \mathbf{4}_{\mathrm{II}} \\
& \mathbf{I I}_{2} \sim\left(\begin{array}{c}
\sqrt{2} \alpha_{1} \beta_{2}+\sqrt{3} \alpha_{2} \beta_{3}-\alpha_{3} \beta_{4} \\
\sqrt{2} \alpha_{1} \beta_{4}+\alpha_{2} \beta_{2}-\sqrt{3} \alpha_{3} \beta_{1}
\end{array}\right) \\
& \mathbf{I} \mathbf{V}_{\mathbf{1}} \sim\left(\begin{array}{c}
M^{(n)}\left(\begin{array}{c}
\alpha_{1} \beta_{2}-\sqrt{6} \alpha_{2} \beta_{3}-2 \sqrt{2} \alpha_{3} \beta_{4} \\
3 \alpha_{1} \beta_{1}+\sqrt{6} \alpha_{2} \beta_{4}
\end{array}\right) \\
M^{(n)}\left(\begin{array}{c}
-\alpha_{1} \beta_{4}-2 \sqrt{2} \alpha_{2} \beta_{2}-\sqrt{6} \alpha_{3} \beta_{1} \\
-3 \alpha_{1} \beta_{3}+\sqrt{6} \alpha_{3} \beta_{2}
\end{array}\right)
\end{array}\right) \\
& \mathbf{I} \mathbf{V}_{2} \sim\left(\begin{array}{c}
M^{(n)}\left(\begin{array}{c}
3 \alpha_{1} \beta_{2}-\sqrt{6} \alpha_{2} \beta_{3} \\
\alpha_{1} \beta_{1}+\sqrt{6} \alpha_{2} \beta_{4}-2 \sqrt{2} \alpha_{3} \beta_{3}
\end{array}\right) \\
M^{(n)}\left(\begin{array}{c}
-3 \alpha_{1} \beta_{4}-\sqrt{6} \alpha_{3} \beta_{1} \\
-\alpha_{1} \beta_{3}-2 \sqrt{2} \alpha_{2} \beta_{1}+\sqrt{6} \alpha_{3} \beta_{2}
\end{array}\right)
\end{array}\right)
\end{aligned}
$$


(xiv) $\mathbf{I V} \otimes \mathbf{I V} \rightarrow \mathbf{I}_{1} \oplus \mathbf{I}_{2} \oplus \mathbf{I I} \oplus \mathbf{I} \mathbf{I} \mathbf{I}_{1} \oplus \mathbf{I} \mathbf{I} \mathbf{I}_{2} \oplus \mathbf{I} \mathbf{I}_{\mathbf{3}} \oplus \mathbf{I} \mathbf{I I}_{\mathbf{4}}$,

$$
\left.\begin{array}{ll}
n=0 & \mathbf{4} \otimes \mathbf{4} \rightarrow \hat{\mathbf{1}}_{\mathrm{s}} \oplus \hat{\mathbf{1}}_{\mathrm{a}}^{\prime} \oplus \mathbf{2}_{\mathrm{a}}^{\prime} \oplus \hat{\mathbf{3}}_{\mathrm{a}} \oplus \hat{\mathbf{3}}_{\mathrm{s}} \oplus \hat{\mathbf{3}}_{\mathrm{sI}}^{\prime} \oplus \hat{\mathbf{3}}_{\mathrm{sII}}^{\prime} \\
n=1 & \mathbf{4} \otimes \mathbf{4}^{\prime} \rightarrow \mathbf{1}^{\prime} \oplus \mathbf{1} \oplus \mathbf{2} \oplus \mathbf{3}_{\mathrm{II}}^{\prime} \oplus \mathbf{3}_{\mathrm{I}}^{\prime} \oplus \mathbf{3}_{\mathrm{I}} \oplus \mathbf{3}_{\mathrm{II}} \\
& \mathbf{4}^{\prime} \otimes \mathbf{4}^{\prime} \rightarrow \hat{\mathbf{1}}_{\mathrm{s}}^{\prime} \oplus \hat{\mathbf{1}}_{\mathrm{a}} \oplus \mathbf{2}_{\mathrm{a}}^{\prime} \oplus \hat{\mathbf{3}}_{\mathrm{a}}^{\prime} \oplus \hat{\mathbf{3}}_{\mathrm{s}}^{\prime} \oplus \hat{\mathbf{3}}_{\mathrm{SI}} \oplus \hat{\mathbf{3}}_{\mathrm{SII}}
\end{array}\right\}
$$

$$
\begin{aligned}
& \mathbf{I}_{1} \sim \alpha_{1} \beta_{4}-\alpha_{2} \beta_{3}-\alpha_{3} \beta_{2}+\alpha_{4} \beta_{1} \\
& \mathbf{I}_{2} \sim \alpha_{1} \beta_{3}+\alpha_{2} \beta_{4}-\alpha_{3} \beta_{1}-\alpha_{4} \beta_{2} \\
& \mathbf{I} \mathbf{I} \sim M^{(n)}\left(\begin{array}{c}
-\alpha_{1} \beta_{4}-\alpha_{2} \beta_{3}+\alpha_{3} \beta_{2}+\alpha_{4} \beta_{1} \\
\alpha_{1} \beta_{3}-\alpha_{2} \beta_{4}-\alpha_{3} \beta_{1}+\alpha_{4} \beta_{2}
\end{array}\right) \\
& \mathbf{I I I}_{\mathbf{1}} \sim\left(\begin{array}{c}
\alpha_{1} \beta_{4}-\alpha_{2} \beta_{3}+\alpha_{3} \beta_{2}-\alpha_{4} \beta_{1} \\
\sqrt{2} \alpha_{3} \beta_{4}-\sqrt{2} \alpha_{4} \beta_{3} \\
-\sqrt{2} \alpha_{1} \beta_{2}+\sqrt{2} \alpha_{2} \beta_{1}
\end{array}\right) \\
& \mathbf{I I I}_{2} \sim\left(\begin{array}{c}
\sqrt{2} \alpha_{1} \beta_{4}+\sqrt{2} \alpha_{2} \beta_{3}+\sqrt{2} \alpha_{3} \beta_{2}+\sqrt{2} \alpha_{4} \beta_{1} \\
\sqrt{3} \alpha_{1} \beta_{1}-\sqrt{3} \alpha_{2} \beta_{2}-\alpha_{3} \beta_{4}-\alpha_{4} \beta_{3} \\
\alpha_{1} \beta_{2}+\alpha_{2} \beta_{1}-\sqrt{3} \alpha_{3} \beta_{3}+\sqrt{3} \alpha_{4} \beta_{4}
\end{array}\right) \\
& \mathbf{I I I}_{\mathbf{3}} \sim\left(\begin{array}{c}
\alpha_{1} \beta_{3}-3 \alpha_{2} \beta_{4}+\alpha_{3} \beta_{1}-3 \alpha_{4} \beta_{2} \\
-\sqrt{6} \alpha_{1} \beta_{2}-\sqrt{6} \alpha_{2} \beta_{1}-2 \sqrt{2} \alpha_{3} \beta_{3} \\
2 \sqrt{2} \alpha_{1} \beta_{1}+\sqrt{6} \alpha_{3} \beta_{4}+\sqrt{6} \alpha_{4} \beta_{3}
\end{array}\right) \\
& \mathbf{I I I}_{\mathbf{4}} \sim\left(\begin{array}{c}
\alpha_{1} \beta_{3}+\alpha_{2} \beta_{4}+\alpha_{3} \beta_{1}+\alpha_{4} \beta_{2} \\
\sqrt{2} \alpha_{3} \beta_{3}+\sqrt{2} \alpha_{4} \beta_{4} \\
-\sqrt{2} \alpha_{1} \beta_{1}-\sqrt{2} \alpha_{2} \beta_{2}
\end{array}\right)
\end{aligned}
$$

[1] F. Feruglio, Are neutrino masses modular forms?, in From My Vast Repertoire ...: Guido Altarelli's Legacy, edited by A. Levy, S. Forte, and G. Ridolfi (World Scientic Publishing Co Pte Ltd., Singapore, 2019), pp. 227-266.

[2] S. Kikuchi, T. Kobayashi, S. Takada, T. H. Tatsuishi, and H. Uchida, Revisiting modular symmetry in magnetized torus and orbifold compactifications, Phys. Rev. D 102, 105010 (2020).

[3] S. Kikuchi, T. Kobayashi, H. Otsuka, S. Takada, and H. Uchida, Modular symmetry by orbifolding magnetized $T^{2} \times T^{2}$ : Realization of double cover of $\Gamma_{N}$, J. High Energy Phys. 11 (2020) 101.

[4] M.-C. Chen, S. Ramos-Snchez, and M. Ratz, A note on the predictions of models with modular flavor symmetries, Phys. Lett. B 801, 135153 (2020).

[5] H. P. Nilles, S. Ramos-Snchez, and P. K. Vaudrevange, Eclectic flavor groups, J. High Energy Phys. 02 (2020) 045.

[6] H. P. Nilles, S. Ramos-Sanchez, and P. K. Vaudrevange, Lessons from eclectic flavor symmetries, Nucl. Phys. B957, 115098 (2020).

[7] H. P. Nilles, S. Ramos-Snchez, and P. K. Vaudrevange, Eclectic flavor scheme from ten-dimensional string theory-I. Basic results, Phys. Lett. B 808, 135615 (2020).

[8] T. Kobayashi, K. Tanaka, and T. H. Tatsuishi, Neutrino mixing from finite modular groups, Phys. Rev. D 98, 016004 (2018).
[9] T. Kobayashi, Y. Shimizu, K. Takagi, M. Tanimoto, T. H. Tatsuishi, and H. Uchida, Finite modular subgroups for fermion mass matrices and baryon/lepton number violation, Phys. Lett. B 794, 114 (2019).

[10] T. Kobayashi, Y. Shimizu, K. Takagi, M. Tanimoto, and T. H. Tatsuishi, Modular $S_{3}$ invariant flavor model in SU(5) GUT, Prog. Theor. Exp. Phys. (2020) 053B05.

[11] H. Okada and Y. Orikasa, Modular $S_{3}$ symmetric radiative seesaw model, Phys. Rev. D 100, 115037 (2019).

[12] J. C. Criado and F. Feruglio, Modular invariance faces precision neutrino data, SciPost Phys. 5, 042 (2018).

[13] T. Kobayashi, N. Omoto, Y. Shimizu, K. Takagi, M. Tanimoto, and T.H. Tatsuishi, Modular $\mathrm{A}_{4}$ invariance and neutrino mixing, J. High Energy Phys. 11 (2018) 196.

[14] F. J. de Anda, S. F. King, and E. Perdomo, $S U(5)$ grand unified theory with $A_{4}$ modular symmetry, Phys. Rev. D 101, 015028 (2020).

[15] H. Okada and M. Tanimoto, $C P$ violation of quarks in $A_{4}$ modular invariance, Phys. Lett. B 791, 54 (2019).

[16] P. Novichkov, S. Petcov, and M. Tanimoto, Trimaximal neutrino mixing from modular a4 invariance with residual symmetries, Phys. Lett. B 793, 247 (2019).

[17] T. Nomura and H. Okada, A modular $A_{4}$ symmetric model of dark matter and neutrino, Phys. Lett. B 797, 134799 (2019). 
[18] H. Okada and M. Tanimoto, Towards unification of quark and lepton flavors in $A_{4}$ modular invariance, arXiv: 1905.13421.

[19] T. Nomura and H. Okada, A two loop induced neutrino mass model with modular $A_{4}$ symmetry, arXiv:1906.03927.

[20] G.-J. Ding, S. F. King, and X.-G. Liu, Modular $\mathrm{A}_{4}$ symmetry models of neutrinos and charged leptons, J. High Energy Phys. 09 (2019) 074.

[21] H. Okada and Y. Orikasa, A radiative seesaw model in modular $A_{4}$ symmetry, arXiv:1907.13520.

[22] T. Nomura, H. Okada, and O. Popov, A modular $A_{4}$ symmetric scotogenic model, Phys. Lett. B 803, 135294 (2020).

[23] T. Kobayashi, Y. Shimizu, K. Takagi, M. Tanimoto, and T. H. Tatsuishi, $A_{4}$ lepton flavor model and modulus stabilization from $S_{4}$ modular symmetry, Phys. Rev. D 100, 115045 (2019); Erratum, Phys. Rev. D 101, 039904 (2020)].

[24] T. Asaka, Y. Heo, T. H. Tatsuishi, and T. Yoshida, Modular $A_{4}$ invariance and leptogenesis, J. High Energy Phys. 01 (2020) 144.

[25] G.-J. Ding, S. F. King, X.-G. Liu, and J.-N. Lu, Modular $\mathrm{S}_{4}$ and $\mathrm{A}_{4}$ symmetries and their fixed points: New predictive examples of lepton mixing, J. High Energy Phys. 12 (2019) 030.

[26] D. Zhang, A modular $A_{4}$ symmetry realization of two-zero textures of the Majorana neutrino mass matrix, Nucl. Phys. B952, 114935 (2020).

[27] T. Nomura, H. Okada, and S. Patra, An inverse seesaw model with $A_{4}$-modular symmetry, arXiv:1912.00379.

[28] X. Wang, Lepton flavor mixing and $C P$ violation in the minimal type-(I + II) seesaw model with a modular $A_{4}$ symmetry, Nucl. Phys. B957, 115105 (2020).

[29] T. Kobayashi, T. Nomura, and T. Shimomura, Type II seesaw models with modular $A_{4}$ symmetry, Phys. Rev. D 102, 035019 (2020).

[30] S. J. King and S. F. King, Fermion mass hierarchies from modular symmetry, J. High Energy Phys. 09 (2020) 043.

[31] H. Okada and M. Tanimoto, Quark and lepton flavors with common modulus $\tau$ in $A_{4}$ modular symmetry, arXiv:2005 .00775 .

[32] T. Nomura and H. Okada, A linear seesaw model with $A_{4}$ modular flavor and local $U(1)_{B-L}$ symmetries, arXiv:2007 .04801 .

[33] J. Penedo and S. Petcov, Lepton masses and mixing from modular $S_{4}$ symmetry, Nucl. Phys. B939, 292 (2019).

[34] P. Novichkov, J. Penedo, S. Petcov, and A. Titov, Modular $\mathrm{S}_{4}$ models of lepton masses and mixing, J. High Energy Phys. 04 (2019) 005.

[35] I. de Medeiros Varzielas, S.F. King, and Y.-L. Zhou, Multiple modular symmetries as the origin of flavor, Phys. Rev. D 101, 055033 (2020).

[36] T. Kobayashi, Y. Shimizu, K. Takagi, M. Tanimoto, and T. H. Tatsuishi, New $A_{4}$ lepton flavor model from $S_{4}$ modular symmetry, J. High Energy Phys. 02 (2020) 097.

[37] S. F. King and Y.-L. Zhou, Trimaximal $\mathrm{TM}_{1}$ mixing with two modular $S_{4}$ groups, Phys. Rev. D 101, 015001 (2020).

[38] J. C. Criado, F. Feruglio, and S. J. King, Modular invariant models of lepton masses at levels 4 and 5, J. High Energy Phys. 02 (2020) 001.
[39] X. Wang and S. Zhou, The minimal seesaw model with a modular $\mathrm{S}_{4}$ symmetry, J. High Energy Phys. 05 (2020) 017.

[40] X. Wang, A systematic study of Dirac neutrino mass models with a modular $S_{4}$ symmetry, Nucl. Phys. B962, 115247 (2021).

[41] P. Novichkov, J. Penedo, S. Petcov, and A. Titov, Modular $\mathrm{A}_{5}$ symmetry for flavour model building, J. High Energy Phys. 04 (2019) 174.

[42] G.-J. Ding, S. F. King, and X.-G. Liu, Neutrino mass and mixing with $A_{5}$ modular symmetry, Phys. Rev. D 100, 115005 (2019).

[43] G.-J. Ding, S. F. King, C.-C. Li, and Y.-L. Zhou, Modular invariant models of leptons at level 7, J. High Energy Phys. 08 (2020) 164.

[44] J.-N. Lu, X.-G. Liu, and G.-J. Ding, Modular symmetry origin of texture zeros and quark lepton unification, Phys. Rev. D 101, 115020 (2020).

[45] G.-J. Ding and F. Feruglio, Testing moduli and flavon dynamics with neutrino oscillations, J. High Energy Phys. 06 (2020) 134.

[46] P. P. Novichkov, J. T. Penedo, S. T. Petcov, and A. V. Titov, Generalised $C P$ symmetry in modular-invariant models of flavour, J. High Energy Phys. 07 (2019) 165.

[47] A. Baur, H. P. Nilles, A. Trautner, and P. K. S. Vaudrevange, Unification of flavor, $C P$, and modular symmetries, Phys. Lett. B 795, 7 (2019).

[48] B. S. Acharya, D. Bailin, A. Love, W. A. Sabra, and S. Thomas, Spontaneous breaking of $C P$ symmetry by orbifold moduli, Phys. Lett. B 357, 387 (1995); Erratum, 407, 451 (1997).

[49] T. Dent, $C P$ violation and modular symmetries, Phys. Rev. D 64, 056005 (2001).

[50] J. Giedt, $C P$ violation and moduli stabilization in heterotic models, Mod. Phys. Lett. A 17, 1465 (2002).

[51] A. Baur, H. P. Nilles, A. Trautner, and P. K. Vaudrevange, A string theory of flavor and $\mathcal{C P}$, Nucl. Phys. B947, 114737 (2019).

[52] X.-G. Liu and G.-J. Ding, Neutrino masses and mixing from double covering of finite modular groups, J. High Energy Phys. 08 (2019) 134.

[53] X.-G. Liu, C.-Y. Yao, and G.-J. Ding, Modular invariant quark and lepton models in double covering of $S_{4}$ modular group, arXiv:2006.10722.

[54] P. Novichkov, J. Penedo, and S. Petcov, Double cover of modular $S_{4}$ for flavour model building, arXiv:2006.03058.

[55] H. Cohen and F. Strömberg, Modular Forms, Vol. 179 (American Mathematical Society, Providence, 2017).

[56] F. Strömberg, Weil representations associated with finite quadratic modules, Math. Z. 275, 509 (2013).

[57] G. Shimura, Modular forms of half integral weight, in Modular Functions of One Variable I (Springer, New York, 1973), pp. 57-74.

[58] J. H. Bruinier and O. Stein, The Weil representation and Hecke operators for vector valued modular forms, Math. Z. 264, 249 (2010).

[59] The GAP Group, GAP - Groups, Algorithms, and Programming, Version 4.10.2, 2020, https://www.gap-system.org.

[60] T. Ibukiyama, Modular forms of rational weights and modular varieties, in Abhandlungen aus dem Mathematischen Seminar 
der Universität Hamburg (Springer, New York, 2000), Vol. 70, p. 315 .

[61] T. Ibukiyama, Graded rings of modular forms of rational weights, Res. Number Theory 6, 8 (2020).

[62] R. Bruggeman, Y. Choie, and N. Diamantis, Holomorphic Automorphic Forms and Cohomology, Vol. 253 (American Mathematical Society, Providence, 2018).

[63] H. Aoki et al., On Jacobi forms of real weights and indices, Osaka J. Math. 54, 569 (2017).

[64] Y. I. Manin, Modular forms of real weights and generalised dedekind symbols, Res. Math. Sci. 5, 2 (2018).

[65] G. Shimura, On modular forms of half integral weight, Ann. Math. (N. Y.) 97, 440 (1973).

[66] U. N. D. Modulformen and H. Gewichts, Ueber den Zusammenhang Zwischen Jacobiformen und Modulformen Halbganzen Gewichts (Universitt Bonn Mathematisches Institut, Bonn, 1984).

[67] D. Z. Valery Gritsenko, N.-P. Skoruppa, and D. Zagier, Theta blocks arXiv:1907.00188.

[68] F. W. Olver, D. W. Lozier, R. F. Boisvert, and C. W. Clark, NIST Handbook of Mathematical Functions Hardback and CD-ROM (Cambridge University Press, Cambridge, England, 2010).

[69] S. Ferrara, D. Lust, A. D. Shapere, and S. Theisen, Modular invariance in supersymmetric field theories, Phys. Lett. B 225, 363 (1989).
[70] S. Ferrara, D. Lust, and S. Theisen, Target space modular invariance and low-energy couplings in orbifold compactifications, Phys. Lett. B 233, 147 (1989).

[71] I. Esteban, M. Gonzalez-Garcia, A. Hernandez-Cabezudo, M. Maltoni, and T. Schwetz, Global analysis of threeflavour neutrino oscillations: Synergies and tensions in the determination of $\theta_{23}, \delta_{C P}$, and the mass ordering, J. High Energy Phys. 01 (2019) 106.

[72] M. Tanabashi et al. (Particle Data Group Collaboration), Review of particle physics, Phys. Rev. D 98, 030001 (2018).

[73] M. Aker et al. (KATRIN Collaboration), Improved Upper Limit on the Neutrino Mass from a Direct Kinematic Method by KATRIN, Phys. Rev. Lett. 123, 221802 (2019).

[74] A. Gando et al. (KamLAND-Zen Collaboration), Search for Majorana Neutrinos near the Inverted Mass Hierarchy Region with KamLAND-Zen, Phys. Rev. Lett. 117, 082503 (2016); Phys. Rev. Lett. 117, 109903(A) 2016).

[75] N. Aghanim et al. (Planck Collaboration), Planck 2018 results. VI. Cosmological parameters, Astron. Astrophys. 641, A6 (2020).

[76] M. Cvetic, A. Font, L. E. Ibanez, D. Lust, and F. Quevedo, Target space duality, supersymmetry breaking and the stability of classical string vacua, Nucl. Phys. B361, 194 (1991). 